\title{
Unification of General Relativity and Quantum Mechanics by the Principle of Central System Relativity
}

\author{
Surendra Mund \\ Department of Physics, Student, Central University of Rajasthan, Ajmer, India
}

\begin{abstract}
This particular article is devoted to a breakthrough which is waiting for 100 years of physics. In this particular article I am unifying two kind of physical structures and law's governed by them with help of Principle of Central System Relativity obtained by me in my previous article named "Generalization of various kinds of Central Systems exist in Universe and their mathematical representations" [6]. Albert Einstein tried to unify both theories which work on macroscopic and microscopic level (Quantum Mechanics and General Relativity) in his last years as Unified field theory (UFT) but failed. Basically these both theories came from his miraculous year 1905 and 1915 or from his five excellent papers [7] which changed the fundamental understandings of theoretical physics. In this article I had obtained a Universal Principle of Central System Relativity which applied on all type of Geometrical scales exist in Universe. The Principle of Central System Relativity holds in and N-Central Systems predicted by N-Time Inflationary Model of Universe (Obtained by me in my previous paper [6]). This article unified both theories on Macroscopic and Microscopic level (Respectively General Relativity and Quantum Mechanics) into one single theory- "Universal Mechanics". So, in this particular article I have unified both Macroscopic and Microscopic structures into one single physical structure which is more unique in universal sense and also generalized (theoretically and mathematically) almost all fundamental principles of both physical structures in universal sense and then unified them with help of the Principle of Central System Relativity on each type of bodies (created during each inflation) exists in Universe. On the other hand I also obtained a very elegant principle of relativity defined by me as "Principle of Universal Relativity" which satisfies both Galilean Relativity [22] and Einstein's Relativity [7] and also includes a very different new term which appears in universal sense or not for observers on same body (like we are on Earth).
\end{abstract}

Key Words: Central System Force, Field Equations of Gravity, Flow Generated Force, Generalized Quantum Mechanics, Principle of Central System Relativity, Universal Mechanics, Universal Principle of Relativity, Variational Force.

\section{INTRODUCTION}

In a particular paper by Albert Einstein [7] on the proof of photo electric effect by the quanta of light previously predicted by Max Planck in his 1901 paper [8] was proven by Professor Leonard's photo electric effect [9]. This was the beginning of Quantum Mechanics. Later Niels Henrik Bohr came up with his famous Principle of correspondence [10] or used plank constant to describe the stability of atom in his second hypothesis [11] that angular momentum of atomic electrons in an orbit can be defined as $L=m v r=n \hbar$. In same phase Albert Einstein was totally intended towards macroscopic scale and came up with his remarkable theory of General Relativity in 1915 [12]. From here these two theories emerged with different seeming laws on macroscopic or microscopic scales. After that Louise Victor De Broglie [13] came up with his outstanding phenomenology and proved duality of nature or also proved Bohr's second hypothesis right by his $\lambda=\frac{h}{p}$. He used Albert Einstein's two famous equations from his 1905 papers [7] $E=h v$ and $E=m c^{2}$. I will prove these all theories and equations by my previous theoretical perspectives. Later Werner Heisenberg [14] came up with his remarkable Uncertainty Principle and this was not favoured by Albert Einstein because of not understanding my Principle of "Central System Relativity". As I described in my $6^{\text {th }}$ paper [6] that if we see microscopic scale, then we can't define macroscopic scale laws at position O' In universal frame of reference and vice versa. So, Einstein and Heisenberg were looking at microscopic laws and microscopic laws respectively as observer O' In terms of my Principle of Central System Relativity or could not justify to each other. This is the absolute reason behind emergence of both theories differently. Later by using his Uncertainty Principle Heisenberg and using Max Born's probability relation [15] Max Born, Klein-Jordan end Heisenberg came up with Matrix Mechanics [16] and that was the pretty beginning of the non relativistic Quantum Mechanics. After three years in 1927 Irvin Schrödinger came up with his wave mechanics and derived famous Schrödinger's wave equation by using previous Hamilton Jacobi equation [17]. This was an outstanding equation came up with Irvin Schrödinger. Later using Einstein's dispersion relation $E^{2}=p^{2} c^{2}+m_{0}^{2} c^{4}$ Schrödinger [18] intend to derive this quantum mechanically but he thought he was wrong with the negative energies and revoked his work. But later Klein and Klein-Jordan used [19] this equation to describe bosons (integral spin particles) but what about fermions (Half integral particles)? The solution of this question was obtained by Paul Edmund Dirac [20] in 1928 with his famous Dirac equation $\left(\gamma^{\mu} \partial_{\mu}-m\right) \psi=$ 0 and this was pretty beginning of relativistic Quantum Mechanics. So, this all started with into separated theories by one mistake or that particular mistake will be improved by this whole article. So, in this particular article I am providing a solution to unify these both ideologies by unique Principle of "Central System Relativity". I will also provide generalized Correspondence Principle and generalized Uncertainty Principle in sense of my ideology. I will start from the proofs of special theory of relativity and by improving the mistakes into this particular theory in my theoretical perspective, and then I am to define my Principle of equivalence, and after that I will start proving Niels Bohr classical quantum relations with help of fundamental quantities defined by me in my $5^{\text {th }}$ Article [5] like $\phi, \psi, G^{\circ}, H^{\circ}, S, Q_{c}^{\circ}$ etc. I will also prove that Wave-Particle Duality is a very classical example of $\phi-\psi$ transformation and how Uncertainty came into order at lower scales in N-time Inflationary Model of Universe. In this particular article I intend to justify that both theories are same part of a particular theory in universal frame of reference described by me in my previous articles [1-6]. In last phase of article I will unify the outcomes of my whole expressions of special and general theory of relativity or Quantum Mechanics relativistic or non-relativistic and I will prove that both theories are two faces of same coin. I will also obtain kinetic and 
potential energy relations from $E=\phi \psi$ and relation between the velocity and kinetic energy or Converged Energy as well.

\section{Explanation of Theory of Relativity and}

Fundamental Quantum Mechanics in my

\section{Theoretical Perspective}

\subsection{Explanation of Special Theory of Relativity in My Theoretical Perspective}

I have defined that speed of light is not a universal constant or speed of light varies by variation into the density of a scalar field in which it is propagating. Now let's suppose end Michelson-Morley experiment [35] like this-

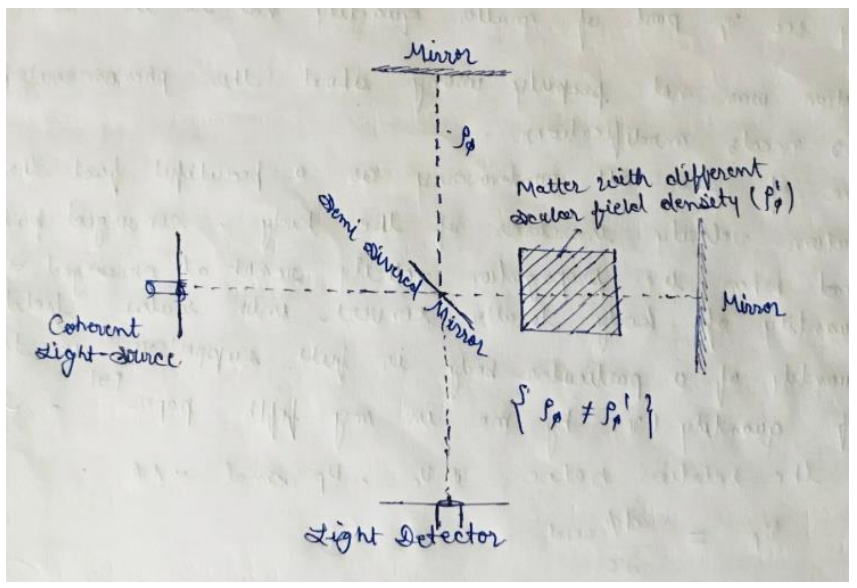

FIG. 1 Michelson-Morley Experiment with variation in scalar field density

In this case what will happen? So, in this case we will see diffraction in slit due to change in velocity of photon's or broken part in another matter. Now this idea completely declines the outcomes of special relativity but this does not completely. According to special relativity anything other than broken part (photon) can't reach to the speed of light but why in Cherenkov radiation effect [27] propagation speed of electrons exceeds the speed of light? The answer of these controversies lies in my first paper [1]. Now as I described in my first article at speed of light quantity of body does not tend to Infinity but it totally converge into scalar field. So, the relation of special relativity [7] needs some modifications -

$$
m=\frac{m_{0}}{\sqrt{1-\frac{v^{2}}{c^{2}}}}
$$

$$
\text { At } v=c, m=\infty
$$

But according to me-

$$
\psi=\psi_{p}+\alpha \Delta \phi
$$

Or

$$
\psi_{t}=\psi+\alpha \phi=\psi+\psi^{\prime}
$$

At $v=c, \psi=\alpha \phi^{\prime}$ or quantity totally converts into scalar field at this approach. As we know in different scalar field densities this velocity is different and we can say it as conversion velocity $\left(v_{c}\right)$. So, equation (1) becomes-

$$
m=\frac{m_{0}}{\sqrt{1-\frac{v^{2}}{v_{c}^{2}}}}
$$

But actually this is in terms of special relativity only. But in my sense at $v_{c^{-}}$

$$
\psi=\alpha \phi^{\prime}=\psi_{p}+\alpha \Delta \phi
$$

We can also define length contraction as- "if the quantity converts into scalar field totally at $v_{c}$, then we can't see it actually". Because we only see $\psi_{p}$ part of matter quantity not to the scalar field.

So, Albert Einstein was not properly wrong about this phenomenology, but his equations needs modifications. So, the outcome of this all controversy as beautiful fact that as the propagation velocity increases of the body, converged part also increases and when the propagation velocity reach at converged velocity, then quantity of body totally converts into scalar field. So, at quantity of a particular body is fully supportive in terms of definition of quantity $(\psi)$ by me in my fifth paper [5]. So, we need to define the relation between $v_{c}, v_{p}$ and $\alpha \Delta \phi$.

$$
\because v_{p}=\phi_{\text {covered }}=\frac{d s}{d \tau}
$$

$$
\left(v_{p}\right)_{b} \propto \varepsilon\left(\Phi_{u}\right)
$$

$$
\left(v_{p}\right)_{b} \propto \Phi_{u}
$$

These three relations were obtained in my first [1] and $5^{\text {th }}$ articles [5]. Now by adding the flow of scalar field we can write equation (6) as-

$$
\phi_{\text {covered }}=\vec{v}_{p}+k_{\phi} \overrightarrow{\mathrm{F}}\left(\Phi^{\prime}\right)+k_{\phi} \overrightarrow{\mathrm{F}}\left(\Phi_{u}\right)
$$


As we know that is a space parameter. So, we can represent it also as-

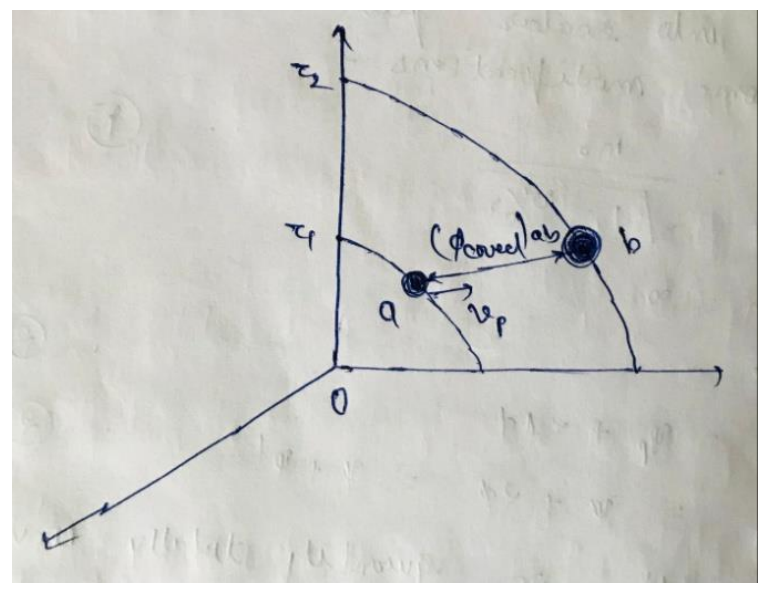

FIG. 2 Geometrical Representation of Universal Relativity

We can also write $\left(\phi_{\text {covered }}\right)_{a b}=\Delta \phi_{\text {covered }}$ between these points a $\&$ b. So, scalar field converged by a body with propagation speed $v_{p}$ and affected the motion of body flows of scalar field of body and Universe can be right as space parameter-

$$
\frac{d \vec{s}}{d \tau}=\vec{v}_{p}+k_{\phi} \overrightarrow{\mathrm{F}}\left(\Phi^{\prime}\right)+k_{\phi} \overrightarrow{\mathrm{F}}\left(\Phi_{u}\right)=\phi_{\text {covered }}
$$

Here $k_{\phi}$ is some parameter. Here space parameter for simplification. Now the quantity of motion can be written as-

$$
\vec{F}=\psi \frac{d \vec{s}}{d \tau}=\left(\psi_{p}+\alpha \Delta \phi\right) \frac{d \vec{s}}{d \tau}
$$

Now by putting equation (10) in equation (11), we get-

$$
\begin{array}{r}
\vec{F}=\left(\psi_{p}+\alpha \Delta \phi\right) \vec{v}_{p}+\left(\psi_{p}+\alpha \Delta \phi\right) k_{\phi} \overrightarrow{\mathrm{F}}\left(\Phi^{\prime}\right) \\
+k_{\phi^{\prime}}\left(\psi_{p}+\alpha \Delta \phi\right) \overrightarrow{\mathrm{F}}\left(\Phi_{u}\right)
\end{array}
$$

Here third part includes the universal motion and in first term is the actual propagation velocity of body. For simplicity we can write equation (12) as-

$$
\begin{aligned}
\left(\psi_{p}+\alpha \Delta \phi\right)\left(v_{p}\right)_{\text {apperent }} & \\
& =\left(\psi_{p}+\alpha \Delta \phi\right)\left(v_{p}\right)_{\text {actual }} \\
& +\left(\psi_{p}+\alpha \Delta \phi\right) k_{\phi} \mathrm{F}\left(\Phi^{\prime}\right) \\
& +k_{\phi \prime}\left(\psi_{p}+\alpha \Delta \phi\right) \mathrm{F}\left(\Phi_{u}\right)
\end{aligned}
$$

$$
\left(v_{p}\right)_{\text {apperent }}=\frac{d s}{d \tau}
$$

From here an outstanding thing comes out that let's assume two observers $\mathrm{O}_{1}$ or $\mathrm{O}_{2}$ respectively on bodies $\mathrm{B}_{1}$ or $\mathrm{B}_{2}$ moving with some actual velocities $v_{1}$ and $v_{2}$. Here both bodies have different conversion constants and different scalar field flows and different universal motions, then the relative speed of observer one with respect to observer two will be-

$$
\left(\vec{v}_{2}\right)_{\text {apperent }}-\left(\vec{v}_{1}\right)_{\text {apperent }}=\left(\vec{v}_{12}\right)_{\text {apperent }}
$$

Now by using equation (13) and (14), we get-

$$
\left(\vec{v}_{2}\right)_{\text {apperent }}=\vec{v}_{2}+k_{\phi} \overrightarrow{\mathrm{F}}\left(\Phi_{B_{2}}\right)+k_{\phi} \overrightarrow{\mathrm{F}}_{B_{2}}\left(\Phi_{u}\right)
$$

$$
\left(\vec{v}_{1}\right)_{\text {apperent }}=\vec{v}_{1}+k_{\phi} \overrightarrow{\mathrm{F}}\left(\Phi_{B_{1}}\right)+k_{\phi} \overrightarrow{\mathrm{F}}_{B_{1}}\left(\Phi_{u}\right)
$$

By using equations (16) and (17) in equation (15), we get relative velocity-

$$
\begin{aligned}
\left(\vec{v}_{12}\right)_{\text {apperent }}=( & \left.\vec{v}_{2}-\vec{v}_{1}\right)+k_{\phi}\left\{\overrightarrow{\mathrm{F}}\left(\Phi_{B_{2}}\right)-\overrightarrow{\mathrm{F}}\left(\Phi_{B_{1}}\right)\right\} \\
& +k_{\phi},\left\{\overrightarrow{\mathrm{F}}_{B_{2}}\left(\Phi_{u}\right)-\overrightarrow{\mathrm{F}}_{B_{1}}\left(\Phi_{u}\right)\right\}
\end{aligned}
$$

Here according to Galileo's Principle of relativity [22] the first term is valid and according to Einstein's Principle of relativity [7] the first and second terms is valid but what about the third term? So, from here "Universal Principle of Relativity" comes out by the $3^{\text {rd }}$ term including in equation (18). This universal Principle of relativity holds in my formerly described universal frame of reference. In Principle of Universal Relativity Ernst Mach's Principle (23) is also satisfied. If expansion of Universe is correct or Universe is in motion (proved by Edwin Hubble [24]) then, Principle of Universal Relativity is correct. We can use simplified symbols for apparent velocity as $\vec{v}_{p}^{\prime}$ or actual velocity $\vec{v}_{p}$. So, $\vec{v}_{p}^{\prime}$ can be written as-

$$
\vec{v}_{p}^{\prime}=\vec{v}_{p}+k_{\phi} \overrightarrow{\mathrm{F}}\left(\Phi_{B}\right)+k_{\phi} \overrightarrow{\mathrm{F}}\left(\Phi_{u}\right)
$$

Now from my former paper [6]-

$$
d \tau=\frac{1}{\Phi \Gamma(\Phi)} . d \Phi
$$


So, we know from equation (14)-

$$
d \vec{s}=\vec{v}_{p}^{\prime} \cdot d \tau
$$

Now by putting value of from equation (19) in equation (21), we get-

$$
d s=\left\{v_{p}+k_{\phi} \mathrm{F}\left(\Phi_{B}\right)+k_{\phi} \mathrm{F}_{B}\left(\Phi_{u}\right)\right\} \frac{d \Phi_{B}}{\Phi_{B} \Gamma\left(\Phi_{B}\right)}
$$

Here $\Phi_{B}$ is the scalar field around the body. We can write equation

$$
v_{p}+k_{\phi} \mathrm{F}\left(\Phi_{B}\right)+k_{\phi} \mathrm{F}_{B}\left(\Phi_{u}\right)=\Phi_{B} \Gamma\left(\Phi_{B}\right) \frac{d s}{d \Phi_{B}}
$$

Now I come to a beautiful relationship from the definition I proposed in my previous Article [5] for quantity of motion or the supported part $(\alpha \Delta \phi)$ or opposed part $\left(\psi_{p}\right)$. So, motion can be explained as-

$$
\begin{gathered}
\psi \frac{d s}{d \tau} \propto \alpha \Delta \phi \\
\psi \frac{d s}{d \tau} \propto \frac{1}{\psi_{p}}
\end{gathered}
$$

So, actual and apparent both propagation speeds also specified from relations (24) and (25) as-

$$
\begin{gathered}
v_{p} \propto \alpha \Delta \phi \\
v_{p} \propto \frac{1}{\psi_{p}}
\end{gathered}
$$

Now by conservation of total quantity of body, we can write for a body as-

$$
\psi_{t}=\psi+\psi^{\prime}=\psi_{p}+\alpha \Delta \phi+\psi^{\prime}=\text { constant }
$$

Or variation in total quantity of a body will be zero as-

$$
\Delta \psi_{t}=0
$$

Now by taking variation of equation (28), we get-

$$
\Delta \psi_{p}+\Delta \alpha \Delta \phi+\alpha \Delta^{2} \phi+\Delta \alpha \phi+\alpha \Delta \phi=0
$$

Now by putting similar terms together, we get-

$$
\Delta \psi_{p}+\alpha\left(\Delta \phi+\Delta^{2} \phi\right)+\Delta \alpha(\phi+\Delta \phi)=0
$$

Now by putting some proportionality constants in equation (26) and (27), we get-

$$
v_{p}=\xi_{c} \alpha \Delta \phi
$$

$$
v_{p}=\frac{\xi_{p}}{\psi_{p}}
$$

These both relations are the keyhole to understand the fundamental things in Central System Relativity. Now by putting value in equation (21), we get-

$$
d s=v_{p}^{\prime} \frac{d \Phi_{B}}{\Phi_{B} \Gamma\left(\Phi_{B}\right)}
$$

Now we can write above equation as-

$$
\text { ds. } \Phi_{B} \Gamma\left(\Phi_{B}\right)=v_{p}^{\prime} d \Phi_{B}
$$

Now by taking variation both sides in equation (35), we get-

$$
\begin{aligned}
\Delta(\Delta s) . \Phi_{B} \Gamma\left(\Phi_{B}\right) & +\Delta s . \Delta \Phi_{B} \Gamma\left(\Phi_{B}\right)+\Delta s . \Phi_{B} \Delta \Gamma\left(\Phi_{B}\right) \\
& =\Delta v_{p}^{\prime} \Delta \Phi_{B}+v_{p}^{\prime} \Delta\left(\Delta \Phi_{B}\right)
\end{aligned}
$$

By some manipulations we can write equation (36), as-

$$
\begin{gathered}
\Delta v_{p}^{\prime}=\frac{\Phi_{B}}{\Delta \Phi_{B}}\left\{\Delta^{2} s .\right. \\
-v_{p}^{\prime} \frac{\Delta\left(\Delta \Phi_{B}\right)}{\Delta \Phi_{B}}
\end{gathered}
$$

Now we can also write equation (34) as-

$$
v_{p}^{\prime}=\frac{\Phi_{B}}{\Delta \Phi_{B}}\left(\Delta s . \Gamma\left(\Phi_{B}\right)\right)
$$


Now by putting relation (38) in (37), we get-

$$
\begin{gathered}
\Delta v_{p}^{\prime}=\frac{\Phi_{B}}{\Delta \Phi_{B}}\left\{\Delta^{2} s . \Gamma\left(\Phi_{B}\right)+\Delta s . \Delta \Gamma\left(\Phi_{B}\right)\right\}++\Delta s . \Gamma\left(\Phi_{B}\right) \\
-\frac{\Phi_{B}}{\Delta \Phi_{B}} \frac{\Delta s . \Gamma\left(\Phi_{B}\right) \Delta^{2} \Phi_{B}}{\Delta \Phi_{B}}
\end{gathered}
$$

Now by putting similar terms common in equation (39), we get-

$$
\begin{aligned}
\Delta v_{p}^{\prime}=\frac{\Phi_{B}}{\Delta \Phi_{B}}\left\{\Delta^{2} s .\right. & \Gamma\left(\Phi_{B}\right)+\Delta s . \Delta \Gamma\left(\Phi_{B}\right) \\
& \left.+\Delta s . \Gamma\left(\Phi_{B}\right)\left(\frac{\Delta \Phi_{B}}{\Phi_{B}}-\frac{\Delta^{2} \Phi_{B}}{\Delta \Phi_{B}}\right)\right\}
\end{aligned}
$$

$$
\left\{\operatorname{Here} \Gamma\left(\Phi_{B}\right)=\operatorname{Tr}\left(\Gamma_{\mu \nu . . n}\left(\Phi_{B}\right)\right)\right\}
$$

We can also write equation (40) as-

$$
\begin{aligned}
\Delta v_{p}^{\prime}=\frac{\Phi_{B}}{\Delta \Phi_{B}}\left\{\Delta^{2} s .\right. & \Gamma\left(\Phi_{B}\right) \\
& \left.+\Delta s\left[\Delta \Gamma\left(\Phi_{B}\right)+\Gamma\left(\Phi_{B}\right)\left(\frac{\Delta \Phi_{B}}{\Phi_{B}}-\frac{\Delta^{2} \Phi_{B}}{\Delta \Phi_{B}}\right)\right]\right\}
\end{aligned}
$$

From above equation, we can write-

$$
\begin{aligned}
\Delta \Phi_{B} \Delta v_{p}^{\prime}=\Phi_{B}[ & \Delta s . \Delta \Gamma\left(\Phi_{B}\right) \\
& \left.+\Gamma\left(\Phi_{B}\right)\left\{\Delta^{2} s+\Delta s\left(\frac{\Delta \Phi_{B}}{\Phi_{B}}-\frac{\Delta^{2} \Phi_{B}}{\Delta \Phi_{B}}\right)\right\}\right]
\end{aligned}
$$

Here two terms comes out like Uncertainty relation-

$$
\left\{\begin{array}{c}
\Delta \Phi_{B} \cdot \Delta v_{p}^{\prime} \\
\Delta s . \Delta \Gamma\left(\Phi_{B}\right)
\end{array}\right.
$$

Now I am defining a "Generalized Uncertainty Principle" according to Principle of Central System Relativity. At first I am representing this thing in universal frame of reference. As I described formerly in introduction that Uncertainty relation and Wave-Particle Duality both are beautiful manifestations of $\phi-\psi$ transformation. So, by the below representation-

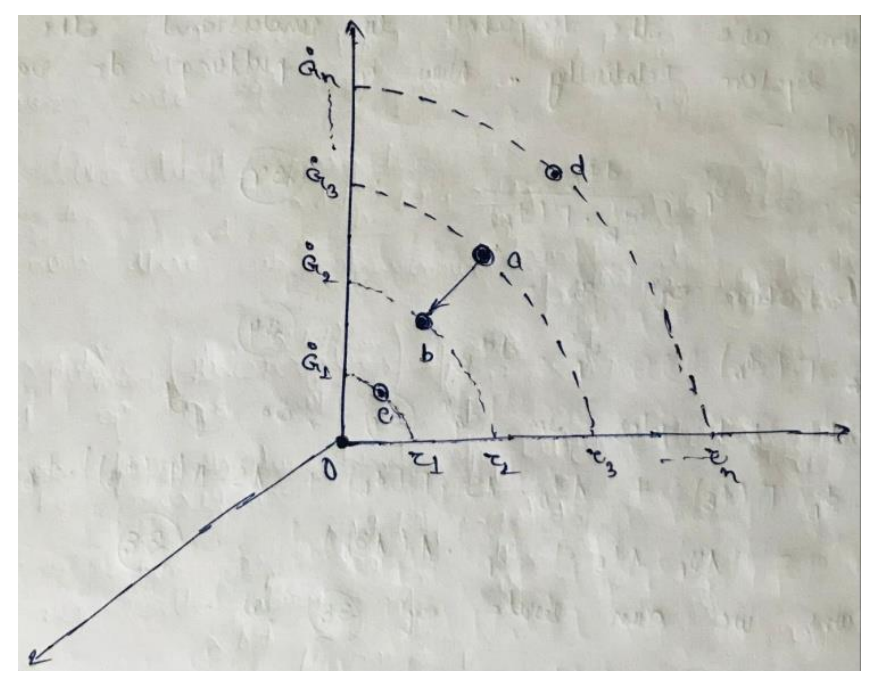

FIG. 3 Geometrical Representation of Generalized Uncertainty Relation

In this representation if observer at position $a$ is determining laws of physics on lower geometries ' $b$ ' by using the broken parts of lower bodies on certain quantity of motion and as we know quantity of motion transforms some quantity of body in scalar field which is $\phi-\psi$ transformation over we cannot see the transformed quantity by broken parts than we observer a will never specify the certain position of body. This thing can be written also as mathematically-

For Heisenberg Uncertainty the same thing happen here observer ' $a$ ' from solar systems is determining the laws of physics on lower geometrical scale atomic Central Systems 'b'. So, Uncertainty arises due to $\phi-\psi$ transformation or we can write it as a product of this $\phi-\psi$ transformation-

\section{$\Delta \phi . \Delta \psi$}

From here a Generalized Uncertainty Principle comes out that "If an observer from geometry $G_{i}^{\circ}$ is determining the physical laws in shorter geometries $G_{i-1}^{\circ}$ by using the broken parts of shorter geometries, then due to $\phi-\psi$ transformation in these geometries he will never observe the fundamental physical quantities (like Quantity of Body $(\psi)$, Scalar Field $(\phi)$, time, position, Quantity of Motion etc) precisely with respect to each other". This low holds only in $a \rightarrow b$ conditions. Now what happens when observer ' $\mathrm{a}$ ' is looking at $G_{1}^{\circ}$ or ' $c$ '? In this case the observer will never determine the existence of $G_{1}^{\circ}$ geometries by using the broken parts of ' $b$ ' because these broken parts are also formed out by ' $c$ ' geometries.

As we know waves are properties of medium. So, by above $\phi$ $\psi$ transformation "when quantity converts in a scalar field it seems like waves (uniform property) but when this converted 
quantity of scalar field again converts in scalar field it behaves like particle (discrete property) and this is the wave-particle duality in sense of $\phi-\psi$ transformation".

As we know that de Broglie wavelength relation [13]-

$$
\lambda=\frac{h}{p}
$$

In my theoretical perspective

$$
\begin{gathered}
\lambda=\frac{h}{F} \\
\because h=2 \pi \cdot \hbar=2 \pi s^{\prime} \frac{\psi_{p}}{\alpha \Delta \phi} \\
\left\{\text { here } s^{\prime}=\frac{s}{n}\right\}
\end{gathered}
$$

Now by putting the values of $F$ from equation (11) and from equation (47), we get equation (46) as-

$$
\lambda=\frac{2 \pi s^{\prime} \psi_{p} . d \tau}{\alpha \Delta \phi\left(\psi_{p}+\alpha \Delta \phi\right) d s}
$$

We can also write above equation as-

$$
\lambda=\frac{2 \pi s^{\prime} \psi_{p}}{\alpha \Delta \phi\left(\psi_{p}+\alpha \Delta \phi\right) v_{p}^{\prime}}
$$

Now by some basic manipulations, we get-

$$
\frac{2 \pi}{\lambda}=\frac{\alpha \Delta \phi\left(1+\frac{\alpha \Delta \phi}{\psi_{p}}\right) \cdot v_{p}^{\prime}}{\mathcal{s}^{\prime}}
$$

As we know-

$$
\begin{gathered}
v \lambda=\left(v_{p}\right)_{c} \\
\left\{\frac{2 \pi}{\lambda}=k=\text { wave number }\right\}
\end{gathered}
$$

So, we can write equation (50) as-

$$
k=\frac{\alpha \Delta \phi}{s^{\prime}} \cdot\left(1+\frac{\alpha \Delta \phi}{\psi_{p}}\right) \cdot v_{p}^{\prime}
$$

Now by using equation (51), we get equation (50) as-

$$
\frac{2 \pi \nu}{\left(v_{p}\right)_{c}}=\frac{\alpha \Delta \phi}{s^{\prime}} \cdot\left(1+\frac{\alpha \Delta \phi}{\psi_{p}}\right) \cdot v_{p}^{\prime}
$$

$$
\because 2 \pi v=\omega=\text { angular frequency }
$$

Now by putting the values of $2 \pi \nu$ in equation (53), we get-

$$
\frac{\omega}{\left(v_{p}\right)_{c}}=\frac{\alpha \Delta \phi}{s^{\prime}} \cdot\left(1+\frac{\alpha \Delta \phi}{\psi_{p}}\right) \cdot v_{p}^{\prime}
$$

Now by putting the value of $v_{p}^{\prime}$ from equation (19), we get equation (55) as-

$$
\frac{\omega}{\left(v_{p}\right)_{c}}=\frac{\alpha \Delta \phi}{s^{\prime}} \cdot\left(1+\frac{\alpha \Delta \phi}{\psi_{p}}\right) \cdot\left(\vec{v}_{p}+k_{\phi} \overrightarrow{\mathrm{F}}\left(\Phi_{B}\right)+k_{\phi^{\prime}} \overrightarrow{\mathrm{F}}\left(\Phi_{u}\right)\right)
$$

Now by some manipulations, we get-

$$
\frac{\left(v_{p}\right)_{c}}{\omega}\left(\vec{v}_{p}+k_{\phi} \overrightarrow{\mathrm{F}}\left(\Phi_{B}\right)+k_{\phi^{\prime}} \overrightarrow{\mathrm{F}}\left(\Phi_{u}\right)\right)=\frac{s^{\prime}}{\alpha \Delta \phi} \frac{1}{\left(1+\frac{\alpha \Delta \phi}{\psi_{p}}\right)}
$$

Here two conditions comes out-

1. Condition-1 when body is reaching the critical velocity, then-

$$
v_{p} \approx\left(v_{p}\right)_{c} \gg k_{\phi} \overrightarrow{\mathrm{F}}\left(\Phi_{B}\right)+k_{\phi^{\prime}} \overrightarrow{\mathrm{F}}\left(\Phi_{u}\right)
$$

$$
\frac{\left(v_{p}\right)_{c}^{2}}{\omega}=\frac{s^{\prime}}{\alpha \Delta \phi} \frac{1}{\left(1+\frac{\alpha \Delta \phi}{\psi_{p}}\right)}
$$

So, we can write equation (57) as-

$$
(\alpha \Delta \phi)_{\max }\left(1+\frac{(\alpha \Delta \phi)_{\max }}{\psi_{p}}\right)=\frac{s^{\prime} \omega_{c}}{\left(v_{p}\right)_{c}^{2}}
$$


(60)

As we know there is maximum five side $\phi-\psi$ transformation in a body at critical velocity.

As we know for similar kind of bodies critical speed is constant in same scalar field density. So, spin of a body will be at maximum critical speed.

2. Condition-2 when $v_{p}^{\prime} \rightarrow 0$, then$\alpha \Delta \phi \ll \psi_{p}$

Or

$$
\frac{\alpha \Delta \phi}{\psi_{p}} \ll 1
$$

So, we can write equation (57) as-

$$
\frac{v_{p}^{\prime}\left(v_{p}\right)_{c}}{\omega}=\frac{s^{\prime}}{\alpha \Delta \phi}
$$

We can calculate $\left(v_{p}\right)_{c}$ (critical velocity) of a particular classical body as-

$$
\left(v_{p}\right)_{c}=\frac{s^{\prime} \omega}{\alpha \Delta \phi \cdot v_{p}^{\prime}}
$$

Now by using relations (32) and (33) in equation (57), we get-

$$
\begin{aligned}
& \frac{v_{p}^{\prime}\left(v_{p}\right)_{c}}{\omega}=\frac{s^{\prime} \cdot \xi_{c}}{v_{p}\left(1+\frac{v_{p} \cdot v_{p}}{\xi_{c} \xi_{p}}\right)} \\
& \left\{\because \psi_{p}=\frac{\xi_{p}}{v_{p}}, \alpha \Delta \phi=\frac{v_{p}}{\xi_{c}}\right\}
\end{aligned}
$$

Now by putting velocity parts together, we get-

$$
\frac{v_{p}^{\prime} \cdot v_{p} \cdot\left(v_{p}\right)_{c}}{\omega}=\frac{s^{\prime} \cdot \xi_{c}}{\left(1+\frac{v_{p}^{2}}{\xi_{c} \xi_{p}}\right)}
$$

$$
\frac{\left(v_{p}\right)_{c}^{3}}{\omega_{c}}=\frac{s^{\prime} \cdot \xi_{c}}{\left(1+\frac{\left(v_{p}\right)_{c}^{2}}{\xi_{c} \xi_{p}}\right)}
$$

So, $\omega_{c}$ will be at critical velocity-

$$
\omega_{c}=\frac{\left(v_{p}\right)_{c}^{3}}{s^{\prime} \cdot \xi_{c}} \cdot\left(1+\frac{\left(v_{p}\right)_{c}^{2}}{\xi_{c} \xi_{p}}\right)
$$

We can also calculate remained quantity $\psi_{p}$ at critical velocity in body as-

$$
\because \psi=\psi_{p}+\alpha \Delta \phi=\text { constant with velocity increase }
$$

$$
\left(\psi_{p}+\alpha \Delta \phi\right)=\psi_{v_{p}=0}=\psi_{v_{p}=\left(v_{p}\right)_{c}}=\left(\psi_{p}\right)_{c}+(\alpha \Delta \phi)_{\max }
$$

Now from equation (60), the critical quantity-

$$
\left(\psi_{p}\right)_{c}=\frac{(\alpha \Delta \phi)_{\max }^{2}\left(v_{p}\right)_{c}^{2}}{s^{\prime} \cdot \omega_{c}-\left(v_{p}\right)_{c}^{2} \cdot(\alpha \Delta \phi)_{\max }}
$$

So, something remains in body as perfect quantity at critical speed also, but in broken parts this quantity is totally convertible. So, in case of Cherenkov radiation effect the electron exceeds the velocity of light because its Perfect quantity not becomes zero at speed of light. Now by multiplying both relations (32) and (33), we get-

$$
v_{p}^{2}=\xi_{c} \xi_{p} \cdot \frac{\alpha \Delta \phi}{\psi_{p}}
$$

From my $5^{\text {th }}[5]$ article-

$$
S=s \frac{\psi_{p}}{\alpha \Delta \phi}
$$

Now by putting (72) in equation (71), we get-

$$
v_{p}^{2} \cdot \frac{S}{s}=\xi_{c} \xi_{p}
$$

At critical velocity-

We can also write above equation as- 


$$
v_{p}^{2}=\frac{\xi_{c} \xi_{p} \mathcal{S}}{S}
$$

Now by some other manipulations and multiplying (32) and (33), we get-

$$
\psi_{p} . \alpha \Delta \phi=\frac{\xi_{p}}{\xi_{c}}
$$

Now by putting value of $\xi_{p}$ from equation (75) in equation (74), we get-

$$
\begin{gathered}
v_{p}^{2}=\psi_{p} \cdot \alpha \Delta \phi \frac{8 \cdot \xi_{c}^{2}}{S} \\
v_{p}^{2}=(\alpha \Delta \phi)^{2} \cdot \xi_{c}^{2}
\end{gathered}
$$

Now as we know equation (75) is energy term-

$$
E_{\xi}=\psi_{p} . \alpha \Delta \phi=\frac{\xi_{p}}{\xi_{c}}
$$

Now by putting-

$$
\frac{\psi_{p}}{\alpha \Delta \phi}=\frac{1}{\gamma}
$$

Or

$$
\frac{\alpha \Delta \phi}{\psi_{p}}=\gamma
$$

By using relation (79), we can write equation (71) as-

$$
v_{p}^{2}=\xi_{c} \xi_{p} \cdot \gamma
$$

Now the relation between $E_{\xi}$ and $\gamma$ becomes-

$$
v_{p}^{2}=\gamma E_{\xi} \cdot \xi_{c}^{2}
$$

Or

$$
E_{\xi}=\frac{1}{\gamma \xi_{p}^{2}} v_{p}^{2}
$$

The energy is similar to the kinetic energy in body-

$$
E_{k}=\frac{1}{2} \psi v_{p}^{2}
$$

If $E_{k}=n \alpha E_{\xi}$, then-

$$
\psi=\frac{2 n \alpha}{\gamma \xi_{p}^{2}}=\frac{2 n \alpha \psi_{p}(\alpha \Delta \phi)^{2}}{\alpha \Delta \phi \cdot v_{p}^{2}}
$$

Here $\mathrm{n}$ is some number.

$$
\psi=\frac{2 n \alpha \psi_{p} \cdot \alpha \Delta \phi}{v_{p}^{2}}
$$

$$
v_{p}^{2}=\frac{2 n \alpha \psi_{p} \cdot \alpha \Delta \phi}{\psi}=\frac{2 n \alpha \psi_{p} \cdot \alpha \Delta \phi}{\psi_{p}+\alpha \Delta \phi}
$$

From equation (87), we get-

$$
\frac{1}{v_{p}^{2}}=\frac{1}{2 n \alpha}\left(\frac{1}{\psi_{p}}+\frac{1}{\alpha \Delta \phi}\right)
$$

We can also write above equation as-

$$
\frac{2 n \alpha}{v_{p}^{2}}=\left(\frac{1}{\psi_{p}}+\frac{1}{\alpha \Delta \phi}\right)
$$

$$
\frac{1}{\left(\frac{v_{p}}{\sqrt{2 n \alpha}}\right)}=\frac{1}{\psi_{p}}+\frac{1}{\alpha \Delta \phi}
$$

Now as we know-

$$
E=\psi \phi=\psi_{p} \phi+\alpha \phi \Delta \phi=\alpha \phi^{\prime} \Delta \phi=\frac{\psi \psi^{\prime}}{\alpha}
$$

$$
E_{\xi}=\frac{1}{\gamma}\left(\frac{v_{p}}{\xi_{p}}\right)^{2}
$$




$$
\frac{E_{\xi}}{\alpha}=\psi_{p} \Delta \phi
$$

Now by squaring-

$$
E^{2}=\left(\psi_{p} \phi\right)^{2}+(\alpha \phi \Delta \phi)^{2}+2 \psi_{p} \alpha \Delta \phi \phi^{2}
$$

$\because E_{p}=\psi_{p} \phi, E_{c}=\alpha \phi \Delta \phi$ or by putting the value of $E_{\xi}$, we get above equation as-

$$
\begin{gathered}
E^{2}=E_{p}^{2}+E_{c}^{2}+2 E_{\xi} \phi^{2} \\
E_{\phi}=\phi \phi^{\prime}=\phi^{2}\left(\frac{\phi^{\prime}}{\phi}\right)=\phi^{2}\left(\frac{\alpha \phi^{\prime}}{\alpha \phi}\right)=\phi^{2}\left(\frac{\psi^{\prime}}{\psi}\right)
\end{gathered}
$$

Or

$$
\phi^{2}=E_{\phi} \cdot\left(\frac{\alpha \phi}{\psi}\right)=\beta E_{\phi}
$$

Here $\beta$ always less than 1 by $\psi>\alpha \phi$ or positive energy theorem.

$$
\beta=\frac{\alpha \phi}{\psi}
$$

Now by putting the value of $\phi^{2}$ in equation (94), we get-

$$
E^{2}=E_{p}^{2}+E_{c}^{2}+2 \beta E_{\phi} E_{\xi}
$$

Now from here a beautiful relation come out-

$$
\beta E_{\phi} E_{\xi}=\psi_{p} \alpha \Delta \phi \phi^{2}=\left(\psi_{p} \phi\right) \cdot(\alpha \phi \Delta \phi)=E_{p} . E_{c}
$$

So, we get-

$$
E_{p} . E_{c}=\beta E_{\phi} E_{\xi}=\frac{\beta}{\alpha^{2}} E_{\psi} E_{\xi}=\frac{\beta}{\alpha} E E_{\xi}
$$

Now by dispersion relation [7]

$$
E^{2}=p^{2} c^{2}+m_{0}^{2} c^{4}
$$

If we take an approximation in my theoretical perspective, then-

$$
\left\{\begin{array}{c}
m_{0} \cong \psi_{p}(\text { non }- \\
\text { transformable quatity easily) } \\
p \equiv F(\text { Quantity of Motion }) \\
c=v_{p_{c}} \text { (Critical Velocity) }
\end{array}\right.
$$

Now by replacing symbols in equation 101, we get-

$$
E^{2}=F^{2}\left(v_{p}\right)_{c}^{2}+\psi_{p}^{2}\left(v_{p}\right)_{c}^{4}
$$

Now by putting the value of in equation (102), we get-

$$
E^{2}=\psi^{2}\left(v_{p}^{\prime}\right)^{2}\left(v_{p}\right)_{c}^{2}+\psi_{p}^{2}\left(v_{p}\right)_{c}^{4}
$$

Now by squaring equation (63), we get-

$$
\left(v_{p}^{\prime}\right)^{2}\left(v_{p}\right)_{c}^{2}=\frac{{s^{\prime}}^{2} \omega^{2}}{(\alpha \Delta \phi)^{2}} \frac{1}{(1+\gamma)^{2}}
$$

Now by squaring equation (59), we get-

$$
\left(v_{p}\right)_{c}^{4}=\frac{{\mathcal{s}^{\prime}}^{2} \omega_{c}^{2}}{(\alpha \Delta \phi)_{\max }^{2}} \frac{1}{\left(1+\gamma_{\max }\right)^{2}}
$$

Now by putting equation (105) and equation (10)4, in equation (103), we get-

$$
\begin{aligned}
E^{2}=\left(\psi_{p}+\alpha \Delta \phi\right)^{2} & \frac{{\mathcal{s}^{\prime}}^{2} \omega^{2}}{(\alpha \Delta \phi)^{2}} \frac{1}{(1+\gamma)^{2}} \\
& +\psi_{p}^{2} \frac{{s^{\prime 2}}^{\prime 2} \omega_{c}^{2}}{(\alpha \Delta \phi)_{\max }^{2}} \frac{1}{\left(1+\gamma_{\max }\right)^{2}}
\end{aligned}
$$

According to my terminology-

$$
E^{2}=\left(\psi_{p} \phi\right)^{2}+(\alpha \phi \Delta \phi)^{2}+2 \psi_{p} \alpha \Delta \phi \phi^{2}
$$

Now by putting for shake of simplicity-

$$
\epsilon^{2}=\frac{s^{\prime 2} \omega^{2}}{(\alpha \Delta \phi)^{2}} \frac{1}{(1+\gamma)^{2}} \text { or } \epsilon_{c}^{2}=\frac{s^{\prime 2} \omega_{c}^{2}}{(\alpha \Delta \phi)_{\max }^{2}} \frac{1}{\left(1+\gamma_{\max }\right)^{2}}
$$

And by solving further equation (106), we get-

$$
E^{2}=\psi_{p}^{2}\left(\epsilon^{2}+\epsilon_{c}^{2}\right)+(\epsilon \cdot \alpha \Delta \phi)^{2}+2 \psi_{p} \alpha \Delta \phi \epsilon^{2}
$$


Now if we put only $m_{0} \cong \psi$, then-

$$
E^{2}=\psi_{p}^{2}\left(\epsilon^{2}+\epsilon_{c}^{2}\right)+(\alpha \Delta \phi)^{2}\left(\epsilon^{2}+\epsilon_{c}^{2}\right)+2 \psi_{p} \alpha \Delta \phi\left(\epsilon^{2}+\epsilon_{c}^{2}\right)
$$

Now by comparing equation (107) with (108), we get-

$$
\phi^{2} \cong \epsilon^{2} \text { for } \epsilon^{2} \gg \epsilon_{c}^{2} \text { only. }
$$

$\because$ The first term-

$$
\phi^{2}=\epsilon^{2}\left(1+\left(\frac{\epsilon_{c}}{\epsilon}\right)^{2}\right)
$$

For only $\epsilon_{c} \ll \epsilon$ this term holds.

But for the approximation $m_{0} \cong \psi$, the relation becomes more unique-

$$
\phi^{2} \cong \epsilon^{2}+\epsilon_{c}^{2}
$$

So, special relativity is just a nice approximation of $\phi-\psi$ transformation, but not a unique theory in universal sense.

As we know the force exerted on a body in Central System by variation in density of scalar field as-

$$
\vec{\delta}_{\rho_{\phi}}=k \alpha_{p_{i}} \psi_{p e r}\left(\Delta \rho_{\phi}\right)_{i n}
$$

We can also write this force by taking gradient to be scalar field density as-

$$
\vec{\delta}_{\rho_{\phi}}=k \alpha_{p_{i}} \psi_{p e r} \nabla\left(\rho_{\phi_{i n}}\right)
$$

$$
\text { Here } \psi_{\text {per }}=\left(\psi_{p}+\alpha \Delta \phi\right), \because \rho_{\phi}=\frac{\Phi}{\mathcal{V}\left(\boldsymbol{M}^{\mathrm{n}}\right)}
$$

\subsection{Explanation of Principles of Quantum Mechanics in Universal Sense}

At first let's have a look on Niels Bohr Correspondence Principle [10] which played keyhole in development of Quantum Mechanics in its childhood days. This Principle is also known as classical quantum correspondence or Copenhagen interpretation. So, in starting of this discussion I am providing a particular theorem that "Each and every type of Central Systems has their own spin quantity (like $\hbar$ for atomic Central Systems). So, for solar systems we can define it as $\hbar_{s}$ for galactic Central Systems we can define it as $\hbar_{g}$ and these quantities work in my Generalized Uncertainty Principle for each and every kind of geometry exists in Universe".

Now Niels Bohr defined his Correspondence Principle as "Any quantum physical quantity should tend to its classical counterpart in the macroscopic limit. Thus, Classical Mechanics suit in Principle be recovered in that limit". There are physical quantities with no classical counterpart (example spin). But according to my N-Time Inflationary Model of Universe spin also exists in each inflation generated Central System. I also defined spin in each Central System as fundamental quantity which transforms into bigger bodies from shorter bodies in my former paper [5] as-

$$
S=s \frac{\psi_{p}}{\alpha \Delta \phi}
$$

It also can be written in form of solar systems as-

$$
n \hbar_{s}=\left(s \frac{\psi_{p}}{\alpha \Delta \phi}\right)_{\text {soler systems }}
$$

Or

$$
\hbar_{s}=s^{\prime} \frac{\psi_{p}}{\alpha \Delta \phi}
$$

Bohr's Correspondence Principle is separating Quantum Mechanics (Macroscopic Mechanics) from Classical Mechanics in terms of spin. But Niels Bohr did not understand Central System Relativity. So, I am here generalizing the Correspondence Principle as-"When if there is a change in Central System occurs, their basic properties are seems different (like spin quantity) (according to observer in Central System Relativity. So, these properties can't be described by another Central Systems (like Quantum Mechanics for atom or Classical Mechanics for solar systems) equations because they if old and created in different inflations of Universe (from N-Time Inflationary Model of Universe) but there these fundamental properties can be described by similar equations by observer at origin of universal frame of reference or in other words mechanics of each and every type of Central Systems or bodies should tend to its universal counter point with reference of observer at origin of Universe". This can be identified as "Generalized Correspondence Principle".

Now by Niels Bohr's second hypothesis [11]-

$$
m v r=n \hbar=L
$$

As we know from my $5^{\text {th }}$ paper [5]- 


$$
n \hbar=s \frac{\psi_{p}}{\alpha \Delta \phi}
$$

Now by putting $m=\psi, v=v_{p}^{\prime}, \mathrm{r}$ as $r$ and by using equation (118) in equation (117) as-

$$
\psi v_{p}^{\prime} r=s \frac{\psi_{p}}{\alpha \Delta \phi}
$$

Here $\mathrm{r}$ is the radius of body from Centre.

$$
v_{p}^{\prime}=s \frac{\psi_{p}}{\left(\psi_{p}+\alpha \Delta \phi\right) \cdot r \cdot \alpha \Delta \phi}
$$

Now by solving further, we get-

$$
v_{p}^{\prime}=\frac{s}{r \cdot \alpha \Delta \phi} \cdot \frac{1}{(1+\gamma)}
$$

$$
\left\{\because \quad s=n s^{\prime}\right\}
$$

Now if we calculate kinetic energy from here, we get-

$$
\begin{gathered}
E_{k}=\frac{1}{2} \psi \cdot\left(v_{p}^{\prime}\right)^{2}=\frac{1}{2} \psi \cdot \frac{s^{2} \cdot v_{p}^{2}}{\psi^{2} \cdot r^{2}(\alpha \Delta \phi)^{2}} \\
E_{k}=\frac{1}{2} \cdot \frac{s^{2}}{\gamma^{2} \cdot \psi^{2} \cdot r^{2}}
\end{gathered}
$$

Now by equation 19 , we get-

$$
v_{p}^{\prime}=v_{p}+v_{\phi}
$$

$$
\text { Here } \vec{v}_{\phi}=k_{\phi} \cdot \overrightarrow{\mathrm{F}}\left(\Phi_{B}\right)+k_{\phi}^{\prime} \cdot \overrightarrow{\mathrm{F}}\left(\Phi_{u}\right)
$$

We can say as flow determination velocity.

From equation 123, we can write-

$$
v_{p}=v_{p}^{\prime}-v_{\phi}
$$

$$
E_{\psi_{p}}=\psi_{p}^{2}
$$

$$
E_{\Delta \psi}=(\alpha \Delta \phi)^{2}=(\Delta \psi)^{2}
$$

$$
E_{\Delta \phi}=(\Delta \phi)^{2}=\left(\frac{\Delta \psi}{\alpha}\right)^{2}
$$

$$
\left(E_{p}\right)_{\psi}=\alpha . \psi_{p .} \phi=\psi_{p .} \psi^{\prime}=\alpha . E_{p}
$$

$$
E_{\xi}=\psi_{p .} \alpha \Delta \phi
$$

$$
\left(E_{c}\right)_{\phi}=\phi \cdot \Delta \phi=E_{c} / 2
$$

$$
\left(E_{c}\right)_{\psi}=\alpha^{2} \phi \Delta \phi=\alpha E_{c}=\alpha^{2}\left(E_{c}\right)_{\phi}
$$

$$
\left.\left(E_{p}\right)_{\psi}=\psi^{2}=\psi \psi^{\prime} \cdot \frac{\psi}{\psi^{\prime}}=E_{\psi} / \beta \quad \because \beta=\frac{\alpha \phi}{\psi}=\frac{\phi}{\phi^{\prime}}\right\}
$$

$$
\left(E_{p}\right)_{\phi}=\phi^{2}=\phi \phi^{\prime} \cdot \frac{\phi}{\phi^{\prime}}=\beta E \phi
$$

$$
\begin{aligned}
\left(E_{p}\right)_{c}=\psi \cdot \Delta \phi= & \left(\psi_{p}+\alpha \Delta \phi\right) \Delta \phi=\psi_{p} \Delta \phi+\alpha(\Delta \phi)^{2} \\
& =\frac{E_{\xi}}{\alpha}+\alpha E_{\Delta \phi}
\end{aligned}
$$

$$
E_{c}=\alpha \phi \cdot \Delta \phi=\psi^{\prime} \Delta \phi=\psi \Delta \phi \cdot \frac{\psi^{\prime}}{\psi}=\beta\left(E_{\beta}\right)_{c}
$$

These energies are interrelated with each other, like-

$$
\frac{E_{\Delta \psi}}{E_{\psi_{p}}}=\frac{(\alpha \Delta \phi)^{2}}{\left(\psi_{p}\right)^{2}}=\gamma^{2}
$$

So, we can write above equation, as

\subsection{Various Energy Terms}


Now by multiplying $E_{c}$ with, $\beta$ we get-

$$
\begin{aligned}
\beta E_{c}=\frac{\psi^{\prime}}{\psi} \cdot \alpha \phi \cdot \Delta \phi & =\frac{\alpha^{2} \phi^{2} \cdot \psi \Delta \phi}{\psi^{2}}=\frac{\alpha \phi^{2}(\psi \cdot \alpha \Delta \phi)}{\psi^{2}} \\
& =\frac{\alpha^{2}\left(E_{\beta}\right)_{\phi} \cdot\left(E_{\beta}\right)_{c}}{\left(E_{\beta}\right)_{\phi}}
\end{aligned}
$$

Now by using equation (133) and (134), we get (139) as-

$$
\beta E_{c}=\frac{\alpha^{2} \beta^{2} E_{\phi}\left(E_{\beta}\right)_{c}}{E_{\psi}}=\frac{\alpha^{2} \beta^{2} E_{\phi}\left(E_{\beta}\right)_{c}}{\alpha^{2} E_{\psi}}=\beta^{2}\left(E_{\beta}\right)_{c}
$$

Now there is a relation between $E_{\psi_{p}}$ and $E_{\xi}$ as-

$$
\frac{E_{\xi}}{E_{\psi_{p}}}=\frac{\psi_{p} \alpha \Delta \phi}{\psi_{p}^{2}}=\beta
$$

Or we can write above equation, as-

$$
E_{\xi}=\beta E_{\psi_{p}}
$$

So, these energies are interrelated with some relations as $\alpha, \beta, \gamma$ or we can define each energy form in another energy form by using these three parameters $\alpha, \beta, \gamma$. Now by Generalized Correspondence Principle power formerly defined energies in physics can be justified in form of these energy forms. Schrodinger defined his wave equation in contrast of De-Broglie Wave-Particle Duality. As I have described the Wave-Particle Duality from $\phi-\psi$ transformation or Wave-Particle Duality is a form of $\phi-\psi$ transformation. So, Schrodinger's wave equation can be defined in terms of my theoretical perspective.

\subsection{Forces in Universal Relativity}

At first I am clearing some facts like we know the force calculated by me in my former paper [5] as-

$$
\begin{gathered}
\vec{\delta}=\frac{\partial \vec{F}}{\partial \tau}+\frac{\partial \vec{F}}{\partial \phi} \\
\vec{F}=\psi \cdot \phi_{\text {covered }}=\psi \frac{\partial \vec{s}}{\partial \tau}
\end{gathered}
$$

Here $\vec{S}$ is vector form of line element in my theoretical perspective.

$$
v_{p}^{\prime}=v_{p}+K_{\phi} \cdot \mathrm{F}\left(\Phi_{B}\right)+k_{\phi}^{\prime} \cdot \mathrm{F}\left(\Phi_{u}\right)=\frac{\partial \vec{s}}{\partial \tau}
$$

As we know is the velocity measured by a static observer in universal scalar field $\left(\Phi_{u}\right)$ from the perspective of my predefined universal Principle of relativity. As we know by the definition of $\Phi_{M}^{0}$ that it governs the dynamics of Universe from my former paper [6]. So, we can write velocity as-

$$
\mathrm{F}\left(\Phi_{u}\right) \cong \mathrm{F}\left(\Phi_{M}^{0}\right)=\Gamma\left(\Phi_{M}^{0}\right)+\text { Other terms }
$$

Now by neglecting other terms, we get-

$$
\mathrm{F}\left(\Phi_{u}\right) \cong \Gamma\left(\Phi_{M}^{0}\right)
$$

$$
\Gamma\left(\Phi_{M}^{0}\right)=\frac{1}{\Phi_{M}^{0}} \cdot \frac{\partial \Phi_{M}^{0}}{\partial \tau}
$$

As we know-

$$
\frac{d s^{\prime}}{d \tau}=v_{p}^{\prime}=\frac{d s^{\prime} . \Gamma(\Phi) \Phi}{d \Phi}\left\{\because d \tau=\frac{d \Phi}{\Gamma(\Phi) \Phi}\right\}
$$

Now by using value of $F\left(\Phi_{u}\right)$ from equation [147] in equation [145], we get-

$$
\begin{gathered}
v_{p}^{\prime}=\frac{\partial \vec{s}}{\partial \tau}+k_{\phi} \cdot \mathrm{F}\left(\Phi_{B}\right)+k_{\phi}^{\prime} \cdot \mathrm{F}\left(\Phi_{u}\right) \\
\left\{\text { here } v_{p}=\frac{\partial s}{\partial \tau}\right\}
\end{gathered}
$$

We can also write the third term as velocity by Universe as-

$$
v_{u}=\frac{d s_{u}}{d \tau}=k_{\phi}^{\prime} \Gamma\left(\Phi_{M}^{0}\right)
$$

Now by putting the value of in above equation, we get-

$$
v_{u}=\frac{d s_{u}}{d \Phi_{M}^{0}} \cdot \Phi_{M}^{0} \cdot \Gamma\left(\Phi_{M}^{0}\right)=k_{\phi}^{\prime} \Gamma\left(\Phi_{M}^{0}\right)
$$

From above equation, we get value of $k_{\phi}^{\prime}$ as- 


$$
k_{\phi}^{\prime}=\Phi_{M}^{0} \cdot \frac{\partial s_{u}}{\partial \Phi_{M}^{0}}
$$

In similar way the flow of body also defined as-

$$
v_{B}=\frac{\partial s_{B}}{\partial \tau}=k_{\phi} \cdot \mathrm{F}\left(\Phi_{B}\right)=k_{\phi} \Gamma\left(\Phi_{B}\right)
$$

Or we get the value of $k_{\phi}$ as-

$$
k_{\phi}=\Phi_{B} \cdot \frac{\partial s_{B}}{\partial \Phi_{B}}
$$

So, dimension of is of space parameter and dimension of flow is inverse of time parameter. For different flows types of values and dimensions of varies.

Now the force part also can be modified as-

$$
\left[\frac{\partial F}{\partial \tau}\right]=\left[k_{\delta}\left[\frac{\partial F}{\partial \phi}\right]\right]
$$

Here $K_{\delta}$ is the dimensional equality parameter-

$$
\begin{gathered}
{[d \phi]=\left[k_{\delta}\right][d \tau]} \\
(157) \\
{[d \phi]=\left[k_{\delta}\right]\left[\frac{d \phi}{\Phi \Gamma(\Phi)}\right]}
\end{gathered}
$$

So, the equation [143] can be written as-

$$
\vec{\delta}=\frac{\partial \vec{F}}{\partial \tau}+k_{\delta} \cdot \frac{\partial \vec{F}}{\partial_{\phi}}
$$

So, my former equations needs to coefficient of force. The first term is purely Newtonian if $\left(F=\psi_{p} v_{p}\right)$ and Einstein's special relativity is added in $\left(F=\psi . v_{p}^{\prime}\right)$ but the second term is completely different and unforgettable in N-Time Inflationary Model of Universe. Now by putting the value of and in equation [150], we get-

$$
\frac{\partial s^{\prime}}{\partial \tau}=\frac{\partial s}{\partial \tau}+\Phi_{B} \cdot \frac{d s_{B}}{d \Phi_{B}} \cdot \mathrm{F}\left(\Phi_{B}\right)+\frac{d s_{u}}{d \Phi_{M}^{0}} \cdot \Phi_{M}^{0} \cdot \Gamma\left(\Phi_{M}^{0}\right)
$$

Now by multiplying above equation with $d \tau$, we get-

$$
d s^{\prime}=d s+\Phi_{B} \cdot \frac{d s_{B}}{d \Phi_{B}} \cdot \mathrm{F}\left(\Phi_{B}\right) \cdot d \tau+\frac{d s_{u}}{d \Phi_{M}^{0}} \cdot \Phi_{M}^{0} \cdot \Gamma\left(\Phi_{M}^{0}\right) \cdot d \tau
$$

$$
\because d \tau=\frac{d \Phi_{B}}{\Gamma\left(\Phi_{B}\right) \Phi_{B}} \text { and } d \tau=\frac{d \Phi_{M}^{0}}{\Gamma\left(\Phi_{M}^{0}\right) \cdot \Phi_{M}^{0}}
$$

So, the above equation becomes-

$$
\begin{aligned}
d s^{\prime}=d s+\Phi_{B} \cdot & \frac{d s_{B}}{d \Phi_{B}} \cdot \mathrm{F}\left(\Phi_{B}\right) \frac{d \Phi_{B}}{\Gamma\left(\Phi_{B}\right) \Phi_{B}} \\
& +\frac{d s_{u}}{d \Phi_{M}^{0}} \cdot \Phi_{M}^{0} \cdot \Gamma\left(\Phi_{M}^{0}\right) \cdot \frac{d \Phi_{M}^{0}}{\Gamma\left(\Phi_{M}^{0}\right) \cdot \Phi_{M}^{0}}
\end{aligned}
$$

$$
\because \mathrm{F}\left(\Phi_{B}\right) \cong \Gamma\left(\Phi_{B}\right)
$$

Now we get-

$$
d s^{\prime}=d s+d s_{B}+d s_{u}
$$

By integrating both terms-

$$
\int d s^{\prime}=\int d s+\int d s_{B}+\int d s_{u}+C
$$

\{Here $C$ is integration coefficient $\}$

$$
s^{\prime}=s+s_{B}+s_{u}+C
$$

Here $s_{B}$ and $s_{u}$ are respectively distance Disturbed by the flow of body and Universe. Now one query must be hitting your mental lexicon that if Universal Relativity exists then why we don't measure its effect on earth? The answer lies in the Principle itself that we are Observer at same body. Now take it in this way let's suppose an observer is observing two bodies with velocity $v_{1}^{\prime}$ and $v_{2}^{\prime}$ from the same body as-

$$
v_{1}^{\prime}=v_{1}+k_{\phi} \cdot \mathrm{F}\left(\Phi_{B}\right)+k_{\phi}^{\prime} \cdot \mathrm{F}\left(\Phi_{u}\right)
$$

And

$$
v_{2}^{\prime}=v_{2}+k_{\phi} \cdot \mathrm{F}\left(\Phi_{B}\right)+k_{\phi}^{\prime} \cdot \mathrm{F}\left(\Phi_{u}\right)
$$


Here the $2^{\text {nd }}$ and $3^{\text {rd }}$ both velocities are influenced by same body's flow and interaction with universal scalar field. So, their relative velocity will be-

$$
\begin{aligned}
v_{2-1}^{\prime}=v_{2}^{\prime}-v_{1}^{\prime}= & v_{2}-v_{1}+k_{\phi} \cdot \mathrm{F}\left(\Phi_{B}\right)-k_{\phi} \cdot \mathrm{F}\left(\Phi_{B}\right) \\
& +k_{\phi}^{\prime} \cdot \mathrm{F}\left(\Phi_{u}\right)-k_{\phi}^{\prime} \cdot \mathrm{F}\left(\Phi_{u}\right)=v_{2}-v_{1}
\end{aligned}
$$

So, here we only observe Galileo's relativity absolutely but if the velocities are near the critical speed $v_{c}$ then both bodies have second terms different and we will also measure the effect of second term (or Special Relativistic Case). So, on earth all bodies are influenced same as same as earth. So, we can't measure the effect of "Universal Relativity" but if both bodies are in different Central Systems over on different type of bodies not influenced by Universe in same way, then-

$$
v_{1}^{\prime}=v_{1}+k_{\phi_{1}} \cdot \mathrm{F}\left(\Phi_{B_{1}}\right)+k_{\phi_{1}}^{\prime} \cdot \mathrm{F}_{1}\left(\Phi_{u}\right)
$$

And for second body-

$$
v_{2}^{\prime}=v_{2}+k_{\phi_{2}} \cdot \mathrm{F}\left(\Phi_{B_{2}}\right)+k_{\phi_{2}}^{\prime} \cdot \mathrm{F}_{2}\left(\Phi_{u}\right)
$$

Now, in this case the relative velocity between them is-

$$
\begin{aligned}
v_{2-1}^{\prime}=v_{2}^{\prime}-v_{1}^{\prime}= & v_{2}-v_{1}+k_{\phi_{2}} \cdot \mathrm{F}\left(\Phi_{B_{2}}\right)-k_{\phi_{1}} \cdot \mathrm{F}\left(\Phi_{B_{1}}\right) \\
& +k_{\phi_{2}}^{\prime} \cdot \mathrm{F}_{2}\left(\Phi_{u}\right)-k_{\phi_{1}}^{\prime} \cdot \mathrm{F}_{1}\left(\Phi_{u}\right) \\
& =\left(v_{2}-v_{1}\right) \\
& +\left\{k_{\phi_{2}} \cdot \mathrm{F}\left(\Phi_{B_{2}}\right)-k_{\phi_{1}} \cdot \mathrm{F}\left(\Phi_{B_{1}}\right)\right\} \\
& +\left\{k_{\phi_{2}}^{\prime} \cdot \mathrm{F}_{2}\left(\Phi_{u}\right)-k_{\phi_{1}}^{\prime} \cdot \mathrm{F}_{1}\left(\Phi_{u}\right)\right\}
\end{aligned}
$$

Here in this case we will clearly see the effect of "Universal Relativity". This happened for example one body is far away from earth formed out from different coupling material and another one is on earth.

Now if the flow function is not equal to the evolution factor, then equation (160) will be-

$$
\frac{d s^{\prime}}{d \tau}=\frac{d s}{d \tau}+k_{\phi} \cdot \mathrm{F}\left(\Phi_{B}\right)+k_{\phi}^{\prime} \cdot \mathrm{F}\left(\Phi_{u}\right)
$$

Now by multiplying both sides with $d \tau$ and integrating them, we get-

$$
s^{\prime}=s+\int k_{\phi} \cdot \mathrm{F}\left(\Phi_{B}\right) \cdot d \tau+\int k_{\phi}^{\prime} \cdot \mathrm{F}\left(\Phi_{u}\right) \cdot d \tau+C
$$

Now by comparing above equation with equation (164), we get-

$$
\begin{array}{r}
s_{B}=\int k_{\phi} \cdot F\left(\Phi_{B}\right) \cdot d \tau \\
s_{u}=\int k_{\phi}^{\prime} \cdot F\left(\Phi_{u}\right) \cdot d \tau
\end{array}
$$

So, the difference between $s^{\prime}$ (apparent) and $s$ (actual) can be defined as-

$$
\begin{aligned}
s^{\prime}-s=s_{\text {affected }} & =s^{\prime \prime}=s_{B}+s_{u} \\
& =\int k_{\phi} \cdot \mathrm{F}\left(\Phi_{B}\right) \cdot d \tau+\int k_{\phi}^{\prime} \cdot \mathrm{F}\left(\Phi_{u}\right) \cdot d \tau+C
\end{aligned}
$$

Similarly $v_{\text {affected }}$ can be defined as-

$$
v_{\text {affected }}=v^{\prime}-v=v^{\prime \prime}=k_{\phi} \cdot \mathrm{F}\left(\Phi_{B}\right)+k_{\phi}^{\prime} \cdot \mathrm{F}\left(\Phi_{u}\right)
$$

Now in similar way broken parts also affected by Universal Relativity. Suppose a broken part is travelling from different flows hope universal scalar field without any interruption of body, then we can represent it as-

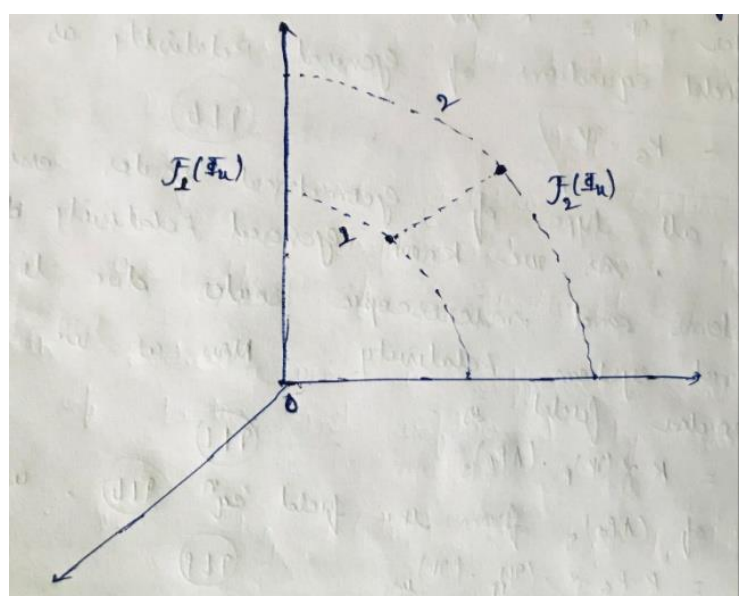

FIG. 4 Geometrical Representation of Broken Parts in Universal Relativity

In this case if-

$$
\mathrm{F}_{1}\left(\Phi_{u}\right) \neq \mathrm{F}_{2}\left(\Phi_{u}\right)
$$

Then-

$$
\left(v_{1}^{\prime}\right)_{b}=\left(v_{1}\right)_{b}+k_{\phi_{1}}^{\prime} \mathrm{F}_{1}\left(\Phi_{u}\right)
$$




$$
\left(v_{2}^{\prime}\right)_{b}=\left(v_{2}\right)_{b}+k_{\phi_{2}}^{\prime} \mathrm{F}_{2}\left(\Phi_{u}\right)
$$

Now according to special relativity $\left(v_{1}^{\prime}\right)_{b}-\left(v_{2}^{\prime}\right)_{b}=c-c=$ 0 because, the speed of light or broken parts is universal constant. But in this case-

$$
\begin{aligned}
\left(v_{1}^{\prime}\right)_{b}-\left(v_{2}^{\prime}\right)_{b}= & \left\{\left(v_{1}\right)_{b}-\left(v_{2}\right)_{b}\right\} \\
& +\left\{k_{\phi_{1}}^{\prime} \mathrm{F}_{1}\left(\Phi_{u}\right)-k_{\phi_{2}}^{\prime} \mathrm{F}_{2}\left(\Phi_{u}\right)\right\} \neq 0
\end{aligned}
$$

If $\mathrm{F}_{1}\left(\Phi_{u}\right) \neq \mathrm{F}_{2}\left(\Phi_{u}\right)$ or condition (178) is implied. So, foundation of special relativity is quite wrong in sense of Universal Relativity. In case of Doppler Effect [25] same things happens because-

$$
\left(v_{b}\right)_{c}=v \lambda
$$

If $\left(v_{1}^{\prime}\right)_{b}>\left(v_{2}^{\prime}\right)_{b}$ then $\lambda_{1}>\lambda_{2}$ or we measure red shift in a straight line.

\subsection{Generalization of Theory of General Relativity in Universal Mechanics}

Now I am defining same quantities which are key points to describe General Relativity in terms of my Theoretical perspective. These quantities are usually gradient, divergence and curl of scalar field functions $(\Phi)$ like scalar field density $\left(\rho_{\phi}\right)$ and quantity in a body $(\psi)$. Usually curvature in spacetime is straightforwardly related to variation in scalar field density. So, the field equations of General Relativity [12] can be written as-

$$
R_{\mu \nu}-\frac{1}{2} g_{\mu \nu} R=G_{\mu \nu}=k T_{\mu \nu}
$$

Curvature in Space Time $\equiv$ Matter

As we know from my above description of curvature, we can write it as-

$$
\begin{aligned}
& \Delta \rho_{\phi} \equiv \text { variation in scalar field density } \\
& \qquad \text { Curvature in Space Time }=G_{\mu \nu}
\end{aligned}
$$

Or matter can be written as-

$$
\text { Matter }=\psi \equiv k T_{\mu \nu}
$$

So, we can write field equations of General Relativity as-

$$
\Delta \rho_{\phi}=K_{G} \cdot \psi
$$

This equation is valid for all type of geometrical skills exists in Universe. (Like for items also). As we know General Relativity does not describe the atomic Central Systems and microscopic skills due to not including the Principle of Central System Relativity. Now as we know the force by variation in scalar field is-

$$
\delta_{\rho_{\phi}}=k \alpha(\psi)_{p e r} \cdot\left(\Delta \rho_{\phi}\right)_{s}
$$

Now by putting the value of $\left(\Delta \rho_{\phi}\right)_{S}$ from the field equation [187], we get equation [188] as-

$$
\delta_{\rho_{\phi}}=k \cdot K_{G} \alpha(\psi)_{\text {per }}(\psi)_{\text {in }}
$$

For $(\psi)_{\text {per }} \rightarrow m_{1}$ and $(\psi)_{\text {in }} \rightarrow m_{2}$ this force intent towards the Newton's law of gravitation and also describes General Relativity by some normal limits. Now the gradient shows the maximum value of a scalar field along each direction as-

$$
\nabla \Phi\left(u_{1}, u_{2}, \ldots ., u_{n}\right)
$$

Here $u_{1}, u_{2}, \ldots ., u_{n}$ are the orthonormal basis vectors on and n- dimensional manifold.

$$
\nabla \rho_{\phi}=\nabla\left(\frac{\Phi}{\mathcal{V}\left(\mathcal{M}^{\mathrm{n}}\right)}\right)
$$

For above and n-dimensional manifold this can be written in terms of n-orthonormal basis vectors as-

$$
\nabla \rho_{\phi}=\nabla\left(\frac{\Phi\left(u_{1}, u_{2}, \ldots, u_{n}\right)}{\mathcal{V}\left(\mathcal{M}^{\mathrm{n}}\right)}\right)
$$

$$
\left\{\because \nabla\left(\frac{f}{g}\right)=-\frac{(f \nabla g-g \nabla f)}{g^{2}}\right\}
$$

$\nabla \rho_{\phi}$

$=\frac{\mathcal{V}\left(\mathcal{M}^{\mathrm{n}}\right) \nabla \Phi\left(u_{1}, u_{2}, \ldots \ldots, u_{n}\right)-\Phi\left(u_{1}, u_{2}, \ldots, u_{n}\right) \nabla \mathcal{V}\left(\boldsymbol{M}^{\mathrm{n}}\right)}{\nabla \mathcal{V}\left(\boldsymbol{M}^{\mathrm{n}}\right)^{2}}$

In similar way curl of $\nabla \Phi\left(u_{1}, u_{2}, \ldots \ldots, u_{n}\right)$ can be used to describe spin and spin generated force or flow of scalar field as- 


$$
\nabla \times \nabla \Phi\left(u_{1}, u_{2}, \ldots ., u_{n}\right)
$$

Similarly we can define-

$$
\nabla \times \nabla \rho_{\phi}
$$

For Newton's approximation-

$$
F_{G}=\frac{G \cdot m_{1} m_{2}}{r^{2}}=k K_{G} \alpha \cdot m_{1} m_{2}=\nabla \rho_{\phi}
$$

Or we can justify-

$$
k K_{G} \alpha=\frac{G}{r^{2}}
$$

Now equation [187] can be written as-

$$
\nabla\left(\frac{\Phi}{\mathcal{V}\left(\mathcal{M}^{\mathrm{n}}\right)}\right)=K_{G} \psi
$$

As we know $\mathcal{V}\left(\boldsymbol{M}^{\mathrm{n}}\right)=\frac{4}{3} \pi r^{3}$ is for spherical-symmetry in Newtonian limit.

$$
\left(\frac{\nabla \Phi}{\frac{4}{3} \pi r^{3}}\right)-\frac{\left(4 \pi r^{2} \cdot \Delta r\right) \Phi}{\left(\frac{4}{3} \pi r^{3}\right)^{2}}=K_{G} \psi
$$

$$
\frac{\Delta \Phi}{\mathcal{V}}-\frac{3\left(\frac{\Delta r}{r}\right) \Phi}{\mathcal{V}\left(\mathcal{M}^{\mathrm{n}}\right)}=K_{G} \psi
$$

Now by putting $\psi=\psi_{p}+\alpha \Delta \Phi-$

$$
\Delta \Phi-3\left(\frac{\Delta r}{r}\right) \Phi=K_{G}\left(\frac{4}{3} \pi r^{3}\right)\left(\psi_{p}+\alpha \Delta \Phi\right)
$$

Now by some manipulations-

$$
\Delta \Phi\left(1-K_{G} \mathcal{V} \alpha\right)=K_{G} \mathcal{V} \psi_{p}+3\left(\frac{\Delta r}{r}\right) \Phi
$$

$$
K_{G}=\frac{\Delta \Phi-3\left(\frac{\Delta r}{r}\right) \Phi}{\mathcal{V} \psi}
$$

So, have dimensions of $K_{G^{-}}$

$$
\frac{1}{\operatorname{dim}[\mathcal{V}] \cdot \operatorname{dim}[\alpha]}
$$

Now by putting the value in Newtonian limit in equation (197), we get-

$$
k . \frac{\Delta \Phi-3\left(\frac{\Delta r}{r}\right) \Phi}{V \psi} \alpha=\frac{G}{r^{2}}
$$

As we know Newton did not considered $\phi-\psi$ transformation. So, we can write above equation as-

$$
-k .3\left(\frac{\Delta r}{r}\right) \frac{\alpha \Phi}{\psi}=\frac{G \times \frac{4}{3} \pi r^{3}}{r^{2}}
$$

$$
\left\{\because \frac{\alpha \Phi}{\psi}=\beta\right\}
$$

So, above equation becomes-

$$
-K .\left(\frac{\Delta r}{r}\right) \beta=G \frac{4}{9} \pi r
$$

Or

$$
\frac{K \beta}{G}=\left(\frac{2}{3} r\right)^{2} \frac{\pi}{(-\Delta r)} \quad\left\{\because A=4 \pi r^{2}\right\}
$$

$$
\frac{k \beta}{G}=-\frac{A}{9 \Delta r}
$$

Now I am defining an another beautiful aspect of Einstein field equations in my theoretical perspective, as -

$$
\psi \equiv k T_{\mu \nu}=\frac{8 \pi G}{c^{4}} T_{\mu \nu}=K_{T} \psi
$$

$$
G_{\mu \nu} \equiv \Delta \rho_{\phi}=G_{\mu \nu}=K_{R} \Delta \rho_{\phi}
$$

From here- 


$$
G_{\mu \nu}=\frac{8 \pi G}{c^{4}} T_{\mu \nu}=K_{R} \Delta \rho_{\phi}=K_{T} \psi
$$

So,

$$
\Delta \rho_{\phi}=\left(\frac{K_{R}}{K_{T}}\right) \psi
$$

Now by comparing equation [187] with [212], we get-

$$
K_{G}=\frac{K_{R}}{K_{T}}
$$

Now by calculating the force by variation in density of scalar field, we get-

$$
\delta_{\rho_{\phi}}=k \cdot \alpha(\psi)_{m}\left(\Delta \rho_{\phi}\right)_{c}
$$

Here is the quantity of moving body in scalar field of central body or moving by variation in the scalar field density of central body $\left(\Delta \rho_{\phi}\right)_{c}$. Now for $\mathrm{n}$ - dimensional manifold structure--

$$
\delta_{\rho_{\phi}}=k \cdot K_{G} \alpha(\psi)_{m}(\psi)_{c}
$$

Now putting the value of $K_{G}$ as-

$$
\begin{aligned}
K_{G}=\frac{\Delta \rho_{\phi}}{\psi} & =\frac{\mathcal{V}\left(\boldsymbol{M}^{\mathrm{n}}\right) \Delta \Phi\left(u_{1}, u_{2}, \ldots ., u_{n}\right)-\Phi \Delta \mathcal{V}\left(\boldsymbol{M}^{\mathrm{n}}\right)}{\psi \cdot \mathcal{V}^{2}\left(\mathcal{M}^{\mathrm{n}}\right)} \\
\delta_{\rho_{\phi}} & =k \cdot(\psi)_{m}\left\{\frac{\alpha \Delta \Phi}{\mathcal{V}\left(\mathcal{M}^{\mathrm{n}}\right)}-\alpha \Phi \cdot \frac{\Delta \mathcal{V}\left(\boldsymbol{M}^{\mathrm{n}}\right)}{\mathcal{V}^{2}\left(\mathcal{M}^{\mathrm{n}}\right)}\right\}
\end{aligned}
$$

Now-

$$
\delta_{\rho_{\phi}}=k \cdot\left(\psi_{p}+\alpha \Delta \Phi\right)_{m} \alpha K_{G} \cdot\left(\psi_{p}+\alpha \Delta \Phi\right)_{c}
$$

By solving further, we get-

$$
\begin{aligned}
\delta_{\rho_{\phi}}=k \cdot \alpha K_{G} \cdot\{( & \left.\psi_{p}\right)_{m}\left(\psi_{p}\right)_{c}+(\alpha \Delta \Phi)_{m}\left(\psi_{p}\right)_{c} \\
& \left.+\left(\psi_{p}\right)_{m}(\alpha \Delta \Phi)_{c}+(\alpha \Delta \Phi)_{m}(\alpha \Delta \Phi)_{c}\right\}
\end{aligned}
$$

Now by putting it in equation (217)-

$$
\begin{aligned}
\delta_{\rho_{\phi}}=k \cdot\left(\psi_{p}+\alpha\right. & \Delta \Phi)_{m}\left\{\frac{(\alpha \Delta \Phi)_{c}}{\mathcal{V}\left(\mathcal{M}^{\mathrm{n}}\right)}-(\alpha \Phi)_{c} \cdot \frac{\Delta \mathcal{V}\left(\mathcal{M}^{\mathrm{n}}\right)}{\mathcal{V}^{2}\left(\mathcal{M}^{\mathrm{n}}\right)}\right\} \\
& =\frac{k}{\mathcal{V}\left(\boldsymbol{M}^{\mathrm{n}}\right)}\left\{\left(\psi_{p}\right)_{m}(\alpha \Delta \Phi)_{c}\right. \\
& +(\alpha \Delta \Phi)_{m}(\alpha \Delta \Phi)_{c} \\
& +\beta_{c}\left(\left(\psi_{p}\right)_{m}(\psi)_{c}\right. \\
& \left.\left.+(\alpha \Delta \Phi)_{m}(\psi)_{c}\right) \frac{\Delta \mathcal{V}\left(\mathcal{M}^{\mathrm{n}}\right)}{\mathcal{V}\left(\boldsymbol{M}^{\mathrm{n}}\right)}\right\}
\end{aligned}
$$

$$
\left\{\because \beta_{c}=\frac{(\alpha \Phi)_{c}}{(\psi)_{c}}\right\}
$$

Now by solving further to above equation, we get-

$$
\begin{aligned}
\delta_{\rho_{\phi}}=\frac{k}{\mathcal{V}\left(\mathcal{M}^{\mathrm{n}}\right)}\{ & \left(\psi_{p}\right)_{m}(\alpha \Delta \Phi)_{c}+(\alpha \Delta \Phi)_{m}(\alpha \Delta \Phi)_{c} \\
& +\beta_{c} \frac{\Delta \mathcal{V}\left(\mathcal{M}^{\mathrm{n}}\right)}{\mathcal{V}\left(\boldsymbol{M}^{\mathrm{n}}\right)}\left(\left(\psi_{p}\right)_{m}\left(\psi_{p}\right)_{c}\right. \\
& +(\alpha \Delta \Phi)_{m}\left(\psi_{p}\right)_{c}+\left(\psi_{p}\right)_{m}(\alpha \Delta \Phi)_{c} \\
& \left.\left.+(\alpha \Delta \Phi)_{m}(\alpha \Delta \Phi)_{c}\right)\right\}
\end{aligned}
$$

Now by comparing equation (219) with equation (221), we get-

$$
\begin{aligned}
\alpha K_{G} \mathcal{V}\left(\mathcal{M}^{n}\right)= & \left\{\frac{\left(\psi_{p}\right)_{m}(\alpha \Delta \Phi)_{c}+(\alpha \Delta \Phi)_{m}(\alpha \Delta \Phi)_{c}}{(\psi)_{m}(\psi)_{c}}\right\} \\
& -\beta_{c}\left(\frac{\Delta \mathcal{V}\left(\mathcal{M}^{\mathrm{n}}\right)}{\mathcal{V}\left(\boldsymbol{M}^{\mathrm{n}}\right)}\right)
\end{aligned}
$$

For every kind of Central System or geometries this equation holds and we can define value of $K_{G}$ for different kinds of Central Systems (like for atomic, solar, galactic etc.). So, In case of Atomic Central Systems also, gravitational like force plays a vital role but for different from solar systems case. So, Bohr's atomic model is incomplete for does not includes the density variation force instead of electromagnetic force. Now I have defined charged age quantity of imbalance only and as manifestation of geometry in Central Systems or bodies. So, electromagnetic force does not plays a vital role in formation and stability of Atomic Central Systems it is the only $\delta_{\rho_{\phi}}$ or scalar field density variation force on different scales which is responsible for formation and stability of Central Systems in Universe.

Now according to Newton's law of gravitation if two bodies are in space, than the force will be- 


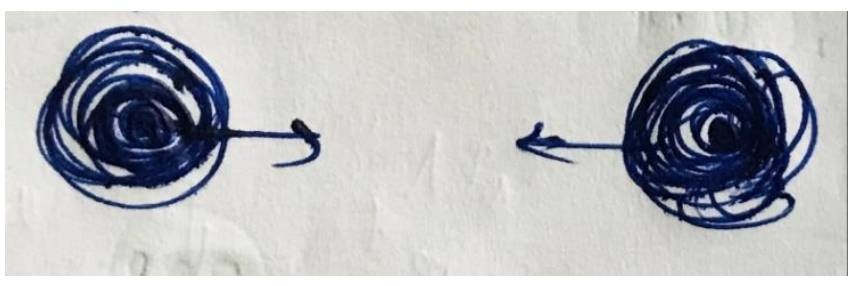

FIG. 5 Geometrical Representation of Two Bodies Attracted by each other

In this situation both bodies will collide but this never happens in real cases because this force is a manifestation of scalar field density variation around bodies.

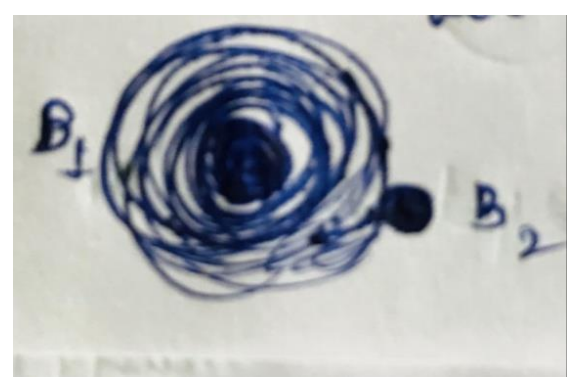

FIG. 6 Geometrical Representation of Bodies under Central System Force

Here in this case body $\mathrm{B}_{2}$ will move around body $\mathrm{B}_{1}$ because the minimal coupling of body $\mathrm{B}_{1}$ 's scalar field and it forms a particular geometry round central body. According to Newton if both bodies will collide, then how formation of Central Systems is possible in Universe? As I described in my $3^{\text {rd }}$ paper [3] a perfect body always choose the path which is in same scalar field coupling state as the body have. So, in my theoretical perspective this will happen to both bodies $\mathrm{B}_{1}$ and $\mathrm{B}_{2}$ as-

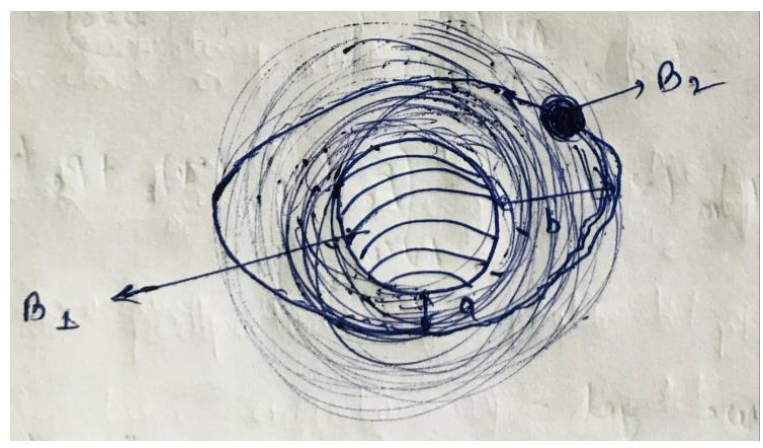

FIG. 7 Geometrical Representation of Stability of Bodies in Central System Force

Here is the minimum length of orbit which defines the body cannot reach the next level of a scalar field density variation around body. I defined in one of my former papers that this is impossible to collide two bodies of same type of scalar field with each other. So, the Central Systems are stable in this case.
Now by putting value of for Newtonian limit we can define $\delta_{\rho_{\phi}}$ equivalent to newtons law of gravitation as-

$$
\delta_{\rho_{\phi}}=-\frac{A G \alpha}{9 P_{c} \Delta r}(\psi)_{p}\left(\Delta \rho_{\phi}\right)_{c} \equiv-\frac{G m_{1} m_{2}}{r^{2}}
$$

Here negative value of force defines that nature of force is attractive. We can also define the stress energy momentum tensor part as-

$$
T_{\mu v}=K_{T}^{E}(\phi \psi)
$$

$$
R_{\mu v}-\frac{1}{2} g_{\mu v} R=K_{R} \Delta \rho_{\phi}=K T_{\mu v} \quad \because K=\frac{8 \pi G}{c^{4}}
$$

Now by putting the value of in equation [224] from equation [225], we get

$$
K_{R} \Delta \rho_{\phi}=\frac{8 \pi G}{c^{4}} \cdot K_{T}^{E}(\phi \psi)
$$

So, we get-

$$
\Delta \rho_{\phi}=\frac{8 \pi G}{c^{4}} \cdot \frac{K_{T}^{E}}{K_{R}}(\phi \psi)
$$

Here by putting speed of light as critical velocity, we get

$$
\Delta \rho_{\phi}=\frac{8 \pi G}{c^{4}} \cdot K_{G}^{\prime}(\phi \psi)
$$

Or

$$
\Delta \rho_{\phi}=K_{G}^{\prime \prime}(\phi \psi)
$$

Or

$$
\Delta \rho_{\phi}=G_{x} E
$$

Here $G_{x}$ is different for different Central Systems or $x=$ $s, a, g \ldots$ etc. Here stands for solar systems, stands for atomic Central Systems, stands for galactic Central Systems etc. By these equations we can also define energy as- 


$$
E=\frac{\Delta \rho_{\phi}}{G_{x}}
$$

Or

$$
\delta_{\rho_{\phi}}=K_{x} G_{x} \alpha(\psi)_{m}(E)_{c}
$$

So, this force is implied on both macroscopic and microscopic point of scales or valid in Quantum Mechanics and General Relativity both theoretical perspectives.

Defining Niels Bohr's reduced mass [26] as-

$$
\mu_{c}=\frac{m_{c}}{1+\frac{m_{c}}{m_{n}}}
$$

Now by putting in my terms to above equation, we get

$$
\left(\psi_{c}\right)_{r}=\frac{\psi_{e}}{1+\frac{\psi_{e}}{\psi_{c}}}
$$

Here $\left(\psi_{c}\right)_{r}$ reduced quantity of electron and quantity of electron $\psi_{c}$ or quantity of nucleus

Now by Generalized Correspondence Principle, the universal counterpart of the above equation-

$$
\left(\psi_{p e r}\right)_{r}=\frac{\psi_{p e r}}{1+\frac{(\psi)_{p e r}}{\psi_{c}}}
$$

Here is the reduced quantity of a perfect body or is the perfect quantity hope that perfect body and is the quantity of central body or perfect body. Now by solving for the two above equations (235), we get-

$$
\left(\psi_{p e r}\right)_{r}=\frac{\left(\psi_{p e r}\right) \psi_{c}}{\left(\psi_{p e r}\right)+\psi_{c}}
$$

Or

$$
\left(\psi_{\text {per }}\right)_{r}\left(\psi_{\text {per }}+\psi_{c}\right)=\left(\psi_{\text {per }}\right) \psi_{c}
$$

Now according to N-Time Inflationary Model mass or quantity of each electron is different for each other. But we find that mass of each electron is same yet period now if two electrons are in an atom as -

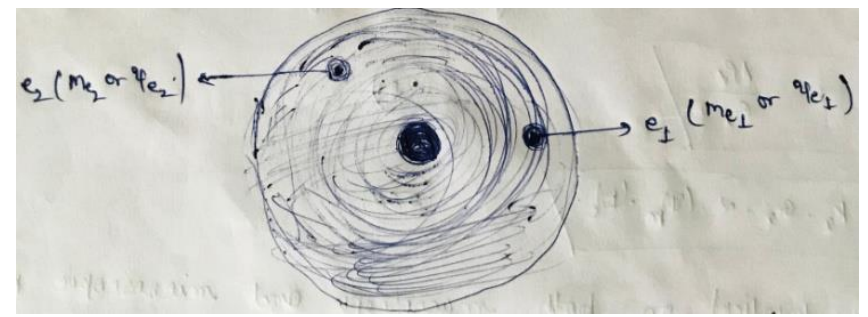

FIG. 8 Geometrical Representation of Atomic Central System

So, according to our former physical phenomenology-

$$
m_{c_{1}}=m_{c_{2}}=m_{c}
$$

Or

$$
\psi_{c_{1}}=\psi_{c_{2}}=\psi_{c}
$$

But according to my physical phenomenology, the mass or quantities of two electrons are not same, so-

$$
m_{c_{1}} \neq m_{c_{2}} \text { Or } \quad \psi_{c_{1}} \neq \psi_{c_{2}}
$$

Or there is always a difference between the masses or quantities of two different electrons. In other words we can define an outstanding fact from here that each and everybody or geometry exist in Universe have different quantity mass or inertia whether it is off same kind (like electrons, protons, planets, stars, Sub electrons, quarks, baryons Moons, galaxies, neutrons etc). There is an outcome comes from here that at now we were defining a particular quantity of bodies like for electrons $\left(9.1 \times 10^{-31} \mathrm{~kg}\right)$, For protons $\left(6.67 \times 10^{-27} \mathrm{~kg}\right)$ or for neutrons $\left(6.67 \times 10^{-27} \mathrm{~kg}\right)$ is a nice approximation but not particularly correct or-

$$
\psi_{c_{1}} \neq \psi_{c_{2}} \neq 9.1 \times 10^{-31} \mathrm{~kg}
$$

Now from equation [237], we get quantity of central body as-

$$
\left(\psi_{p e r}\right)_{r} \psi_{p e r}=\psi_{c}\left(\psi_{p e r}-\left(\psi_{p e r}\right)_{r}\right)
$$




$$
\psi_{c}=\frac{\left(\psi_{p e r}\right)_{r}}{1+\frac{\left(\psi_{p e r}\right)_{r}}{\psi_{p e r}}}
$$

Here one more fact can be added two above theorem that according N-Time to earn time inflationary modern of Universe the bodies formed in same universal inflations or epochs have quantities of same order.

$$
\psi_{e_{1}} \cong \psi_{e} \cong \psi_{e_{2}}
$$

So, at now in Quantum Mechanics we were only obtaining approximation in quantities of atomic or sub atomic particles not exactness. There is a relation between $K_{x}$ and $G_{x}$ as-

$$
\begin{gathered}
\Delta \rho_{\phi}=G_{x} E \\
\Delta \rho_{\phi}=K_{x} \psi \\
\frac{G_{x} E}{K_{x} \psi}=1 \\
G_{x} \phi=K_{G}
\end{gathered}
$$

Now from equation [228], we get-

$$
G_{x}=\frac{8 \pi G}{v_{c}{ }^{4}} \cdot K_{G}^{\prime}
$$

Here I defined the in my former consideration as a property of coupling of quantity with a scalar field $(\phi)$. So, that travelling speeds of gravitational waves also different in different densities of scalar fields. Now by putting value from equation [246] in equation [245], we get-

$$
\frac{8 \pi G \phi}{v_{C}{ }^{4}} \cdot \frac{K_{G}}{K_{G}^{\prime}}
$$

Now I intend to define in form of density of propagating scalar field as-

$$
v_{c} \propto\left(\rho_{\phi}\right)_{\text {travelling }}
$$

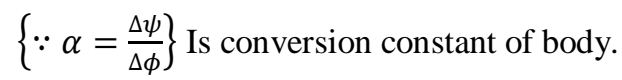

As we know at critical velocity $\phi-\psi$ transformation is maximum, so, $\propto$ will be maximum, by removing the proportionality with constant and combining both equations [248] end [249], we get-

$$
v_{c}=K_{c} \alpha_{\max }\left(\rho_{\phi}\right)_{\text {travelling }}
$$

Now as we know where is around a body. So, critical velocity also increase a wave going towards the body or travelling speed of gravitational wave will decrease from body too far space. We can clearly see this as-

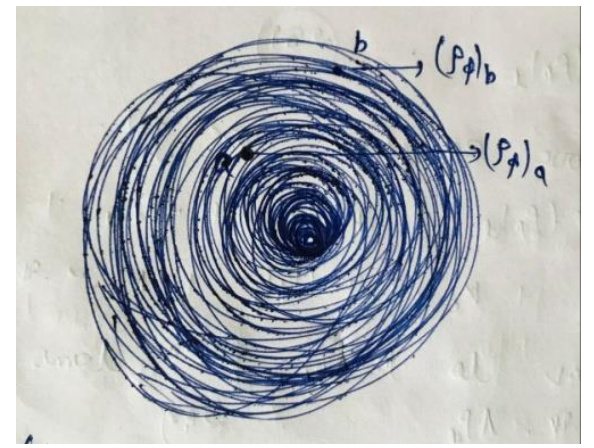

FIG. 9 Geometrical Representation of Variation in Speed of Gravitational Wave

$$
\begin{gathered}
\left(\rho_{\phi}\right)_{a}>\left(\rho_{\phi}\right)_{b} \quad \text { So, }\left(\rho_{\phi}\right)_{b \rightarrow a}=+v e \\
\text { So, } \quad\left(\Delta v_{c}\right)_{b \rightarrow a}=+v e
\end{gathered}
$$

Or $\Delta\left(\rho_{\phi}\right)_{a \rightarrow b}=-v e=\left(\rho_{\phi}\right)_{b}-\left(\rho_{\phi}\right)_{a}$ so, $\left(\Delta v_{c}\right)_{a \rightarrow b}=-v e$ or the speed of propagation hope gravitational wave will decrease from point A to point $b$. Now by putting value of $\alpha_{\max }$, we get equation [250] as-

$$
v_{c}=K_{c}\left(\frac{\Delta \psi}{\Delta \Phi}\right)\left(\rho_{\phi}\right)_{t}
$$

Now by multiplying both sides with quantity of that body which is travelling at critical speed in scalar field, we get-

$$
\psi v_{c}=K_{c} \psi\left(\frac{\Delta \psi}{\Delta \Phi}\right)\left(\rho_{\phi}\right)_{t}
$$

This can be written as-

$$
v_{c} \propto \alpha
$$




$$
F_{c}=K_{c} \psi\left(\frac{\Delta \psi}{\Delta \Phi}\right)\left(\rho_{\phi}\right)_{t}
$$

Now from equation (159), we get-

$$
\delta=K_{c} \frac{\partial F}{\partial \tau}+K_{g} \cdot \frac{\partial F}{\partial \phi}
$$

Here is the dimensional parameter with dimension $[\phi] /[\tau]$.

Or

$$
\delta=K_{c} \frac{\partial F}{\partial \tau} d \tau+\frac{\partial F}{\partial \phi} d \phi
$$

As I defined earlier, quantity of a body is totally convertible to scalar field at critical velocity. Cherenkov radiation effect indicates that electrons don't reach at critical velocity at speed of light or this proves that critical velocity can be different for different kind of bodies. At critical velocity, we can write-

$$
\psi=\alpha \Delta \Phi \quad \text { Or } \psi_{p}=0
$$

For $\phi-\psi$ transformation-

$$
\Delta \psi=\alpha \Delta \Phi
$$

Now by comparing both equations, at critical velocity we get-

$$
\psi=\Delta \psi
$$

Or by taking variation both sides, we get-

$$
\begin{gathered}
\Delta \psi=\Delta^{2} \psi \Rightarrow \Delta^{2} \psi=\psi \\
\Delta^{n} \psi=\psi
\end{gathered}
$$

Now equation [253] can be written as by using value from equation [256] as-

$$
F_{c}=K_{c} \frac{(\alpha \Delta \Phi)(\alpha \Delta \Phi)}{(\Delta \Phi)} \cdot\left(\rho_{\phi}\right)_{t}
$$

$$
F_{c}=K_{c} \alpha \psi \cdot\left(\rho_{\phi}\right)_{t}
$$

Now by taking variation in above equation, we get-

$$
\begin{gathered}
\Delta F_{c}=K_{c} \Delta \alpha \psi \cdot\left(\rho_{\phi}\right)_{t}+K_{c} \alpha \Delta \psi \cdot\left(\rho_{\phi}\right)_{t}+K_{c} \alpha \psi \cdot \Delta\left(\rho_{\phi}\right)_{t} \\
=K_{c} \Delta \alpha \psi \cdot\left(\rho_{\phi}\right)_{t}+K_{c} \alpha \psi \cdot\left(\rho_{\phi}\right)_{t} \\
+K_{c} \alpha \psi \cdot \Delta\left(\rho_{\phi}\right)_{t}
\end{gathered}
$$

Here's the third term is similar to force terms-

$$
\delta \rho_{\phi}=K_{c} \alpha \psi \cdot \Delta \rho_{\phi}
$$

Now I putting value in equation [254], we get-

$$
\delta=\frac{\partial F_{c}}{\partial \tau}+K_{g} \cdot \frac{\partial F_{c}}{\partial \phi}
$$

Now by solving further-

$$
\begin{aligned}
\delta=K_{c} \frac{\partial \alpha}{\partial \tau} \psi \cdot\left(\rho_{\phi}\right)_{t} & +K_{c} \alpha \frac{\partial \psi}{\partial \tau} \cdot\left(\rho_{\phi}\right)_{t}+K_{c} \alpha \psi \cdot \frac{\partial\left(\rho_{\phi}\right)_{t}}{\partial \tau} \\
& +K_{c} K_{\delta} \frac{\partial \alpha}{\partial \phi} \psi \cdot\left(\rho_{\phi}\right)_{t}+K_{c} K_{\delta} \alpha \frac{\partial \psi}{\partial \tau} \cdot\left(\rho_{\phi}\right)_{t} \\
& +K_{c} K_{\delta} \alpha \psi \cdot \frac{\partial\left(\rho_{\phi}\right)_{t}}{\partial \phi}
\end{aligned}
$$

Now as we know broken parts (light particles, photon etc) also travel at their critical speed. So, we can write $F_{c}=F_{b}$ for broken parts as-

$$
F_{c}=F_{b}=\psi_{b} v_{c}
$$

For same properties $\psi_{b}=\alpha \Delta \phi_{b}$ or $\Delta \psi_{b}=\alpha \Delta \phi_{b}$ as body at critical velocity follows. So, variation in the motion of a broken part can be written by equation [262] as-

$$
\Delta F_{b}=K_{c} \Delta \alpha \psi_{b} \cdot\left(\rho_{\phi}\right)_{t}+K_{c} \alpha \Delta \psi_{b} \cdot\left(\rho_{\phi}\right)_{t}+K_{c} \alpha \psi_{b} \cdot \Delta\left(\rho_{\phi}\right)_{t}
$$

Here first part is connected to geometry of light and second part is connected to loss in quantity but $3^{\text {rd }}$ part straightforwardly connected to change in motion of light or broken part by gravitational field (or created due to variation in the density of scalar field).

$$
\delta_{\rho_{\phi}}=K_{x} \alpha \psi_{b} \cdot\left(\Delta \rho_{\phi}\right)_{t}
$$


(268)

So, this is the beautiful reason behind the gravitational bending of light [28]. So, gravitational waves travel at critical velocity of a particular scalar field. Speed of gravitational waves [29] also varies according to variation in scalar field density-

$$
\Delta F_{b}=K_{c} \Delta \alpha \psi_{b} \cdot\left(\rho_{\phi}\right)_{t}+K_{c} \alpha \Delta \psi_{b} \cdot\left(\rho_{\phi}\right)_{t}+K_{c} \alpha \psi_{b} \cdot\left(\Delta \rho_{\phi}\right)_{t}
$$

So, we can write the force as-

$$
\delta_{\rho_{\phi}}=K_{c} \alpha \psi_{b} \cdot\left(\Delta \rho_{\phi}\right)_{t}
$$

Now by putting the value of $K_{c}$ from equation (250), we get-

$$
\delta_{\rho_{\phi}}=\frac{v_{c}}{\alpha_{\max }\left(\rho_{\phi}\right)_{t}} \alpha \psi_{b} \cdot\left(\Delta \rho_{\phi}\right)_{t}
$$

Now by using field equations-

$$
\begin{gathered}
\Delta \rho_{\phi}=G_{x} E \\
\Delta \rho_{\phi}=K_{c} \psi \\
\Delta \rho_{\phi}=\frac{8 \pi G}{v_{c}} K_{G}^{\prime} E
\end{gathered}
$$

We can also write-

$$
\oint\left(\nabla \rho_{\phi}\right) d \vec{A}=G_{x}^{\prime} E
$$

$$
\text { ( } d \vec{A} \text { Area element) }
$$

Or by using divergence theorem-

$$
\left(\nabla \rho_{\phi}\right) d \mathcal{V} \text { or } \int_{\mathcal{V}} \nabla\left(\nabla \rho_{\phi}\right) d \mathcal{V}=G_{x}^{\prime} E=\oint\left(\nabla \rho_{\phi}\right) d \vec{A}
$$

In this way we can also define these field equations or in terms of differential operators, we can define these things as-

$$
\int_{\mathcal{M}^{n}} \partial_{\mu} \partial_{v}\left(\rho_{\phi}\right) d^{n} x=G_{x} E
$$

Here is the volume element of the end dimensional scalar field density is around and dimensional geometrical bodies. For different dimensional bodies the effect of variation scalar field is different. As I mentioned earlier in my former paper to that minor singularities (like black holes etc) have different kind of scalar field around themselves. So, these minor singularities have different kind of scalar field density variation force nature not like inverse square law around themselves. Show, inverse square Law holds in a special case, note two the whole Universe. For flat spacetime (zero variation in the scalar field density or there does not exist any quantity $(\psi)$ ) we get equation [273] as-

$$
\int_{\mathcal{V}} \nabla^{2} \rho_{\phi} d \mathcal{V}=G_{x}^{\prime} \psi \phi=0 \quad \text { for } \psi=0 \text { flat spacetime }
$$

So, this follows Laplace equation [38], as-

$$
\nabla^{2} \rho_{\phi}=0
$$

So, this is the flat spacetime condition holds in end dimensional space time. This holds in quantum field theory (on microscopic scale) or General Relativity and special relativity or macroscopic scale also. In general we can write equation (273) curved space time by existence of quantity $(\psi)$ as for constant $\nabla^{2} \rho_{\phi}$ as-

$$
\nabla^{2} \rho_{\phi} \int_{\mathcal{V}} d \mathcal{V}=G_{x}^{\prime} E
$$

Or

$$
\nabla^{2} \rho_{\phi} \cdot \mathcal{V}=G_{x}^{\prime} E
$$

$$
\nabla^{2} \rho_{\phi}=G_{x}^{\prime} \frac{E}{\mathcal{v}}
$$

Now as we know $\frac{E}{v}=\mathcal{E}$ (energy density) from my former paper[6]. So by putting $\mathcal{E}$ value in equation (279), we get-

$$
\nabla^{2} \rho_{\phi}=G_{x}^{\prime} \mathcal{E}
$$

So this follows the position equation [31], we can also write this particular equation as-

$$
\nabla^{2} \rho_{\phi}=G_{x}^{\prime} \psi \rho_{\phi}
$$




$$
\frac{1}{\rho_{\phi}} \nabla^{2} \rho_{\phi}=G_{x}^{\prime} \psi
$$

\section{GENERALIZED QUANTUM MECHANICS}

Now I am defining rules of Quantum Mechanics in terms of same theoretical perspectives. Let's start with ground state according to N-Time Inflationary Model of Universe a ground state is which position of a body that defines the basic constants of the body in different eras of Universe". The ground states for same Central Systems are same in a particular epoch but different in different parts of Universe due to principal of Central System Relativity. If we know about ground state of a system then, we can define other states of it.

\subsection{Generalized Uncertainty Principle}

Now I intend to obtain Heisenberg Uncertainty relation in terms of my Generalized Uncertainty Principle or Generalized Correspondence Principle mathematically.

By Werner Heisenberg [14]-

$$
\Delta \mathrm{x} . \Delta \mathrm{p} \geq \frac{\mathrm{h}}{2}
$$

Now in unique way we can write it for and N-Central System as-

$$
\Delta \mathrm{s} . \Delta \mathrm{F} \geq \mathrm{c} \hbar_{x}
$$

Or in more simplified way

$$
\Delta \Phi \Delta \psi \geq c^{\prime} \hbar_{x}
$$

Here is some constant. So, this is the mathematical statement of Generalized Uncertainty Principle which is a manifestation of $\phi-\psi$ transformation.

Or this can be written by using $\Delta \psi=\alpha \Delta \Phi$ as-

$$
(\Delta \psi)^{2} \geq c^{\prime} \alpha \hbar_{x}
$$

Or

$$
(\Delta \Phi)^{2} \geq \frac{c^{\prime}}{\alpha} \hbar_{x}
$$

Now by solving for the two equation for time- energy Uncertainty-

$$
\Delta \tau . \Delta E \geq c \hbar_{x}
$$

$$
\because \quad \Delta \tau=\frac{\Delta \Phi}{\Gamma(\Phi) \Phi} \text { Or } \Delta E=\Delta(\Phi \psi)=\psi \Delta \Phi+\Phi \Delta \psi
$$

Now by putting both values in equation (288), we get-

$$
\frac{\Delta \Phi}{\Gamma(\Phi) \Phi}(\psi \Delta \Phi+\Phi \Delta \psi) \geq c \hbar_{x}
$$

Now by solving further, we get-

$$
\psi(\Delta \Phi)^{2}+\Phi \alpha(\Delta \Phi)^{2} \geq c \Gamma(\Phi) \Phi \hbar_{x}
$$

Or

$$
(\Delta \Phi)^{2}(\psi+\Phi \alpha) \geq c \hbar_{x} \Gamma(\Phi) \Phi
$$

Or

$$
\left.(\Delta \Phi)^{2}\right) \geq c \hbar_{x}\left(\frac{\Gamma(\Phi) \Phi}{\psi+\Phi \alpha}\right)
$$

Now by comparing both equations (292) and (287), we get-

$$
c \cdot \frac{\Gamma(\Phi) \Phi}{\psi+\Phi \alpha}=\frac{c^{\prime}}{\alpha}
$$

Or

$$
c^{\prime}=\frac{c \cdot \alpha \Gamma(\Phi) \Phi}{(\psi+\alpha \Phi)}
$$

By putting value in equation (285), we get-

$$
\Delta \Phi \Delta \psi \geq \frac{\Gamma(\Phi) \Phi \alpha}{(\psi+\alpha \Phi)} c \hbar_{x}
$$

So, this Uncertainty relation is unique in all Central Systems for observer O' to O" in Principle of Central System Relativity. 
For atomic Central Systems $e=\frac{1}{2}$ or $\hbar_{x}=\hbar=$ Planck constant-

$$
\Delta \Phi \Delta \psi \geq \frac{\Gamma(\Phi) \Phi \alpha}{(\psi+\alpha \Phi)} \frac{1}{2} \hbar_{x}
$$

Or we can write it as-

$$
\begin{gathered}
\Delta \Phi \Delta \psi \frac{\psi_{t}}{\psi^{\prime} \Gamma(\Phi)} \geq \frac{1}{2} \hbar \\
\left\{\because \quad \psi_{t}=\psi+\alpha \Phi, \psi^{\prime}=\Phi \alpha\right\}
\end{gathered}
$$

We can also write above expression as-

$$
\Delta \Phi \Delta \psi \frac{1}{\Gamma(\Phi)}\left(1+\frac{\psi}{\psi^{\prime}}\right) \geq \frac{\hbar}{2}
$$

Now by comparing this particular relation with Heisenberg's Uncertainty relation in equation (283), we get-

$$
\Delta x \Delta p=\Delta \Phi \Delta \psi \frac{1}{\Gamma(\Phi)}\left(1+\frac{\psi}{\psi^{\prime}}\right)
$$

Now while putting $\Delta x=\Delta s$ and $\Delta p=\Delta F$ in my theoretical perspective, we get-

$$
\Delta s . \Delta F=\Delta \Phi \Delta \psi \frac{1}{\Gamma(\Phi)}\left(1+\frac{\psi}{\psi^{\prime}}\right)
$$

This particular equation is valid in all Central Systems period now by putting value from equation (255), we get-

$$
\Delta s\left(\frac{\partial F}{\partial \tau} \Delta \tau+\frac{\partial F}{\partial \phi} \Delta \Phi\right)=\Delta \Phi \Delta \psi \frac{1}{\Gamma(\Phi)}\left(1+\frac{\psi}{\psi^{\prime}}\right)
$$

Now by putting, $\Delta \tau=\frac{\Delta \Phi}{\Gamma(\Phi) \Phi}$ we get-

$$
\Delta s\left(\frac{\partial F}{\partial \tau} \cdot \frac{\Delta \Phi}{\Gamma(\Phi) \Phi}+\frac{\partial F}{\partial \phi} \Delta \Phi\right)=\Delta \Phi \Delta \psi \frac{1}{\Gamma(\Phi)}\left(1+\frac{\psi}{\psi^{\prime}}\right)
$$

Now by removing $\Delta \Phi$ terms both sides and by some manipulations, we get-

$$
\Delta s\left(\frac{\partial F}{\partial \tau}+\frac{\partial F}{\partial \phi} \Gamma(\Phi) \Phi\right)=\Delta \psi \cdot \frac{\Phi}{\alpha \Phi}\left(\psi_{t}\right)
$$

Or

$$
\Delta s\left(\frac{\partial F}{\partial \tau}+\Gamma(\Phi) \Phi \cdot \frac{\partial F}{\partial \phi}\right)=\Delta \psi \cdot\left(\frac{\psi_{t}}{\alpha}\right)
$$

$$
\begin{gathered}
\left\{\because \psi_{t}=\psi+\psi^{\prime}=\psi+\alpha \Phi \text { or } \psi_{t}^{\prime}=\left(\psi+\psi^{\prime}\right)^{\prime}=\psi^{\prime}+\psi\right. \\
\left.=\alpha \Phi=\psi_{t}\right\} \\
\left\{\because \Phi_{t}=\Phi+\Phi^{\prime}=\Phi+\frac{\psi}{\alpha}=\frac{\alpha \Phi+\psi}{\alpha}=\frac{\psi+\psi^{\prime}}{\alpha}=\frac{\psi_{t}}{\alpha}\right\}
\end{gathered}
$$

Now by putting these relations, we get equation (304) as-

$$
\left(\frac{\partial F}{\partial \tau}+\frac{\partial F}{\partial \phi} \Gamma(\Phi) \Phi\right)=\frac{\Delta \psi}{\Delta s} . \Phi_{t}
$$

We can also write similar equation as-

$$
\left(\frac{\partial F}{\partial \tau}+\frac{\partial F}{\partial \phi} \Gamma(\Phi) \Phi\right)=\psi_{t} \cdot \frac{\Delta \Phi}{\Delta s}
$$

The right hand side is some form of force $(\delta)$. Here by putting right hand side as $\delta_{n}$, we get both equations (309) and (306) as-

$$
\delta_{n}=\frac{\Delta \psi}{\Delta s} \cdot \Phi_{t}=\psi_{t} \cdot \frac{\Delta \Phi}{\Delta s}
$$

For small variations we can also write it as-

$$
\delta_{n}=\psi_{t} \cdot \frac{d \Phi}{d s}=\frac{d \psi}{d s} . \Phi_{t}
$$

Here $s$ is the parameter of space time. Newton's second law 32 does not hold for $\phi-\psi$ transformations. Now a different kind of energy term comes out in form of $\psi_{t}$ and $\Phi_{t}$ as-

$$
E_{t}=\psi_{t} \Phi_{t}=\frac{\psi_{t}^{2}}{\alpha}=\alpha \Phi_{t}^{2}
$$

This is also a fundamental form of energy and this is maximum energy for a body possible. 


$$
\begin{aligned}
E_{t}=\left(\psi+\psi^{\prime}\right)(\Phi & \left.+\Phi^{\prime}\right)=\psi \Phi+\psi^{\prime} \phi^{\prime}+\psi^{\prime} \phi+\phi^{\prime} \psi \\
& =\psi \Phi+\alpha \phi \psi / \alpha+\psi \phi^{2}+\psi^{2} / \alpha \\
& =2 \psi \phi+\frac{1}{\alpha}\left(\psi^{2}+\psi^{2} \phi^{2}\right) \\
& =2 E+\frac{1}{\alpha}\left(\psi^{2}+\psi^{2} \phi^{2}\right)
\end{aligned}
$$

So, from here we can conclude that this energy terms is almost four times greater than $\psi \phi=E$.

\subsection{Conservation of Energy}

Now according to my theoretical perspective as the velocity increases of body its converged quantity also increasing. So, what about energy conservation in the case? As we know-

$$
E=\psi \phi=E_{c}+E_{p}=\psi_{p} \phi+\alpha \phi \Delta \phi
$$

Now if-

$$
v_{p}^{2} \propto \Delta \phi
$$

Then, as $v_{p} \uparrow, E_{c} \uparrow$ or the quantity transformed from $\psi_{p}$ or $\left(\psi_{p}\right)_{t}$ (Transformable Perfection Quantity). So, as $\Delta \phi \uparrow$ $\psi_{p} \downarrow$ or $E_{c} \uparrow E_{p} \downarrow$ or sum of these are always same. So, we get-

$$
E_{1}=\psi_{p_{1}} \phi_{1}+\alpha \phi_{1} \Delta \phi_{1}
$$

$$
E_{2}=\psi_{p_{2}} \phi_{2}+\alpha \phi_{2} \Delta \phi_{2}
$$

$$
\text { If } \Delta \phi_{1}=\Delta \phi_{2}+\Delta \phi \text { or } \psi_{p_{1}}=\psi_{p_{2}}-\Delta \psi_{p}
$$

For conservation of energy-

$$
E_{1}=E_{2}
$$

$$
\phi_{1}\left[\psi_{p_{1}}+\alpha \Delta \phi_{1}\right]=\phi_{2}\left[\psi_{p_{2}}+\alpha \Delta \phi_{2}\right]
$$

$$
\phi_{1}\left[\psi_{p_{2}}-\Delta \psi_{p}+\alpha \Delta \phi_{2}+\alpha \Delta \phi\right]=\phi_{2}\left[\psi_{p_{2}}+\alpha \Delta \phi_{2}\right]
$$

If $\phi_{1}=\phi_{2}$ (or there is no change in the unique quantity of body's scalar field which is usually non-transformable), then-

$$
-\Delta \psi_{p}+\alpha \Delta \phi=0
$$

Therefore-

$$
\Delta \psi_{p}=\alpha \Delta \phi
$$

So, Conservation of energy holds during $\phi-\psi$ transformation also.

As we know $\psi \geq \alpha \phi$ form my former paper [6].

$$
\psi^{\prime} \phi+\phi^{\prime} \psi \cong 2 E
$$

$$
\left\{\because E=\psi^{\prime} \phi^{\prime}=\psi \phi\right\}
$$$$
\psi^{\prime} \phi+\phi^{\prime} \psi \cong \psi^{\prime} \phi^{\prime}+\psi^{\prime} \phi^{\prime}
$$

$$
\psi^{\prime}\left(\phi-\phi^{\prime}\right) \cong \phi^{\prime}\left(\psi^{\prime}-\psi\right)
$$

$$
\phi\left(\psi^{\prime}-\psi\right) \cong \phi^{\prime}\left(\psi^{\prime}-\psi\right)
$$

Now by removing similar terms-

$$
\phi \cong \phi^{\prime}
$$

By multiplying both sides with, we get-

$$
\alpha \phi \cong \alpha \phi^{\prime}
$$

$$
\alpha \phi \leq \psi
$$

Or

$$
\psi^{\prime} \phi+\phi^{\prime} \psi \leq 2 E
$$

Now by adding both sides to the inequality, we get-

$$
2 E+\psi^{\prime} \phi+\phi^{\prime} \psi \leq 4 E
$$


Left hand side is equal to equation (310) or $E_{t}$ we get limit of maximum energy as-

$$
E_{t} \leq 4 E
$$

So, a particular body cannot exceed this energy from. Now by taking the variation both sides in equation (328), we get-

$$
\Delta E \geq \frac{1}{2} \Delta\left(\psi^{\prime} \phi+\phi^{\prime} \psi\right)
$$

$$
\psi \Delta \Phi+\Delta \psi \Phi \geq \frac{1}{2}\left(\psi^{\prime} \Delta \Phi+\Delta \psi^{\prime} \Phi+\phi^{\prime} \Delta \psi+\Delta \phi^{\prime} \psi\right)
$$

Now by putting $\Delta \psi=\alpha \Delta \Phi$ and $\psi^{\prime}=\alpha \Phi$, we get-

$$
\begin{aligned}
\psi \Delta \Phi+\alpha \Phi \Delta \Phi \geq & \frac{1}{2}\left[\alpha \Phi \Delta \Phi+\alpha \Phi \Delta \Phi+\Phi^{2} \Delta \alpha+\alpha \Phi^{\prime} \Delta \Phi\right. \\
& \left.+\psi\left(\frac{\alpha \Delta \psi-\psi \Delta \alpha}{\alpha^{2}}\right)\right]
\end{aligned}
$$

Now by eliminating similar terms and by solving further, we get-

$$
\psi \Delta \Phi \geq \frac{1}{2}\left(\Phi^{2} \Delta \alpha+\psi \Delta \Phi-\frac{\psi^{2}}{\alpha^{2}} \cdot \Delta \alpha+\frac{\psi}{\alpha} \cdot \Delta \psi\right)
$$

Now by putting $\Delta \psi=\alpha \Delta \Phi$, we get-

$$
\psi \Delta \Phi \geq \frac{1}{2}\left(\frac{\Delta \alpha}{\alpha^{2}}\left(\alpha^{2} \Phi^{2}-\psi^{2}\right)+\psi \Delta \Phi+\psi \Delta \Phi\right)
$$

Now by eliminating similar terms, we get-

$$
\frac{\Delta \alpha}{2 \alpha^{2}}(\alpha \Phi-\psi)(\alpha \Phi+\psi) \leq 0
$$

$\alpha^{2}$ Is positive and $\Delta \alpha+\psi$ is also positive. So from here we find something related to my former terms, as-

$$
\Delta \alpha(\alpha \Phi-\psi) \leq 0
$$

Or

$$
\Delta \alpha(\alpha \Phi+\psi) \leq 0
$$

Here two conditions are applied-

(1) When $\Delta \alpha=+v e$ or $\Delta \alpha \geq 0$, then-

$$
\psi-\alpha \phi \geq 0
$$

Or

$$
\psi \geq \alpha \phi
$$

When $\Delta \alpha=-v e$ or $\Delta \alpha \leq 0$, then-

$$
\psi-\alpha \phi \leq 0
$$

Or

$$
\psi \leq \alpha \phi
$$

Now by taking variations of both equation (339) and (340), we get-

$$
\Delta \psi \geq \Delta \alpha . \phi+\alpha \Delta \phi
$$

For inflations [5] and for inflations we know that-

$$
|\alpha \Delta \phi|=-\alpha \Delta \phi
$$

So, we get-

$$
\Delta \psi+\alpha \Delta \phi \geq \phi \Delta \alpha_{i}
$$

Or

$$
\Delta \alpha_{i} \leq \frac{1}{\phi}(|\Delta \psi|+\alpha|\Delta \phi|)
$$

$$
\because|\Delta \psi|=\alpha|\Delta \phi|
$$

Therefore

$$
\Delta \alpha_{i} \leq \frac{2|\Delta \psi|}{\phi}
$$

But for equation (340), we know that- 
$\Delta \psi \leq \alpha \Delta \phi+\phi \Delta \alpha_{d}$ for $\Delta \alpha \leq 0$

Or $\Delta \alpha \leq 0$ for deflations [5] and for deflation we know that-

$$
\alpha|\Delta \phi|=\alpha \Delta \phi=|\Delta \psi|
$$

So, we get-

$$
\phi \Delta \alpha_{d}+\alpha \Delta \phi \leq|\Delta \psi|
$$

$$
\phi \Delta \alpha_{d} \leq 0
$$

So, $\Delta \alpha=-v e$ for deflations or in other words we can say conversion rate decreases during deflations. At critical epoch $\Delta \alpha=0$ or there is zero variation in conversion rate. Here critical epoch is referred as the epoch of minor singularities and new formations (totally imperfect bodies). So, this whole thing can be represented in time N-Time Inflationary Model of Universe as-

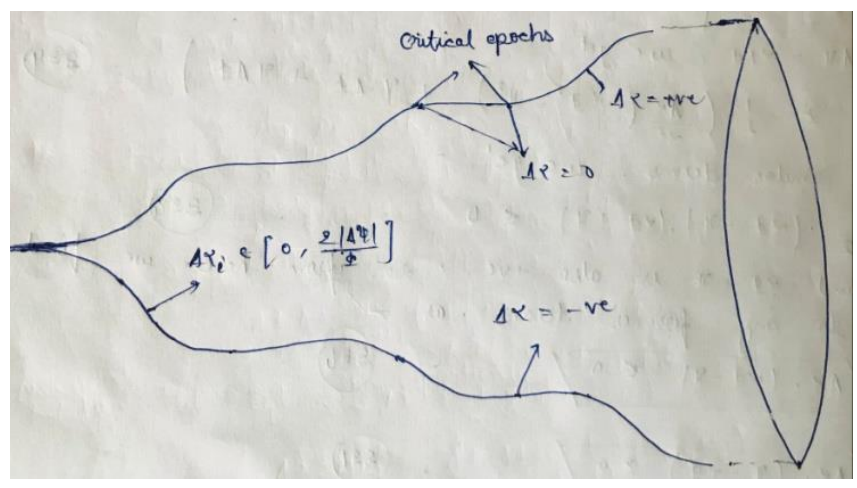

FIG. 10 Geometrical Representation of Critical Epochs in NTime Inflationary Model of Universe

So, we can clearly see from above representation that at critical epochs phase transitions occurs in steady states $(\Delta \alpha=$ $0)$.

\subsection{Generalized Schrödinger Equation}

Now I intend to define Schrödinger's wave equation [16] in terms of this theoretical perspective. This equation is totally dependent upon the Wave-Particle Duality [13] and Hamilton Jacobi equation [17], as I defined the dual nature of functions in my $5^{\text {th }}$ paper [5].

$$
\begin{array}{r}
\phi=\phi_{1}+i \phi_{2} \\
\psi=\psi_{1}+i \psi_{2}
\end{array}
$$

$$
\tau=\tau_{1}+i \tau_{2}
$$

Or

$$
\psi=|\psi| e^{i \theta_{\psi}}, \phi=|\phi| e^{i \theta_{\phi}} \text { or } \tau=|\tau| e^{i \theta_{\tau}}
$$

Here real parts represent attractive nature of scalar field, quantity or time and imaginary parts represent repulsive nature of these physical quantities.

$$
\theta_{\psi}=\tan ^{-1} D_{\psi}, \theta_{\phi}=\tan ^{-1} D_{\phi} \text { or } \theta_{\tau}=\tan ^{-1} D_{\tau}
$$

Here $D_{x}$ is the duality factor of particular physical quantity.

$$
E=\phi \psi=|\phi||\psi| e^{i \theta_{\psi}} e^{i \theta_{\phi}}
$$

Now by differentiating above equation with respect to $\tau$ (time), we get-

$$
\begin{aligned}
\frac{\partial E}{\partial \tau}=\left(\psi \frac{\partial \phi}{\partial \tau}+\phi\right. & \left.\frac{\partial \psi}{\partial \tau}\right) e^{i\left(\theta_{\psi}+\theta_{\phi}-\theta_{\tau}\right)} \\
& +i\left(\frac{\partial \theta_{\psi}}{\partial \tau}+\frac{\partial \theta_{\phi}}{\partial \tau}-\frac{\partial \theta_{\tau}}{\partial \tau}\right) \phi \psi e^{i\left(\theta_{\psi}+\theta_{\phi}-\theta_{\tau}\right)}
\end{aligned}
$$

Here we have taken $\psi=|\psi| \phi=|\phi|$ and $\tau=|\tau|$.

So, we can write above equation by some manipulations, as-

$$
\frac{\partial E}{\partial \tau}=\left(\psi \frac{\partial \phi}{\partial \tau}+\phi \frac{\partial \psi}{\partial \tau}\right) e^{i \theta}+i\left(\frac{\partial \theta}{\partial \tau}\right) \phi \psi e^{i \theta}
$$

$$
\text { Here } \theta=\theta_{\psi}+\theta_{\phi}-\theta_{\tau}
$$

For conserved energy systems-

$$
\left(\psi \frac{\partial \phi}{\partial \tau}+\phi \frac{\partial \psi}{\partial \tau}\right)=-i E \frac{\partial \theta}{\partial \tau}
$$

Or we get-

$$
\left(\psi \frac{\partial \phi}{\partial \tau}+\phi \frac{\partial \psi}{\partial \tau}\right)+i E \frac{\partial \theta}{\partial \tau}=0
$$

Or we can write it by multiplying by $i$ as- 


$$
i \psi \frac{\partial \phi}{\partial \tau}+i \phi \frac{\partial \psi}{\partial \tau}=E \frac{\partial \theta}{\partial \tau}
$$

Here $\frac{\partial \theta}{\partial \tau}$ shows the wave nature of bodies in Universal Mechanics.

Now from my $5^{\text {th }}$ paper [5], we know from equation (61), that

$$
F=i \psi \phi_{\text {covered }} \cdot \frac{\partial \theta}{\partial \tau}+\frac{\psi \phi_{\text {covered }}}{\left(\phi_{1}^{2}+\phi_{2}^{2}\right)}\left(\phi_{1} \frac{\partial \phi_{1}}{\partial \tau}+\phi_{2} \frac{\partial \phi_{2}}{\partial \tau}\right)
$$

$$
\begin{gathered}
\vec{F}=\psi \frac{\partial \vec{s}}{\partial \tau}, \quad \frac{\partial s}{\partial \tau}=\phi_{\text {covered }} \\
F=\psi \cdot e^{i \theta_{\psi}} \frac{\partial s}{\partial \tau} \cdot e^{i\left(\theta_{\phi}-\theta_{\tau}\right)}=\psi \frac{\partial s}{\partial \tau} e^{i \theta}+i \psi s\left(\frac{\partial \theta_{\phi}}{\partial \tau}-\frac{\partial \theta_{\tau}}{\partial \tau}\right) e^{i \theta}
\end{gathered}
$$

Here space has same nature as scalar field because it is spacetime or time is the flow of scalar field.

$$
\frac{\partial s}{\partial \tau}=\phi_{\text {covered }}=v_{p}+k_{\phi} \cdot \mathrm{F}\left(\Phi_{B}\right)+k_{\phi}^{\prime} \cdot \mathrm{F}\left(\Phi_{u}\right)
$$

Now by putting this value in above equation, we get-

$$
F=\psi \cdot e^{i \theta}\left\{v_{p}+k_{\phi} \cdot \mathrm{F}\left(\Phi_{B}\right)+k_{\phi}^{\prime} \cdot \mathrm{F}\left(\Phi_{u}\right)+i s\left(\frac{\partial\left(\theta-\theta_{\psi}\right)}{\partial \tau}\right)\right\}
$$

By ignoring these flow induced velocities for observers on earth, we get-

$$
F=\psi \cdot e^{i \theta}\left\{v_{p}+i s\left(\frac{\partial \theta^{\prime}}{\partial \tau}\right)\right\}
$$

$$
\left\{\text { Here } \theta^{\prime}=\theta-\theta_{\psi}=\theta_{\phi}-\theta_{\tau}\right\}
$$

We can also define kinetic energy $E_{k}$ as-

$$
E_{k}=\int_{0}^{v_{p}} F \cdot d v_{p}=\int_{0}^{v_{p}} \psi v_{p} \cdot d v_{p}=\frac{1}{2} \psi v_{p}^{2}
$$

But in universal case-

$$
E_{k}=\int_{0}^{v_{p}^{\prime}} \psi v_{p}^{\prime} \cdot d v_{p}^{\prime}=\frac{1}{2} \psi\left(v_{p}^{\prime}\right)^{2}
$$

Now by putting the value of $v_{p}^{\prime}$, we get-

$$
E_{k}=\frac{1}{2} \psi\left(v_{p}+k_{\phi} \cdot \mathrm{F}\left(\Phi_{B}\right)+k_{\phi}^{\prime} \cdot \mathrm{F}\left(\Phi_{u}\right)\right)^{2}
$$

$$
E_{k}=\frac{1}{2} \psi\left(v_{p}+v_{B}+v_{u}\right)^{2}
$$

For broken part kinetic energy-

$$
\left(E_{k}\right)_{b}=\frac{1}{2} \psi_{b}\left(v_{p}\right)_{c}^{2}
$$

As we know $\psi_{b}=\alpha \Delta \phi_{b}$ for broken part.

$$
E_{b}=\phi_{b} \psi_{b}=\alpha \phi_{b} \Delta \phi_{b}
$$

By special relativity-

$$
E=m c^{2}=\psi_{b}\left(v_{p}\right)_{c}^{2}=2\left(E_{k}\right)_{b}
$$

$$
E=h v=E_{b}=\phi_{b} \psi_{b}
$$

Now by comparison of both equations (369) and (370), we get-

$$
\phi_{b}=\left(v_{p}\right)_{c}^{2}
$$

Or

$$
h_{x}=\frac{\phi_{b} \psi_{b}}{v_{x}}
$$

Now from here very classical point comes out from universal relativistic point of view. If two observers differently observing broken parts, then-

$$
\begin{aligned}
\phi_{b}^{\prime}=\left(v_{p}^{\prime}\right)^{2}=[ & \left.\left(v_{p}\right)_{c}+v_{B}+v_{u}\right]^{2} \\
& =\left(v_{p}\right)_{c}^{2}+v_{B}^{2}+v_{u}^{2}+2\left(v_{p}\right)_{c} v_{B} \\
& +2\left(v_{p}\right)_{c} v_{u}+2 v_{B} v_{u}
\end{aligned}
$$


From here by putting value of from equation (371), we get-

$$
\phi_{b}^{\prime}-\phi_{b}=v_{B}^{2}+v_{u}^{2}+2\left(v_{p}\right)_{c} v_{B}+2\left(v_{p}\right)_{c} v_{u}+2 v_{B} v_{u}
$$

So, if broken part is influenced by universal dynamics with and somebody reason for gravitational bending with $v_{B}$, then two observers from different epochs will observe the energy of same broken part differently by Principle of Universal Relativity. Energy difference observed can be calculated by multiplying equation (374) by as-

$$
\begin{aligned}
\psi_{b} \phi_{b}^{\prime}-\psi_{b} \phi_{b}= & \psi_{b}\left(v_{B}^{2}+v_{u}^{2}+2\left(v_{p}\right)_{c} v_{B}+2\left(v_{p}\right)_{c} v_{u}\right. \\
& \left.+2 v_{B} v_{u}\right)=\Delta E_{b}^{R}
\end{aligned}
$$

Now by putting value of $h_{x}$ in Generalized Uncertainty relations in equation (295) from equation (372), we get-

$$
\Delta \Phi \Delta \psi \geq \frac{\Gamma(\Phi) \Phi \alpha}{(\psi+\alpha \Phi)} c \hbar_{x}=\frac{\Gamma(\Phi) \alpha \Phi}{(\psi+\alpha \Phi)} \frac{c}{n \pi} \frac{\phi_{b} \psi_{b}}{v_{x}}
$$

If two different observers obtaining different $E_{b}$, then-

$$
h_{x}^{\prime}=\frac{E_{b}^{\prime}}{v_{x}} \text { or } h_{x}=\frac{E_{b}}{v_{x}}
$$

Or their observation for fundamental Uncertainty constant will be different-

$$
\Delta h_{x}^{R}=\frac{\Delta E_{b}^{R}}{v_{x}}
$$

Here in equation (376) Observers observed broken parts of same body independently. For broken parts this Uncertainty relation can be written as-

$$
\begin{gathered}
\Delta \phi_{b} \Delta \psi_{b} \geq \frac{\alpha \phi_{b} \cdot \Gamma\left(\phi_{b}\right)}{\left(\psi_{b}+\alpha \phi_{b}\right)} \frac{c}{n \pi} \frac{\phi_{b} \psi_{b}}{v_{x}} \\
\Delta \psi_{b}=\psi_{b} \text { or } \Delta \phi_{b}=\frac{\psi_{b}}{\alpha}
\end{gathered}
$$

By putting these relations in above equation, we get-

$$
\psi_{b} \frac{\psi_{b}}{\alpha} \geq \frac{\alpha \phi_{b}}{\left(\psi_{b}+\alpha \phi_{b}\right)} \frac{c \cdot \Gamma\left(\phi_{b}\right)}{n \pi} \frac{\phi_{b} \psi_{b}}{v_{x}}
$$

Now by some manipulations, we get

$$
v_{x} \geq \frac{\phi_{b}}{\psi_{b}} \frac{\alpha^{2} \phi_{b}}{\left(\psi_{b}+\alpha \phi_{b}\right)} \frac{c \cdot \Gamma\left(\phi_{b}\right)}{n \pi}
$$

For atomic Central Systems or broken parts of atomic bodies (electron, proton or neutrons) or boson or broken parts of light (photons) we get-

$$
\begin{gathered}
\frac{c}{n \pi}=\frac{1}{4 \pi}, c=\frac{1}{2}, \hbar=\frac{h}{2 \pi} \\
v_{a} \geq \frac{1}{4 \pi} \frac{\alpha^{2} \phi_{b}^{2}}{\left(\psi_{b}^{2}+\alpha^{2} \phi_{b} \Delta \phi_{b}\right)} \cdot \Gamma\left(\phi_{b}\right)
\end{gathered}
$$

Now I am putting special relativistic mass energy equivalence is a limit to quantity-spacetime equivalence $E=\phi \psi$ or from Einstein's point of view quantity of body (or mass) can only be transformed into broken parts of atoms (or photons) only, but when we examine planets transformed in their broken parts (or asteroids). Now we can represent this thing geometrically as-

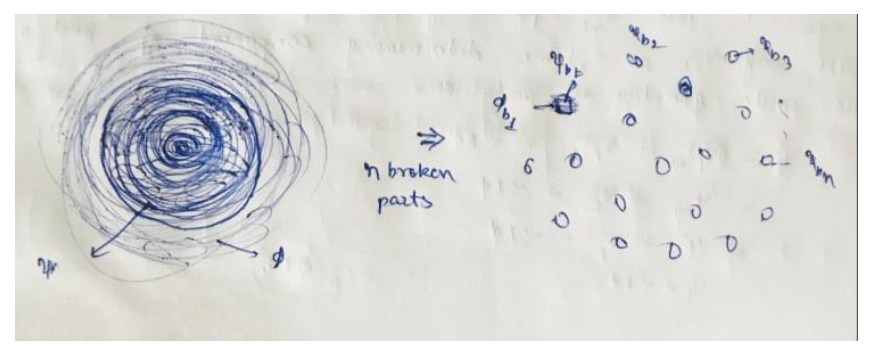

FIG. 11 Geometrical Representation of Conversion of Body in Broken Parts defined by Albert Einstein

We can write above geometrical expression mathematically, as-

$$
E=\phi \psi=\sum_{i=0}^{n}\left(\phi_{b} \psi_{b}\right)_{i}=\psi\left(v_{p}\right)_{c}^{2} \cong m c^{2}
$$

For same quantity of each broken part, we get-

$$
n \phi_{b} \psi_{b}=n h v=E=\phi \psi
$$

But according to $\phi-\psi$ transformation quantity transforms into scalar field will give the maximum energy note the quantity transforms in broken parts. So Einstein's energy mass equivalence is brilliant into itself but not a proper relation to energy $\phi-\psi$ transformation from quantity because it is valid only for broken parts. Now I am defining another beautiful 
fact from here that "if a body have quantity and it is formed out by universal quantity $\left(\psi_{u}\right)$, then the initial quantity broken from $\psi_{u}$ will never transform in scalar field in any situation, at least not in minor singularities also", but according to Hawking's time definition black hole totally transforms and become invisible after blasting. So, as I defined this fact is not properly right because black holes or minor singularities and minor singularities only change the flow of time, note the starting of time because after minor singularities also some quantity remains non-transformable $\left(\psi_{n t}\right)$, which manipulates time (as I defined earlier quantity manipulates time). So, if some quantity is not destructible, then time only starts at major singularity. We can represent the non transformable quantity as-

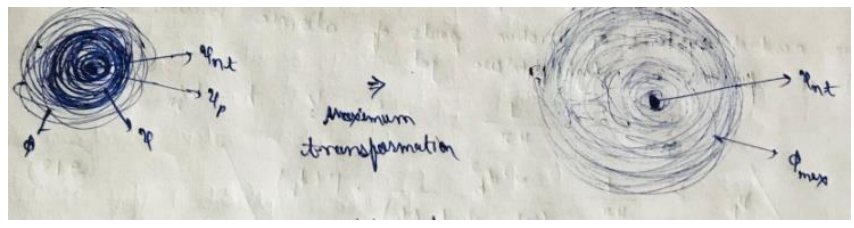

FIG. 12 Geometrical Representation of Quantity of Body in Scalar Field defined by me

We can write total quantity $\psi_{t}$ of body as-

$$
\begin{aligned}
\psi_{t}=\psi+\alpha \phi= & \psi_{p}+\alpha \Delta \phi+\alpha \phi \\
& =\left(\psi_{p}\right)_{t}+\left(\psi_{p}\right)_{n t}+\alpha \Delta \phi+\alpha \phi \\
& =\left(\psi_{p}\right)_{t}+\psi_{n t}+\alpha \Delta \phi+\alpha \phi \\
& =\psi_{n t}+\alpha \phi_{\max }
\end{aligned}
$$

Here $\left(\psi_{p}\right)_{t}+\alpha \Delta \phi$ is usually transformable quantity in body. At the maximum $\phi-\psi$ transformation body intend towards new formation or inflation age. This $\psi_{n t}$ part only transforms at major singularity. We can also say as time determining component of body. We can also write quantity of body as-

$$
\begin{aligned}
\psi=\left(\psi_{p}\right)_{t}+\psi_{n t} & +\alpha \Delta \phi=\psi_{n t}+\psi_{p}^{\prime}+\alpha \Delta \phi \\
= & \psi^{\circ}+\psi_{p}^{\prime}+\alpha \Delta \phi=\psi_{p}+\alpha \Delta \phi
\end{aligned}
$$

From here simply we can write-

$$
\psi_{p}=\psi^{\circ}+\psi_{p}^{\prime}
$$

Now for energy, we get-

$$
\begin{gathered}
E=\phi \psi=\left(\psi^{\circ}+\psi_{p}^{\prime}+\alpha \Delta \phi\right) \phi=\psi^{\circ} \phi+\psi_{p}^{\prime} \phi+\alpha \phi \Delta \phi \\
=E^{\circ}+E_{p}^{\prime}+E_{c}=E^{\circ}+E_{p}+E_{c}
\end{gathered}
$$

$$
\operatorname{Here} \psi_{p}^{\prime} \phi \cong \psi_{p} \phi \Rightarrow E_{p}^{\prime} \cong E_{p}, \because \psi_{p} \gg \psi^{\circ}
$$

Here we have used $\psi_{p} \cong \psi_{p}^{\prime}$ for shake of simplicity.

Usually is a general form of zero point energy defined in Quantum Mechanics. So, there we get both quantum mechanical and special relativistic energy from same equation. Here $\psi_{p}^{\prime}$ is also easily non-transformable quantity but in case of minor singularities this quantity transforms in scalar field.

Now let's come to equation (358) and Schrödinger's equation [17]-

$$
i \psi \frac{\partial \phi}{\partial \tau}+i \phi \frac{\partial \psi}{\partial \tau}=E \frac{\partial \theta}{\partial \tau}
$$

Or we can also write this equation, as-

$$
i \frac{\partial(\phi \psi)}{\partial \tau}=E \frac{\partial \theta}{\partial \tau}
$$

Or

$$
i \frac{\partial E}{\partial \tau}=E \frac{\partial \theta}{\partial \tau}
$$

Now by multiplying both sides with $i$, we get-

$$
-\frac{\partial E}{\partial \tau}=i E \frac{\partial \theta}{\partial \tau}
$$

$$
i E \frac{\partial \theta}{\partial \tau}+\frac{\partial E}{\partial \tau}=0
$$

For $\phi-\psi$ transformations, this equation is valid.

Now let's form a wave function $\mathcal{P}(s, F) \equiv \mathcal{P}(x, p)=\mathcal{P}(\tau, E)$ for observers in Central System Relativistic Case.

$$
\mathcal{P}(\tau, s)=A e^{\frac{i}{\hbar_{x}}(F . s-E . \tau)}
$$

Here is the amplitude of wave function.

This is valid in all type of Central Systems and $\hbar_{x}$ is the Generalized Uncertainty parameter. Now by differentiating this particular wave function with respect to time, we get- 


$$
\begin{aligned}
\frac{\partial \mathcal{P}(\tau, s)}{\partial \tau}=-i \cdot A & \cdot \frac{\partial}{\partial \tau}\left(\frac{E \cdot \tau}{\hbar_{x}}\right) \cdot e^{\frac{i}{\hbar_{x}}(F . s-E \cdot \tau)} \\
& +i A \cdot \frac{\partial}{\partial \tau}\left(\frac{F . s}{\hbar_{x}}\right) \cdot e^{\frac{i}{\hbar_{x}}(F . s-E . \tau)}
\end{aligned}
$$

Now by including Central System Relativity and Universal Relativity or $\phi-\psi$ transformation of spin in Universe with equation (378), we get-

$$
\frac{\partial \hbar_{x}}{\partial \tau} \neq 0
$$

Or by $\phi-\psi$ transformation-

$$
\frac{\partial E}{\partial \tau} \neq 0
$$

Now by Universal Relativity equation (378) can be written (for doing infinitesimal change) as-

$$
d h_{x}=\frac{d E_{b}}{v_{x}}
$$

Here $E_{b}$ is the energy of broken parts of same body and frequency remains same for broken part.

Only $\hbar_{x}$ can be same in case of-

$$
\hbar_{x}=\frac{E_{b}^{\prime}}{2 \pi v_{x}^{\prime}}=\frac{E_{b}}{2 \pi v_{x}}
$$

Here $\mathrm{n}=2$ for Spherical symmetries.

Hence

$$
\frac{\partial}{\partial \tau}\left(\frac{E \cdot \tau}{\hbar_{x}}\right)=\frac{E}{\hbar_{x}}+\frac{\tau}{\hbar_{x}} \frac{\partial E}{\partial \tau}+E \cdot \tau\left(-\frac{1}{\hbar_{x}^{2}}\right) \cdot \frac{\partial \hbar_{x}}{\partial \tau}
$$

Or for duality-

$$
\begin{aligned}
\frac{\partial}{\partial \tau}\left(\frac{E \cdot e^{i \theta^{\prime}} \cdot \tau \cdot e^{-i \theta_{\tau}} \cdot e^{i \theta_{\tau}}}{\hbar_{x}}\right) & \\
& =\frac{E \cdot e^{i \theta^{\prime}}}{\hbar_{x}}+\frac{\tau \cdot e^{i \theta^{\prime}}}{\hbar_{x}} \frac{\partial E}{\partial \tau} \\
& +E e^{i \theta^{\prime}} \cdot \tau\left(-\frac{1}{\hbar_{x}^{2}}\right) \cdot \frac{\partial \hbar_{x}}{\partial \tau}+i \frac{E \cdot \tau \cdot e^{i \theta^{\prime}}}{\hbar_{x}} \frac{\partial \theta^{\prime}}{\partial \tau}
\end{aligned}
$$

$$
\begin{gathered}
\left\{\text { Here } \theta^{\prime}=\theta_{\psi}+\theta_{\phi} \because E=\phi \psi=|\phi||\psi| e^{i\left(\theta_{\psi^{+}}+\theta_{\phi}\right)}\right. \\
\left.=|E| e^{i \theta^{\prime}}\right\}
\end{gathered}
$$

Now by putting equation (392) in above equation, we get-

$$
\begin{aligned}
\frac{\partial}{\partial \tau}\left(\frac{E \cdot \tau \cdot e^{i \theta^{\prime}}}{\hbar_{x}}\right)= & \left(\frac{E}{\hbar_{x}}-\frac{E \cdot \tau}{\hbar_{x}^{2}} \frac{\partial \hbar}{\partial \tau}\right) e^{i \theta^{\prime}}+\frac{\tau \cdot e^{i \theta^{\prime}}}{\hbar_{x}}\left(\frac{\partial E}{\partial \tau}+i E \frac{\partial \theta^{\prime}}{\partial \tau}\right) \\
& =\frac{E e^{i \theta^{\prime}}}{\hbar_{x}}\left(1-\frac{\tau}{\hbar_{x}} \frac{\partial \hbar_{x}}{\partial \tau}\right)
\end{aligned}
$$

$$
\left\{\because \frac{\partial E}{\partial \tau}+i E \frac{\partial \theta}{\partial \tau}=0 \text { or } \theta \approx \theta^{\prime} \text { then } \frac{\partial \theta}{\partial \tau} \approx \frac{\partial \theta^{\prime}}{\partial \tau}\right\}
$$

Now if $F$ is also independent of time, then-

$$
\frac{\partial}{\partial \tau}\left(\frac{F . s}{\hbar_{x}}\right)=\frac{s}{\hbar_{x}} \frac{\partial F}{\partial \tau}+\frac{F}{\hbar_{x}} \frac{\partial s}{\partial \tau}+F . s\left(-\frac{1}{\hbar_{x}^{2}}\right) \cdot \frac{\partial \hbar_{x}}{\partial \tau}
$$

Now in case of duality, we get-

$$
\begin{aligned}
\frac{\partial}{\partial \tau}\left(\frac{F . s . e^{i \theta^{\prime}}}{\hbar_{x}}\right)= & \frac{s . e^{i \theta^{\prime}}}{\hbar_{x}} \frac{\partial F}{\partial \tau}+\frac{F \cdot v_{p} \cdot e^{i \theta^{\prime}}}{\hbar_{x}}-\left(\frac{F . s . e^{i \theta^{\prime}}}{\hbar_{x}^{2}}\right) \cdot \frac{\partial \hbar_{x}}{\partial \tau} \\
& +i \frac{F . s . e^{i \theta^{\prime}}}{\hbar_{x}} \frac{\partial \theta^{\prime}}{\partial \tau}
\end{aligned}
$$

Now by putting equations (402) and (404) in equation (395), we get-

$$
\begin{aligned}
\frac{\partial \mathcal{P}(\tau, s)}{\partial \tau}=-\frac{i}{\hbar_{x}} & \mathcal{P}(\tau, s)\left[E-\left(\frac{E \cdot \tau}{\hbar_{x}}\right) \frac{\partial \hbar_{x}}{\partial \tau}+\tau \frac{\partial E}{\partial \tau}+i E \tau \cdot \frac{\partial \theta^{\prime}}{\partial \tau}\right. \\
& +\left(\frac{F \cdot s}{\hbar_{x}}\right) \cdot \frac{\partial \hbar_{x}}{\partial \tau}-s \cdot \frac{\partial F}{\partial \tau}-F \cdot v_{p} \\
& \left.-i F s \cdot \frac{\partial \theta^{\prime}}{\partial \tau}\right] e^{i \theta^{\prime}}
\end{aligned}
$$

Or

$$
\begin{aligned}
i \hbar_{x} \frac{\partial \mathcal{P}(\tau, s)}{\partial \tau}=\mathcal{P}(\tau, s)\left[\left(E-F . v_{p}\right)+\left(\tau \frac{\partial E}{\partial \tau}-s . \frac{\partial F}{\partial \tau}\right)\right. \\
+i \frac{\partial \theta^{\prime}}{\partial \tau}(E . \tau-F . s) \\
\left.+\frac{1}{\hbar_{x}} \frac{\partial \hbar_{x}}{\partial \tau}(F . s-E . \tau)\right] e^{i \theta^{\prime}}
\end{aligned}
$$

So, above equation is Generalized Schrödinger's equation in terms of Universal Mechanics. If we consider $F, s \& \hbar_{x}$ time independent or don't consider duality, $\phi-\psi$ transformation, 
Universal Relativity and transformation of spin, then equation (406) becomes-

$$
i \hbar_{x} \frac{\partial \mathcal{P}(\tau, s)}{\partial \tau}=E \cdot \mathcal{P}(\tau, s)
$$

This equation is time dependent Schrödinger's equation in quantum mechanical limit. So, as Generalized Correspondence Principle predicts Generalized Schrödinger's equation becomes Schrödinger's equation in case of quantum limit from universal case. Now if we differentiate wave function with respect to space parameter $s$, then-

$$
\begin{aligned}
\frac{\partial \mathcal{P}(\tau, s)}{\partial s}=A \frac{\partial}{\partial s}\left[e^{\frac{i}{\hbar_{x}}(F . s-E . \tau)}\right] \\
=i A e^{\frac{i}{\hbar_{x}}(F . s-E . \tau)}\left[\frac{\partial}{\partial s}\left(\frac{F . s}{\hbar_{x}}\right)-\frac{\partial}{\partial s}\left(\frac{E . \tau}{\hbar_{x}}\right)\right] \\
=i \mathcal{P}(\tau, s)\left[\frac{\partial}{\partial s}\left(\frac{F . s}{\hbar_{x}}\right)-\frac{\partial}{\partial s}\left(\frac{E . \tau}{\hbar_{x}}\right)\right]
\end{aligned}
$$

Now by differentiating again with respect to space parameter $s$, we get-

$$
\begin{aligned}
\frac{\partial^{2} \mathcal{P}(\tau, s)}{\partial s^{2}}=-A . e^{\frac{i}{\hbar_{x}}(F . s-E . \tau)}\left[\frac{\partial}{\partial s}\left(\frac{F . s}{\hbar_{x}}\right)-\frac{\partial}{\partial s}\left(\frac{E . \tau}{\hbar_{x}}\right)\right]^{2} \\
+i \mathcal{P}(\tau, s)\left[\frac{\partial^{2}}{\partial s^{2}}\left(\frac{F . s}{\hbar_{x}}\right)-\frac{\partial^{2}}{\partial s^{2}}\left(\frac{E . \tau}{\hbar_{x}}\right)\right]
\end{aligned}
$$

If $E, \tau, F \& \hbar_{x}$ are independent of space parameter $s$, then-

$$
\begin{gathered}
\frac{\partial^{2} \mathcal{P}(\tau, s)}{\partial s^{2}}=-\mathcal{P}(\tau, s)\left(\frac{F}{\hbar_{x}}\right)^{2} \\
\hbar_{x}^{2} \frac{\partial^{2} \mathcal{P}(\tau, s)}{\partial s^{2}}+F^{2} \cdot \mathcal{P}(\tau, s)=0 \\
\because F=\psi \cdot v_{p} \text { then } F^{2}=\psi^{2} \cdot v_{p}^{2}
\end{gathered}
$$

Now by putting the value of $F^{2}$ and dividing whole equation with $2 \psi$, we get-

$$
\begin{gathered}
\frac{\hbar_{x}^{2}}{2 \psi} \frac{\partial^{2} \mathcal{P}(\tau, s)}{\partial s^{2}}+\frac{1}{2} \psi v_{p}^{2} \cdot \mathcal{P}(\tau, s)=0 \\
\left\{\because E_{k}=\frac{1}{2} \psi v_{p}^{2}\right\}
\end{gathered}
$$

Then, above equation becomes-

$$
\frac{\hbar_{x}^{2}}{2 \psi} \frac{\partial^{2} \mathcal{P}(\tau, s)}{\partial s^{2}}+E_{k} \cdot \mathcal{P}(\tau, s)=0
$$

This is time independent Schrödinger equation.

Now $E \equiv E_{k}+V$ here is potential energy, then in classical limit-

$$
\frac{\hbar_{x}^{2}}{2 \psi} \frac{\partial^{2} \mathcal{P}(\tau, s)}{\partial s^{2}}+(E-V) \cdot \mathcal{P}(\tau, s)=0
$$

$$
\left\{\text { Or } E=E_{p}+E_{c} \equiv E_{k}+V \text { in Normal Case }\right\}
$$

Now by putting the value of $E \cdot \mathcal{P}(\tau, s)$ from equation (407) in quantum mechanical limit, we get-

$$
\frac{\hbar_{x}^{2}}{2 \psi} \frac{\partial^{2} \mathcal{P}(\tau, s)}{\partial s^{2}}-V \cdot \mathcal{P}(\tau, s)+i \hbar_{x} \frac{\partial \mathcal{P}(\tau, s)}{\partial \tau}=0
$$

But if we conclude $\phi-\psi$ transformation, transformation of spin, Universal Relativity and duality of $\phi, \psi \& \tau$ in equation (409), we get-

$$
\begin{aligned}
\frac{\partial}{\partial s}\left(\frac{F . s}{\hbar_{x}}\right)=\frac{\partial}{\partial s}( & \left.\frac{F . s \cdot e^{i \theta}}{\hbar_{x}}\right) \\
& =\frac{s \cdot e^{i \theta}}{\hbar_{x}} \frac{\partial F}{\partial s}+\frac{F \cdot e^{i \theta}}{\hbar_{x}}-\left(\frac{F . s \cdot e^{i \theta}}{\hbar_{x}^{2}}\right) \cdot \frac{\partial \hbar_{x}}{\partial s} \\
& +i \frac{F . s \cdot e^{i \theta}}{\hbar_{x}} \frac{\partial \theta}{\partial s}
\end{aligned}
$$

$$
\begin{gathered}
\left\{\because \theta=\theta_{\psi}+\theta_{\phi}-\theta_{\tau}\right\} \\
F=\psi \frac{\partial s}{\partial \tau} \operatorname{than} \frac{\partial F}{\partial s}=\frac{\partial \psi}{\partial s} \frac{\partial s}{\partial \tau}+\psi \frac{\partial}{\partial s}\left(\frac{\partial s}{\partial \tau}\right) \Rightarrow \frac{\partial \psi}{\partial \tau}+\psi \frac{\partial}{\partial s}\left(\frac{\partial s}{\partial \tau}\right)
\end{gathered}
$$

Now by putting value of $\frac{\partial F}{\partial s}$ from equation (417), we get equation (416) as-

$$
\begin{aligned}
\frac{\partial}{\partial s}\left(\frac{F . s . e^{i \theta}}{\hbar_{x}}\right)= & \frac{e^{i \theta}}{\hbar_{x}}\left[s \frac{\partial \psi}{\partial \tau}+\psi \cdot s \frac{\partial}{\partial s}\left(\frac{\partial s}{\partial \tau}\right)+F-\left(\frac{F \cdot s}{\hbar_{x}}\right) \cdot \frac{\partial \hbar_{x}}{\partial s}\right. \\
& \left.+i F s \cdot \frac{\partial \theta}{\partial s}\right]
\end{aligned}
$$

In similar way- 


$$
\begin{aligned}
\frac{\partial}{\partial s}\left(\frac{E . \tau}{\hbar_{x}}\right)=\frac{\partial}{\partial \tau}\left(\frac{E . \tau e^{i \theta^{\prime \prime}}}{\hbar_{x}}\right) & \\
& =\frac{e^{i \theta^{\prime \prime}}}{\hbar_{x}}\left[\tau \frac{\partial E}{\partial s}+E \frac{\partial \tau}{\partial s}-\left(\frac{E \cdot \tau}{\hbar_{x}}\right) \cdot \frac{\partial \hbar_{x}}{\partial \tau}\right. \\
& \left.+i E \cdot \tau \frac{\partial \theta^{\prime \prime}}{\partial \tau}\right]
\end{aligned}
$$

Now by putting $E=\phi \psi$, we get-

$$
\begin{gathered}
\frac{\partial E}{\partial s}=\phi \frac{\partial \psi}{\partial s}+\psi \frac{\partial \phi}{\partial s} \\
\frac{\partial}{\partial \tau}\left(\frac{E \cdot \tau e^{i \theta^{\prime \prime}}}{\hbar_{x}}\right)=\frac{e^{i \theta^{\prime \prime}}}{\hbar_{x}}\left[\tau \cdot \phi \frac{\partial \psi}{\partial s}+\tau \cdot \psi \frac{\partial \phi}{\partial s}+E \frac{1}{\partial s / \partial \tau}\right. \\
\left.-\left(\frac{E \cdot \tau}{\hbar_{x}}\right) \cdot \frac{\partial \hbar_{x}}{\partial \tau}+i E \cdot \tau \frac{\partial \theta^{\prime \prime}}{\partial \tau}\right]
\end{gathered}
$$

Now by putting $E$ outside to the bracket, we get-

$$
\begin{aligned}
\frac{\partial}{\partial \tau}\left(\frac{E \cdot \tau e^{i \theta^{\prime \prime}}}{\hbar_{x}}\right)= & \frac{E \cdot \tau \cdot e^{i \theta^{\prime \prime}}}{\hbar_{x}}\left[\frac{1}{\psi} \frac{\partial \psi}{\partial s}+\frac{1}{\phi} \frac{\partial \phi}{\partial s}+\frac{1}{\tau \cdot v_{p}}\right. \\
& \left.-\left(\frac{E \cdot \tau}{\hbar_{x}}\right) \cdot \frac{\partial \hbar_{x}}{\partial \tau}+i E \cdot \tau \frac{\partial \theta^{\prime \prime}}{\partial \tau}\right]
\end{aligned}
$$

In similar way equation (418) can be written as-

$$
\begin{aligned}
\frac{\partial}{\partial s}\left(\frac{F . s . e^{i \theta}}{\hbar_{x}}\right)= & \frac{F . s . e^{i \theta}}{\hbar_{x}}\left[\frac{1}{F} \frac{\partial \psi}{\partial \tau}+\frac{1}{v_{p}} \frac{\partial v_{p}}{\partial s}+\frac{1}{s}-\frac{1}{\hbar_{x}} \frac{\partial \hbar_{x}}{\partial s}\right. \\
& \left.+i \frac{\partial \theta}{\partial s}\right]
\end{aligned}
$$

Now by putting the values of first and second order derivatives of these functions in equation (409) from equations (422) and (433) or from appendix [A] equation (A3) \& (A-4), we get-

$$
\begin{aligned}
& \frac{\partial^{2} \mathcal{P}(\tau, s)}{\partial s^{2}}=-\mathcal{P}(\tau, s)\left[( \frac { F . s . e ^ { i \theta } } { \hbar _ { x } } ) \left\{\frac{1}{F} \frac{\partial \psi}{\partial \tau}+\frac{1}{v_{p}} \frac{\partial v_{p}}{\partial s}+\frac{1}{s}\right.\right. \\
& \left.-\frac{1}{\hbar_{x}} \frac{\partial \hbar_{x}}{\partial s}+i \frac{\partial \theta}{\partial s}\right\} \\
& -\frac{E \cdot \tau \cdot e^{i \theta^{\prime \prime}}}{\hbar_{x}}\left\{\frac{1}{\psi} \frac{\partial \psi}{\partial s}+\frac{1}{\phi} \frac{\partial \phi}{\partial s}+\frac{1}{\tau \cdot v_{p}}\right. \\
& \left.\left.-\left(\frac{E \cdot \tau}{\hbar_{x}}\right) \cdot \frac{\partial \hbar_{x}}{\partial \tau}+i E \cdot \tau \frac{\partial \theta^{\prime \prime}}{\partial \tau}\right\}\right]^{2} \\
& +i \mathcal{P}(\tau, s)\left[( \frac { F . s . e ^ { i \theta } } { \hbar _ { x } } ) \left\{\left(\frac{1}{F} \frac{\partial \psi}{\partial \tau}+\frac{1}{v_{p}} \frac{\partial v_{p}}{\partial s}\right.\right.\right. \\
& \left.+\frac{1}{s}-\frac{1}{\hbar_{x}} \frac{\partial \hbar_{x}}{\partial s}+i \frac{\partial \theta}{\partial s}\right)^{2} \\
& +\left(-\frac{1}{F^{2}} \frac{\partial F}{\partial s} \frac{\partial \psi}{\partial \tau}+\frac{1}{F} \frac{\partial}{\partial s}\left(\frac{\partial \psi}{\partial \tau}\right)-\frac{1}{v_{p}^{2}}\left(\frac{\partial v_{p}}{\partial s}\right)^{2}\right. \\
& +\frac{1}{v_{p}} \frac{\partial^{2} v_{p}}{\partial s^{2}}-\frac{1}{s^{2}}+\frac{1}{\hbar_{x}^{2}}\left(\frac{\partial \hbar_{x}}{\partial s}\right)^{2}-\frac{1}{\hbar_{x}} \frac{\partial^{2} \hbar_{x}}{\partial s^{2}} \\
& \left.\left.+i \frac{\partial^{2} \theta}{\partial s^{2}}\right)\right\} \\
& -\left(\frac{E \cdot \tau \cdot e^{i \theta^{\prime \prime}}}{\hbar_{x}}\right)\left\{\left(\frac{1}{\psi} \frac{\partial \psi}{\partial s}+\frac{1}{\phi} \frac{\partial \phi}{\partial s}+\frac{1}{\tau \cdot v_{p}}\right.\right. \\
& \left.-\left(\frac{E \cdot \tau}{\hbar_{x}}\right) \cdot \frac{\partial \hbar_{x}}{\partial \tau}+i E \cdot \tau \frac{\partial \theta^{\prime \prime}}{\partial \tau}\right) \\
& +\left(\frac{1}{\phi} \frac{\partial^{2} \phi}{\partial s^{2}}+\frac{1}{\psi} \frac{\partial^{2} \psi}{\partial s^{2}}-\frac{1}{\phi^{2}}\left(\frac{\partial \phi}{\partial s}\right)^{2}\right. \\
& -\frac{1}{\psi^{2}}\left(\frac{\partial \psi}{\partial s}\right)^{2}-\frac{1}{\tau v_{p}}\left(\frac{1}{\tau v_{p}}+\frac{1}{v_{p}} \frac{\partial v_{p}}{\partial s}\right) \\
& \left.\left.\left.+\frac{1}{\hbar_{x}^{2}}\left(\frac{\partial \hbar_{x}}{\partial s}\right)^{2}-\frac{1}{\hbar_{x}} \frac{\partial^{2} \hbar_{x}}{\partial s^{2}}+i \frac{\partial^{2} \theta^{\prime \prime}}{\partial s^{2}}\right)\right\}\right]
\end{aligned}
$$

Now by solving above equation and from appendix [A] equation $(\mathrm{A}-9)$, we get-

$$
i \frac{\partial^{2} \mathcal{P}(\tau, s)}{\partial s^{2}}+\mathcal{P} . \mathcal{P}(\tau, s)=0
$$

So, this is the general form of time independent Generalized Schrödinger's equation.

Here parameter $P$ is-

$$
\begin{gathered}
\mathbb{P}=\left\{k_{1} \mathfrak{B}^{2}\left(1+i k_{1}\right)+k_{2} \mathfrak{D}^{2}\left(i k_{2}-1\right)-2 i k_{1} k_{2} \mathfrak{B D}+k_{1} \mathfrak{B}^{\prime}\right. \\
\left.-k_{2} \mathfrak{D}^{\prime}\right\}
\end{gathered}
$$

Usually this parameter changes in different situations. So like in certain condition $\hbar_{x}$ and $\theta^{\prime \prime}$ are linearly depends upon space 
parameter, then this equation will have different values. Here from appendix [A], we know the parameters-

$$
\begin{gathered}
k_{1}=\left(\frac{F \cdot s \cdot e^{i \theta}}{\hbar_{x}}\right) \\
k_{2}=\left(\frac{E \cdot \tau \cdot e^{i \theta^{\prime \prime}}}{\hbar_{x}}\right)
\end{gathered}
$$

$$
\begin{aligned}
\mathfrak{B}=\frac{1}{k_{1}} \frac{\partial k_{1}}{\partial s}=\frac{k_{1}^{\prime}}{k_{1}}=\left\{\frac{1}{F} \frac{\partial \psi}{\partial \tau}+\frac{1}{v_{p}} \frac{\partial v_{p}}{\partial s}+\frac{1}{s}-\frac{1}{\hbar_{x}} \frac{\partial \hbar_{x}}{\partial s}+i \frac{\partial \theta}{\partial s}\right\} \\
\mathfrak{D}=\frac{1}{k_{2}} \frac{\partial k_{2}}{\partial s}=\frac{k_{2}^{\prime}}{k_{2}}=\left\{\frac{1}{\psi} \frac{\partial \psi}{\partial s}+\frac{1}{\phi} \frac{\partial \phi}{\partial s}+\frac{1}{\tau \cdot v_{p}}-\left(\frac{E \cdot \tau}{\hbar_{x}}\right) \cdot \frac{\partial \hbar_{x}}{\partial \tau}\right. \\
\left.+i E \cdot \tau \frac{\partial \theta^{\prime \prime}}{\partial \tau}\right\}
\end{aligned}
$$

Now by putting the values of and from equations (429) and (430), we get equation (426) as-

$$
\begin{aligned}
\mathbb{P}=\left\{k_{1} \frac{\left(k_{1}^{\prime}\right)^{2}}{k_{1}^{2}}(1\right. & \left.+i k_{1}\right)+k_{2} \frac{\left(k_{2}^{\prime}\right)^{2}}{k_{2}^{2}}\left(i k_{2}-1\right)-2 i k_{1}^{\prime} k_{2}^{\prime} \\
& \left.+k_{1} \mathfrak{B}^{\prime}-k_{2} \mathfrak{D}^{\prime}\right\} \\
& =\frac{\left(k_{1}^{\prime}\right)^{2}}{k_{1}}\left(1+i k_{1}\right)+\frac{\left(k_{2}^{\prime}\right)^{2}}{k_{2}}\left(i k_{2}-1\right) \\
& -2 i k_{1}^{\prime} k_{2}^{\prime}-\frac{k_{1}^{\prime}}{k_{1}}+\frac{\left(k_{2}^{\prime}\right)^{2}}{k_{2}}+k_{1}^{\prime \prime}-k_{2}^{\prime \prime}
\end{aligned}
$$

$$
\left\{\because \mathfrak{B}^{\prime}=\frac{k_{1}^{\prime \prime}}{k_{1}}-\frac{k_{1}^{\prime}}{k_{1}^{2}}, \mathfrak{D}^{\prime}=\frac{k_{2}^{\prime \prime}}{k_{2}}-\frac{k_{2}^{\prime}}{k_{2}^{2}}\right\}
$$

Now by simplification, we get above equation as-

$$
\begin{gathered}
\mathrm{P}=\left\{k_{1}^{\prime \prime}-k_{2}^{\prime \prime}+\frac{\left(k_{1}^{\prime}\right)^{2}}{k_{1}}-\frac{\left(k_{1}^{\prime}\right)^{2}}{k_{1}}-\frac{\left(k_{2}^{\prime}\right)^{2}}{k_{2}}+i\left(k_{1}^{\prime}-k_{2}^{\prime}\right)^{2}\right\} \\
=\left\{\left(k_{1}^{\prime \prime}-k_{2}^{\prime \prime}\right)+i\left(k_{1}^{\prime}-k_{2}^{\prime}\right)^{2}\right\}
\end{gathered}
$$

Now by putting the value of in equation (425), we get-

$$
i \frac{\partial^{2} \mathcal{P}(\tau, s)}{\partial s^{2}}+\left\{\left(k_{1}^{\prime \prime}-k_{2}^{\prime \prime}\right)+i\left(k_{1}^{\prime}-k_{2}^{\prime}\right)^{2}\right\} . \mathcal{P}(\tau, s)=0
$$

Now as we know, we put here-

$$
\mathcal{P}(\tau, s)=A e^{\frac{i}{\mathrm{~h}_{x}}(F . s-E . \tau)}=A e^{i\left\{\left(\frac{F . S}{\mathrm{\hbar}_{x}}\right)-\left(\frac{E . \tau}{\mathrm{h}_{x}}\right)\right\}}=A e^{i\left(k_{1}-k_{2}\right)}
$$

We can also get above equation (433) by differentiating two times to above equation, as-

$$
\frac{\partial \mathcal{P}(\tau, s)}{\partial s}-A \cdot i\left(k_{1}^{\prime}-k_{2}^{\prime}\right) \cdot e^{i\left(k_{1}-k_{2}\right)}=i\left(k_{1}^{\prime}-k_{2}^{\prime}\right) \cdot \mathcal{P}(\tau, s)
$$

Or

$$
\begin{array}{r}
\frac{\partial^{2} \mathcal{P}(\tau, s)}{\partial s^{2}}=A i^{2}\left(k_{1}^{\prime}-k_{2}^{\prime}\right)^{2} \cdot e^{i\left(k_{1}-k_{2}\right)}+i \cdot \mathcal{P}(\tau, s)\left(k_{1}^{\prime \prime}-k_{2}^{\prime \prime}\right) \\
=-\left(k_{1}^{\prime}-k_{2}^{\prime}\right)^{2} \mathcal{P}(\tau, s)+i \mathcal{P}(\tau, s)\left(k_{1}^{\prime \prime}-k_{2}^{\prime \prime}\right)
\end{array}
$$

Now by multiplying both sides with $i$, we get-

$$
i \frac{\partial^{2} \mathcal{P}(\tau, s)}{\partial s^{2}}=-i\left(k_{1}^{\prime}-k_{2}^{\prime}\right)^{2} \mathcal{P}(\tau, s)-\mathcal{P}(\tau, s)\left(k_{1}^{\prime \prime}-k_{2}^{\prime \prime}\right)
$$

Or

$$
i \frac{\partial^{2} \mathcal{P}(\tau, s)}{\partial s^{2}}=-\mathcal{P}(\tau, s)\left\{\left(k_{1}^{\prime \prime}-k_{2}^{\prime \prime}\right)+i\left(k_{1}^{\prime}-k_{2}^{\prime}\right)^{2}\right\}
$$

Or

$$
i \frac{\partial^{2} \mathcal{P}(\tau, s)}{\partial s^{2}}+\mathcal{P}(\tau, s)\left\{\left(k_{1}^{\prime \prime}-k_{2}^{\prime \prime}\right)+i\left(k_{1}^{\prime}-k_{2}^{\prime}\right)^{2}\right\}=0
$$

Or

$$
i \frac{\partial^{2} \mathcal{P}(\tau, s)}{\partial s^{2}}+\mathcal{P} \cdot \mathcal{P}(\tau, s)=0
$$

So, this equation is valid in all type of Central Systems with including $\phi-\psi$ transformation, transformation of spin, variation in the speed of broken parts etc. This can also predict about Solar systems and galaxies also.

Now if the curvature in space time caused by $\mathrm{n}^{\text {th }}$ variation in scalar field density, than field equation for General Relativity becomes in my theoretical sense as-

$$
\sum_{i=1}^{n} \Delta^{i} \rho_{\phi}=G_{x} E
$$


This equation is valid for minor singularities (black holes etc) also.

As we know $\Delta \rho_{\phi}$ or $\delta \rho_{\phi}$ produces normally Central System force but in different cases it produces differently $\delta^{2} \rho_{\phi}, \delta^{3} \rho_{\phi} \ldots \delta^{n} \rho_{\phi}$ (like in case of minor singularities). In more detailed version this will be discussed by me in my next article on unification of all fundamental forces.

\subsection{Generalized Klein-Gordon And Dirac Equations}

Now I intend to define Klein-Jordan [19] and Paul Dirac's equations [20] in Generalized or universal way. To get Klein-Jordan equation in form of $E=\phi \psi$, we have to use dispersion relation in different and unique or universal form.

$$
\begin{gathered}
E^{2}=E_{p}^{2}+E_{c}^{2}+2 E_{p} E_{c} \\
\left\{E=\phi \psi, E_{p}=\psi_{p} \phi, E_{c}=\alpha \phi \Delta \phi\right\} \\
\psi^{2} \phi^{2}=\psi_{p}^{2} \phi^{2}+\alpha^{2}(\Delta \phi)^{2} \phi^{2}+2 \phi^{2} \psi_{p}(\alpha \Delta \phi) \\
E_{c} \equiv E_{k}=\frac{1}{2} \psi v_{p}^{2}=\frac{\left(\psi v_{p}\right)^{2}}{2 \psi}=\frac{F^{2}}{2 \psi}=T
\end{gathered}
$$

Now by Einstein's dispersion relation [7]-

$$
E^{2}=p^{2} c^{2}+m_{0}^{2} c^{4}
$$

Usually Klein-Jordan equation is defined as-

$$
\left(\partial^{\mu} \partial_{\mu}-m^{2} c^{2}\right) \mathcal{P}=0
$$

Four momentums of bodies-

$$
\begin{aligned}
p_{\mu} p^{\mu}=p_{0} p^{0}+ & p_{1} p^{1}+p_{2} p^{2}+p_{3} p^{3} \\
& =\left(i \hbar \frac{\partial}{\partial t}\right)^{2}+\left(-i \hbar \frac{\partial}{\partial x}\right)^{2}+\left(-i \hbar \frac{\partial}{\partial y}\right)^{2} \\
& +\left(-i \hbar \frac{\partial}{\partial z}\right)^{2}=-\hbar^{2}\left(\frac{\partial^{2}}{\partial t^{2}}+\nabla^{2}\right)
\end{aligned}
$$

Here $\hat{E}=i \hbar \frac{\partial}{\partial t}$ and $\hat{p}=-i \hbar \nabla$ are respectively energy and momentum (Quantity of Motion in Universal Sense) operators.

But in form of my predefined wave function (valid in all Central Systems), we get-

$$
\mathcal{P}(\tau, s)=A e^{\frac{i}{\hbar_{x}}(F . s-E . \tau)}
$$

From Generalized Schrödinger's equation we already know that $i \hbar \frac{\partial}{\partial \tau}$ can't be energy operator in this case. So, what will be the simplified forms of energy and momentum four vectors and operators? Formerly as we know D'Alembert's Operator-

$$
\square=\left(\frac{1}{c^{2}} \frac{\partial^{2}}{\partial t^{2}}-\Delta\right)
$$

But, in this case $k_{1}$ and $k_{2}$ have taken place on the place of $\hat{E}$ and $\hat{p}$ because we have four variables in our wave function in universal sense. Now from equation (444), we can conclude that-

$$
\frac{1}{2} \psi v_{p}^{2} \equiv \alpha \phi \Delta \phi\left\{\because \alpha \phi=\psi^{\prime}\right\}
$$

This case only holds if converts the energy only created due to motion in body.

$$
v_{p}^{2}=2\left(\frac{\psi^{\prime}}{\psi}\right) \Delta \phi=2 \beta \Delta \phi \quad\left\{\because \beta=\frac{\alpha \phi}{\psi}=\frac{\psi^{\prime}}{\psi}\right\}
$$

This case propagation velocity can be defined as-

$$
v_{p}= \pm \sqrt{2 \beta \Delta \phi}
$$

Or

$$
\Delta \phi=\frac{v_{p}^{2}}{2 \beta}
$$

So, in this case we can conclude value of variation in scalar field if we know values of velocity and $\beta$ for body. Now in simple case if $F, s, \hbar_{x}$ or $E$ are independent of time parameter, then-

$$
\begin{gathered}
\frac{\partial \mathcal{P}(\tau, s)}{\partial \tau}=-\frac{i}{\hbar_{x}} E \cdot \mathcal{P}(\tau, s) \\
i \hbar_{x} \frac{\partial \mathcal{P}(\tau, s)}{\partial \tau}=E \cdot \mathcal{P}(\tau, s)
\end{gathered}
$$

In this case we got energy operator as-

$$
\hat{E}=i \hbar \frac{\partial}{\partial \tau}
$$


Now by using $F=\psi v_{p}$ and relation (448), we get-

$$
E_{c}=\alpha \phi \Delta \phi=\alpha \phi \frac{v_{p}^{2}}{2 \beta} \equiv E_{k}=\frac{1}{2} \psi v_{p}^{2}=\frac{F^{2}}{2 \psi}
$$

Now for simplicity if $F, s, \hbar_{x} \& \tau$ are independent of space parameter $s$, then-

$$
\frac{\partial^{2} \mathcal{P}(\tau, s)}{\partial s^{2}}=-\frac{F^{2}}{\hbar_{x}^{2}} \mathcal{P}(\tau, s)
$$

Or

$$
-\frac{\hbar_{x}^{2}}{2 \psi} \frac{\partial^{2} \mathcal{P}(\tau, s)}{\partial s^{2}}=\frac{F^{2}}{2 \psi} \mathcal{P}(\tau, s)=E_{c} \mathcal{P}(\tau, s)
$$

In this case we get converged energy operator as-

$$
\widehat{E}_{c}=-\frac{\hbar_{x}^{2}}{2 \psi} \frac{\partial^{2}}{\partial s^{2}}
$$

Or we can write equation (453) as-

$$
\frac{\partial^{2} \mathcal{P}(\tau, s)}{\partial s^{2}}=-\frac{\psi^{2} v_{p}^{2}}{\hbar_{x}^{2}} \mathcal{P}(\tau, s)
$$

Now by putting $\psi=\psi_{p}+\alpha \Delta \phi$, we get-

$$
-\frac{\hbar_{x}^{2}}{v_{p}^{2}} \frac{\partial^{2} \mathcal{P}(\tau, s)}{\partial s^{2}}=\left(\psi_{p}^{2}+\alpha^{2}(\Delta \phi)^{2}+2 \psi_{p}(\alpha \Delta \phi)\right) \mathcal{P}(\tau, s)
$$

Now by multiplying both sides with $\phi^{2}$, we get-

$$
\begin{aligned}
-\frac{\hbar_{x}^{2} \phi^{2}}{v_{p}^{2}} \frac{\partial^{2} \mathcal{P}(\tau, s)}{\partial s^{2}} & \\
= & \left(\psi_{p}^{2} \phi^{2}+\alpha^{2}(\Delta \phi)^{2} \phi^{2}\right. \\
& \left.+2 \phi^{2} \psi_{p}(\alpha \Delta \phi)\right) \mathcal{P}(\tau, s)
\end{aligned}
$$

So, we can write this equation as-

$$
\left(E^{2}+\frac{\hbar_{x}^{2} \phi^{2}}{v_{p}^{2}} \nabla^{2}\right) \mathcal{P}(\tau, s)=0
$$

Now by putting a question (451) and energy operator in above equation, we get-

$$
\left(\left(i \hbar_{x} \frac{\partial}{\partial \tau}\right)^{2}+\frac{\hbar_{x}^{2} \phi^{2}}{v_{p}^{2}} \nabla^{2}\right) \mathcal{P}(\tau, s)=0
$$

Or

$$
\left(-\hbar_{x}^{2} \frac{\partial^{2}}{\partial \tau^{2}}+\frac{\hbar_{x}^{2} \phi^{2}}{v_{p}^{2}} \nabla^{2}\right) \mathcal{P}(\tau, s)=0
$$

Now by removing similar terms and by some manipulation, we get-

$$
\left(-v_{p}^{2} \frac{\partial^{2}}{\partial \tau^{2}}+\phi^{2} \nabla^{2}\right) \mathcal{P}(\tau, s)=0
$$

Now by putting-

$$
\mathrm{A}\left[=-v_{p}^{2} \frac{\partial^{2}}{\partial \tau^{2}}+\phi^{2} \frac{\partial^{2}}{\partial s^{2}}\right.
$$

We get above equation as-

$$
\text { 口पA.P } \mathcal{P}(\tau, s)=0
$$

This is a unique form of Dirac equation in universal sense because it holds for bodies which have $\psi_{p} \neq 0$ but it also holds for clean Klein-Jordan equation by putting for broken parts. This operator $\mathrm{A}$ also holds in n-dimensions according to body's dimensions.

Now as we know for broken parts $\psi_{p_{b}}=0$. So-

$$
E=E_{c}
$$

Or

$$
i \hbar_{x} \frac{\partial \mathcal{P}(\tau, s)}{\partial \tau}=-\frac{\hbar_{x}^{2}}{2 \psi_{b}} \frac{\partial^{2} \mathcal{P}(\tau, s)}{\partial s^{2}}
$$




$$
\left(i \hbar_{x} \frac{\partial}{\partial \tau}+\frac{\hbar_{x}^{2}}{2 \psi_{b}} \nabla^{2}\right) \mathcal{P}(\tau, s)=0
$$

(466)

Now by removing similar terms and by some manipulations, we get-

$$
\left(i 2 \psi_{b} \frac{\partial}{\partial \tau}+\hbar_{x} \nabla^{2}\right) \mathcal{P}(\tau, s)=0
$$

Or

$$
\mathrm{A}_{\mathrm{b}}=i 2 \psi_{b} \frac{\partial}{\partial \tau}+\hbar_{x} \nabla^{2}
$$

So, we get equation (467) as-

$$
\mathrm{A}_{\mathrm{b}} \cdot \mathcal{P}(\tau, s)=0
$$

This operator also can be written as-

$$
\frac{\psi_{b}}{2}\left(v_{p}\right)_{c}^{2}=\Delta \psi_{b} . \phi_{b}=\psi_{b} \phi_{b}=E_{b}
$$

Now by you using equation (106) and multiplying it with $\phi^{2}$ both sides, we get-

$$
E^{2} \phi^{2}=\psi^{2} \phi^{2} \epsilon^{2}+\psi_{p}^{2} \phi^{2} \epsilon_{c}^{2}
$$

Or

$$
E^{2}\left(\phi^{2}-\epsilon^{2}\right)=E_{p}^{2} \epsilon_{c}^{2}
$$

Or

$$
\begin{gathered}
\frac{E^{2}}{E_{p}^{2}}\left(\phi^{2}-\epsilon^{2}\right)=\epsilon_{c}^{2} \\
\left(1+\frac{\alpha \Delta \phi}{\psi_{p}}\right)^{2}\left(\phi^{2}-\epsilon^{2}\right)=\epsilon_{c}^{2} \\
\left\{\because \frac{\alpha \Delta \phi}{\psi_{p}}=\gamma\right\}
\end{gathered}
$$

Or

$$
(1+\gamma)^{2}\left(\phi^{2}-\epsilon^{2}\right)=\epsilon_{c}^{2}
$$

$$
\phi^{2}=\epsilon^{2}+\frac{\epsilon_{c}^{2}}{(1+\gamma)^{2}}
$$

So, this is a special relativistic case for my equations. Now if we put a special relativistic case in my equation (462), then-

$$
\left(-v_{p}^{2} \frac{\partial^{2}}{\partial \tau^{2}}+\left(\epsilon^{2}+\frac{\epsilon_{c}^{2}}{(1+\gamma)^{2}}\right) \nabla^{2}\right) \mathcal{P}(\tau, s)=0
$$

$$
\left\{\because \epsilon^{2}=v_{p}^{2}\left(v_{p}\right)_{c}^{2}, \epsilon_{c}^{2}=\left(v_{p}\right)_{c}^{4}\right\}
$$

$$
\left\{-\frac{\partial^{2}}{\partial \tau^{2}}+\left(v_{p}\right)_{c}^{2}\left(1+\frac{1}{(1+\gamma)^{2}}\left(\frac{\left(v_{p}\right)_{c}}{v_{p}}\right)^{2}\right) \nabla^{2}\right\} \mathcal{P}(\tau, s)=0
$$

$\because \frac{v_{p}}{\left(v_{p}\right)_{c}}=\beta_{r}\left(\right.$ Relativistic $\beta$ parameter $\left.\beta_{r}=\frac{v}{c}\right)$

Therefore-

$$
\left\{-\frac{1}{\left(v_{p}\right)_{c}^{2}} \frac{\partial^{2}}{\partial \tau^{2}}+\left(1+\frac{1}{\left(\beta_{r}(1+\gamma)\right)^{2}}\right) \nabla^{2}\right\} \mathcal{P}(\tau, s)=0
$$

And this shows a tendency towards D'Alembert's wave operator.

Now as we know in normal case-

$$
E=E_{p}+E_{c}
$$

$$
\begin{gathered}
\left(i \hbar_{x} \frac{\partial}{\partial \tau}+\frac{\hbar_{x}^{2}}{2 \psi} \nabla^{2}-E_{p}\right) \mathcal{P}(\tau, s)=0 \\
\left(\psi i \hbar_{x} \frac{\partial}{\partial \tau}+\frac{\hbar_{x}^{2}}{2} \nabla^{2}-\psi_{p}(\psi \phi)\right) \mathcal{P}(\tau, s)=0
\end{gathered}
$$




$$
\begin{gathered}
\left(\psi i \hbar_{x} \frac{\partial}{\partial \tau}+\frac{\hbar_{x}^{2}}{2} \nabla^{2}-\psi_{p} i \hbar_{x} \frac{\partial}{\partial \tau}\right) \mathcal{P}(\tau, s)=0 \\
\left(\left(\psi-\psi_{p}\right) i \hbar_{x} \frac{\partial}{\partial \tau}+\frac{\hbar_{x}^{2}}{2} \nabla^{2}\right) \mathcal{P}(\tau, s)=0
\end{gathered}
$$

Or

$$
\left(\alpha \Delta \phi . i \hbar_{x} \frac{\partial}{\partial \tau}+\frac{\hbar_{x}^{2}}{2} \nabla^{2}\right) \mathcal{P}(\tau, s)=0
$$

Or

$$
\left(i \hbar_{x} \frac{\partial}{\partial \tau}+\frac{\hbar_{x}^{2}}{2 \Delta \psi} \nabla^{2}\right) \mathcal{P}(\tau, s)=0
$$

Or

$$
\left(i \frac{2 \Delta \psi}{\hbar_{x}} \frac{\partial}{\partial \tau}+\nabla^{2}\right) \mathcal{P}(\tau, s)=0
$$

This equation is general form of Dirac and Klein-Jordan equations both or holds for all kind of quantum bodies (according to observer O' in Central Relativistic Case). In this equation we can clearly notice that if there is no $\phi-\psi$ transformation then, there is no Wave-Particle Duality in nature because $\Delta \psi=0$. So, the first part with time derivative vanishes and only remains to a normal Laplace equation which only shows a particular nature (whether wave or particle nature) not both. As I conjectured earlier in the paper that Wave-Particle Duality and Uncertainty in nature are due to $\phi-\psi$ transformation proven by this particular equation. Now what will happen in general case where is not independent of time and space parameters?

At first go through some general things which are so much important to this derivation like if we divide $E_{p}$ with $E_{c}$, then-

$$
\frac{E_{p}}{E_{c}}=\frac{\psi_{p}}{\alpha \Delta \phi}=\frac{1}{\gamma}
$$

Or

$$
\frac{E}{E_{c}}=\frac{\left(\psi_{p}+\alpha \Delta \phi\right)}{\alpha \Delta \phi}=\left(\frac{1}{\gamma}+1\right)
$$

Hence-

$$
\frac{E}{E_{c}}-\frac{E_{p}}{E_{c}}=1
$$

$$
\beta=\frac{\psi}{\alpha \phi}=\frac{\psi \phi}{\alpha \phi \cdot \phi}=\frac{E(\alpha \Delta \phi)}{\gamma(\alpha \phi) \cdot \psi_{p} \phi}=\frac{E}{\gamma E_{p}}\left(\frac{\Delta \phi}{\phi}\right)
$$

Therefore-

$$
\left(\frac{\Delta \phi}{\phi}\right)=\frac{\gamma \beta E_{p}}{E} \Rightarrow \frac{E_{c}}{\alpha \phi^{2}}=\frac{\gamma \beta E_{p}}{E}
$$

Hence-

$$
\phi^{2}=\frac{E E_{c}}{\alpha \beta \gamma E_{p}}
$$

Now from my former paper [6], we know that Heisenberg's Uncertainty relation proves that during inflations energy of Universe (dynamical caused by transformation in Universe) is positive but what if we get equation (443) in my former article as-

$$
\frac{1}{\Phi_{\text {atomic }} \Gamma\left(\Phi_{\text {at. }}\right)}\left\{\psi_{\text {atomic }} \Delta \Phi_{\text {atomic }}-\Phi_{\text {atomic }} \Delta \psi_{\text {atomic }}\right\} \geq \frac{\hbar}{2}
$$

In this case dynamical energy goes negative hope Universe and it starts deflating. So, Universe also has -ve Dynamical Energy during deflations.

$$
\psi_{\text {atomic }} \leq \alpha \Phi_{\text {atomic }}
$$

So, during deflations-

$$
\psi \leq \alpha \phi
$$

Or we can write it as-

$$
\frac{\psi}{\alpha \phi} \leq 1 \Rightarrow 1 \geq \frac{\alpha \phi}{\psi}
$$




$$
\beta_{d} \geq 1
$$

From my former paper [6], we know that-

$$
\beta_{i} \leq 1
$$

Here $d$ refers deflation and $i$ refers inflation.

\subsection{Generalized Complementary Principle and Max Born's Probability Density}

Now I intend to generalize a very renowned Principle in Quantum Mechanics known as "Complementary Principle" [33] in universal sense. So, the former statement of Complementary Principle is- "Complete knowledge of the particle aspects of a system (example path) is not compatible with complete knowledge of its wave aspects (example interference pattern)". Or in simple words, for a quantum system its particle aspects are complementary to its wave aspects.

Now in general case if an observer $\mathrm{O}^{\prime}$ is looking at just shorter bodies (if observer $\mathrm{O}^{\prime}$ is in $\mathrm{n}^{\text {th }}$ bodies, then he should look on ( $\mathrm{n}-1)^{\text {th }}$ bodies), then by Principle of Central System Relativity he will observe physical laws of Generalized Quantum Mechanics (or Quantum Mechanics in universal sense as obtained in this whole paper in form of Generalized Uncertainty Principle, Generalized Correspondence Principle, Generalized Schrödinger's equation common Generalized Wave-Particle Duality or Dirac and Klein-Klein-Jordan equation etc.). So, the statement of Complementary Principle is closely related to $\phi-\psi$ transformation in system or particles (shorter bodies) because at a particular instant of a particle (according to observer O' shorter bodies) is transformed into scalar field by $\phi-\psi$ transformation, then it will show wave aspect but when the particle transformed again quantity then it will show particle aspects. So, these both aspects can't happen for observer $\mathrm{O}$ ' at same instant or these are complementary to each other. So, the generalized statement of Complementary Principle becomes- "According to observer O' in Central Relativistic Case (In which the observer observes Generalized Quantum Mechanical laws) the wave and particle aspects of a system are complementary to each other and observed due to $\phi-\psi$ transformation in system". Or in other words "For observer O' complete knowledge of wave aspects (generated due to $\phi-\psi$ transformation in scalar field from quantity) is not compatible with the particle aspects (generated due to $\phi-\psi$ transformation of scalar field to quantity-more visible case) of that particular system because these both occurs at different instances or never occurs at same instant because same quantity transforms in scalar field and vice versa".

Now as we know Max Born's probability amplitude [15]-

$$
P(\tau, s)=|\mathcal{P}(\tau, s)|^{2}
$$

Now probability of finding a body (shorter according to observer O') in infinitesimal space $d s$ can be written as-

$$
d P(\tau, s)=|\mathcal{P}(\tau, s)|^{2} d s
$$

Or probability density can be written as-

$$
\frac{d P(\tau, s)}{d s}=|\mathcal{P}(\tau, s)|^{2}=\rho_{P}(\tau, s)
$$

So, probability of finding a body in Range $\left[s_{1}, s_{2}\right]$ is-

$$
\left[s_{1} ; s_{2}\right]=\int_{s_{1}}^{s_{2}} \rho_{P}(\tau, s) \cdot d s
$$

Or, from here Normalization Condition comes out as-

$$
\int_{-\infty}^{\infty} \rho_{P}(\tau, s) \cdot d s=1
$$

So, Normalization Condition states that neither a particle neither created nor destroyed in Universe but we know that if anything exists in Universe, then somehow it was created or in some way it will be destroyed. So, is there any Generalized version of Normalization Condition or not?

At first, let's go through Generalized Klein-Jordan equation from appendix equation [B-14] as-

$$
\left[2 \Delta \psi \cdot \nabla_{\tau}^{\prime}-\left(\nabla_{s}^{\prime}\right)^{2}\right] \mathcal{P}(\tau, s)=0
$$

This equation is valid in all kind of situations like transformation of spin, Central System Relativity, Universal Relativity, duality in time, space or quantity and scalar field etc. Here-

$$
\nabla_{\tau}^{\prime}=\left(i \hbar_{x} \frac{\partial}{\partial \tau}+\hat{\mathcal{Q}}_{\tau}\right) \& \nabla_{s}^{\prime}=-\left(i \hbar_{x} \frac{\partial}{\partial s}+\hat{\mathcal{Q}}_{s}\right)
$$

These are extended operators.

Now special case equation (486) can also be written as-

$$
\left[2 \Delta \psi \cdot \nabla_{\tau}-\nabla_{s}^{2}\right] \mathcal{P}(\tau, s)=0
$$


Over here-

$$
\nabla_{\tau}=i \hbar_{x} \frac{\partial}{\partial \tau} \& \nabla_{s}=\left(-i \hbar_{x} \frac{\partial}{\partial s}\right)
$$

So, in this form we can write above equation operators in form of extended operators as-

$$
\nabla_{\tau}^{\prime}=\nabla_{\tau}+\hat{\mathcal{Q}}_{\tau}
$$

Or

$$
\nabla_{s}^{\prime}=\nabla_{s}-\hat{\mathcal{Q}}_{s}
$$

Now one query must hitting your mental lexicon that what kind of solutions are generated by this extended and Generalized Glenn Klein-Jordan Dirac equation and how it is valid for both kind of particles fermions and Bosons, This equation is similar as heat equation [34]?

Now I intend to define equation (504) for broken parts and as we know for broken parts, we can write. So, equation (504) becomes-

$$
\left[2 \psi_{b} . \nabla_{\tau}^{\prime}-\left(\nabla_{s}^{\prime}\right)^{2}\right] \mathcal{P}(\tau, s)=0
$$

This particular equation is valid for all kind of broken parts (like in atomic sense- photos, in solar system sense- asteroids) according to observer $\mathrm{O}^{\prime}$ in Central Relativistic Case. This equation is similar as equation (467) previously defined for broken parts. In this case $\nabla_{s}^{\prime}$ including $i \hbar_{x}$ in itself and this produces negative sign.

\subsection{Generalized Matrix Mechanics}

Now I intend to define Matrix Mechanics in terms of Generalized Quantum Mechanics and how the operators evolves in this case like formerly evolution of quantum operators was defined by Werner Heisenberg evolution equation [36]. Let's go through the derivation of Heisenberg's derivation, as Langrangian $L(q, \dot{q})$ of action can be written as-

$$
\dot{q}=\frac{d q}{d t}
$$

And action can be written as-

$$
\mathcal{A}=\int L(q, \dot{q}) \cdot d t
$$

Now by variation in action equal to zero, we can write it as-

$$
\delta \mathcal{A}=\int \delta L(q, \dot{q}) \cdot d t=0
$$

Or

$$
-\frac{d}{d t} \cdot \frac{\partial L}{\partial \dot{q}}+\frac{\partial L}{\partial q}=0
$$

This is the famous Euler-Langrange equation [37]. We can also define Hamiltonian [38] of a Langrangian as-

$$
H=p \dot{q}-L
$$

So, this is a function of the coordinate $q$ and its conjugate momentum $p$ (quantity of motion $F$ ) as-

$$
p=\frac{\partial L}{\partial \dot{q}}
$$

In $L=T-V$ case $H=\frac{p^{2}}{2 m}+V(q)$ and Hamilton's equations of motion are-

$$
\dot{q}=\frac{\partial H}{\partial p}, \dot{p}=-\frac{\partial H}{\partial q}
$$

In special case, we obtain

$$
\dot{q}=\frac{p}{m}, \dot{p}=-\frac{d V}{d q}
$$

For a general observable-

$$
\dot{\mathcal{O}}=\frac{\partial \mathcal{O}}{\partial q} \dot{q}+\frac{\partial \mathcal{O}}{\partial p} \dot{p}=\frac{\partial \mathcal{O}}{\partial q} \frac{\partial H}{\partial p}-\frac{\partial \mathcal{O}}{\partial p} \frac{\partial H}{\partial q}
$$

Or this equation can be written as-

$$
\frac{d \mathcal{O}}{d t}=\{\mathcal{O}, H\}
$$

Here $\{A, B\}$ is position bracket acts as- 


$$
\{A, B\}=\frac{\partial A}{\partial q} \frac{\partial B}{\partial p}-\frac{\partial A}{\partial p} \frac{\partial B}{\partial q}
$$

Now in quantum mechanical sense, we write poison bracket as-

$$
\{A, B\} \rightarrow-i\{A, B\}
$$

For $\hbar=1$, we also have-

$$
[q, p]=i
$$

And

$$
\frac{d \mathcal{O}}{d t}=-i[\mathcal{O}, H]
$$

This is the Heisenberg equation for evolution of a quantum system. This equation has solution like this-

$$
\mathcal{O}(t)=e^{i H t} \mathcal{O}(0) e^{-i H t}
$$

Here $U(t)=e^{-i H t}$ is evolution operator.

Now how this equation can be defined in terms of Generalized Quantum Mechanics? The answer lies in my generalized mathematical relation for Uncertainty Principle. From equation (295), we can write-

$$
\Delta \Phi \Delta \psi \geq \frac{\Phi \alpha}{(\psi+\alpha \Phi)} \cdot \Gamma(\Phi) \cdot c \cdot \hbar_{x}
$$

We can also write this relation as-

$$
\begin{gathered}
\Delta \Phi \Delta \psi \geq \frac{c . \Gamma(\Phi)}{\left(1+\frac{\psi}{\Phi \alpha}\right)} \cdot \hbar_{x} \\
(\because 227) \\
\left\{\frac{\psi}{\Phi \alpha}=\beta\right\}
\end{gathered}
$$

So, we can write above equation as-

$$
\Delta \Phi \Delta \psi \geq \frac{c \cdot \Gamma(\Phi)}{(1+\beta)} \cdot \hbar_{x}
$$

Or, by putting $\frac{c \cdot \Gamma(\Phi)}{(1+\beta)}=\mathcal{T}_{h}$, we get above equation as-

$$
\Delta \phi \Delta \psi \geq \mathcal{T}_{h} \cdot \hbar_{x}
$$

From here we get two parameters as $\psi$ and $\phi$, as for a general observable-

$$
\dot{\mathcal{O}}=\frac{\partial \mathcal{O}}{\partial \phi} \dot{\phi}+\frac{\partial \mathcal{O}}{\partial \psi} \dot{\psi}=\frac{\partial \mathcal{O}}{\partial \phi} \frac{\partial \phi}{\partial \tau}+\frac{\partial \mathcal{O}}{\partial \psi} \frac{\partial \psi}{\partial \tau}
$$

$$
\begin{aligned}
\because E=\phi \psi \text { or } \frac{\partial E}{\partial \phi} & =\psi, \frac{\partial E}{\partial \psi} \\
& =\phi \text { for zero } \phi-\psi \text { Transformation }
\end{aligned}
$$

But by including $\phi-\psi$ transformation-

$$
\frac{\partial E}{\partial \phi}=\psi+\frac{\partial \psi}{\partial \phi} . \phi
$$

Or

$$
\frac{\partial E}{\partial \psi}=\phi+\frac{\partial \phi}{\partial \psi} \cdot \psi
$$

As we know for $\phi-\psi$ transformation- $\partial \psi=\alpha \partial \phi$. So, we can write both above equations as-

$$
\frac{\partial E}{\partial \phi}=\psi+\alpha \phi=\psi_{t}
$$

Or

$$
\frac{\partial E}{\partial \psi}=\phi+\frac{\psi}{\alpha}=\phi+\phi^{\prime}=\phi_{t}
$$

As we know now from my former paper [6] that-

$$
\psi_{t}=\alpha \phi_{t}
$$

So, we can write above equation as-

$$
\frac{\partial E}{\partial \phi}=\alpha \frac{\partial E}{\partial \psi}
$$


Now for conserved energy system, we know-

$$
\frac{\partial E}{\partial \phi}=\phi \frac{\partial \psi}{\partial \tau}+\psi \frac{\partial \phi}{\partial \tau}=0
$$

Or

$$
\frac{\partial \phi}{\partial \tau}=-\frac{\phi}{\psi} \cdot \frac{\partial \psi}{\partial \tau}
$$

Hence-

$$
\frac{1}{\phi} \frac{\partial \phi}{\partial \tau}=-\frac{1}{\psi} \cdot \frac{\partial \psi}{\partial \tau}
$$

For an observable $\mathcal{O}(\phi, \psi)$ we can write equation (530) as-

$$
\dot{\mathcal{O}}=-\frac{\partial \mathcal{O}}{\partial \phi}\left(\frac{\phi}{\psi}\right) \frac{\partial \psi}{\partial \tau}+\frac{\partial \mathcal{O}}{\partial \psi} \frac{\partial \psi}{\partial \tau}
$$

Or

$$
\dot{\mathcal{O}}=\frac{\partial \psi}{\partial \tau}\left(\frac{\partial \mathcal{O}}{\partial \psi}-\frac{\phi}{\psi} \cdot \frac{\partial \mathcal{O}}{\partial \phi}\right)
$$

Now by taking $\frac{1}{\psi}$ out from above equation, we get-

$$
\dot{\mathcal{O}}=\frac{1}{\psi} \frac{\partial \psi}{\partial \tau}\left(\psi \frac{\partial \mathcal{O}}{\partial \psi}-\phi \frac{\partial \mathcal{O}}{\partial \phi}\right)
$$

Now from equations (531) and (532), we get-

$$
\begin{gathered}
\psi=\frac{\partial E}{\partial \phi}-\alpha \phi=\frac{\partial E}{\partial \phi}-\psi^{\prime} \\
\phi=\frac{\partial E}{\partial \psi}-\frac{\psi}{\alpha}=\frac{\partial E}{\partial \psi}-\phi^{\prime}
\end{gathered}
$$

Now by putting both values of and from equations (544) and (543), we can write equation (542) as-

$$
\dot{\mathcal{O}}=\frac{1}{\psi} \frac{\partial \psi}{\partial \tau}\left(\frac{\partial E}{\partial \phi} \frac{\partial \mathcal{O}}{\partial \psi}-\frac{\partial E}{\partial \psi} \frac{\partial \mathcal{O}}{\partial \phi}+\phi^{\prime} \frac{\partial \mathcal{O}}{\partial \phi}-\psi^{\prime} \frac{\partial \mathcal{O}}{\partial \psi}\right)
$$

As we know poison bracket can be written as-

$$
\dot{\mathcal{O}}=\frac{1}{\psi} \frac{\partial \psi}{\partial \tau}\{\mathcal{O}, E\}+\frac{1}{\psi} \frac{\partial \psi}{\partial \tau}\left(\phi^{\prime} \frac{\partial \mathcal{O}}{\partial \phi}-\psi^{\prime} \frac{\partial \mathcal{O}}{\partial \psi}\right)
$$

Now by some manipulations in above equation, we get-

$$
\dot{\mathcal{O}}=\frac{1}{\psi} \frac{\partial \psi}{\partial \tau}\{\mathcal{O}, E\}+\frac{1}{\psi} \frac{\partial \psi}{\partial \tau}\left(\frac{\psi}{\alpha} \frac{\partial \mathcal{O}}{\partial \phi}-\alpha \phi \frac{\partial \mathcal{O}}{\partial \psi}\right)
$$

Or we can write it as-

$$
\dot{\mathcal{O}}=\frac{1}{\psi} \frac{\partial \psi}{\partial \tau}\{\mathcal{O}, E\}+\left(\frac{1}{\psi} \frac{\partial \psi}{\partial \tau} \frac{\psi}{\alpha} \frac{\partial \mathcal{O}}{\partial \phi}-\frac{\alpha \phi}{\psi} \frac{\partial \psi}{\partial \tau} \frac{\partial \mathcal{O}}{\partial \psi}\right)
$$

$$
\begin{gathered}
\{\because \partial \psi=\alpha \partial \phi\} \\
\dot{\mathcal{O}}=\frac{1}{\psi} \frac{\partial \psi}{\partial \tau}\{\mathcal{O}, E\}+\left(\frac{\partial \psi}{\alpha \partial \phi} \frac{\psi}{\psi} \frac{\partial \mathcal{O}}{\partial \tau}-\frac{\alpha \phi}{\psi} \frac{\partial \psi}{\partial \psi} \frac{\partial \mathcal{O}}{\partial \tau}\right)
\end{gathered}
$$

$$
\begin{aligned}
\dot{\mathcal{O}}=\frac{1}{\psi} \frac{\partial \psi}{\partial \tau}\{\mathcal{O}, E\} & +\frac{\partial \mathcal{O}}{\partial \tau}\left(1-\frac{\alpha \phi}{\psi}\right) \Rightarrow \dot{\mathcal{O}}\left(1-1+\frac{\alpha \phi}{\psi}\right) \\
& =\frac{1}{\psi} \frac{\partial \psi}{\partial \tau}\{\mathcal{O}, E\}
\end{aligned}
$$

For a system as we know that-

$$
\dot{\mathcal{O}}=\frac{1}{\phi} \frac{\partial \phi}{\partial \tau}\{\mathcal{O}, E\}=\frac{1}{\psi^{\prime}} \frac{\partial \psi}{\partial \tau}\{\mathcal{O}, E\}
$$

(551)

This equation is valid for Quantum Mechanics in all cases for duality and Classical Mechanics also without including duality. In this case evolution of operators will be like-

$$
\mathcal{O}(t)=e^{\left(\frac{\dot{\phi}}{\phi}\right) E \tau} \mathcal{O}(0) e^{-\left(\frac{\dot{\phi}}{\phi}\right) E \tau}
$$

\subsection{Justification of Old Quantum Theory and Planck's Distribution}

Now I intend to specify the pretty beginning of Quantum Mechanics from Planck's law of black body radiation 8 and how this law makes a particular sense in my theoretical perspective. Now from Stephens's law 39 we get the total intensity (or the total power per unit surface area) radiated by a glowing object of temperature $T$ is given by- 


$$
P=a \sigma T^{4}
$$

This is known as Stefan Boltzmann law [39] and here $\sigma=$ $5.67 \times 10^{-8} \mathrm{Wm}^{-2} \mathrm{~K}^{-4}$ is Stefan Boltzmann constant ora is coefficient $a \in[0,1]$ and for black body $a=1$ and distribution is represented as-

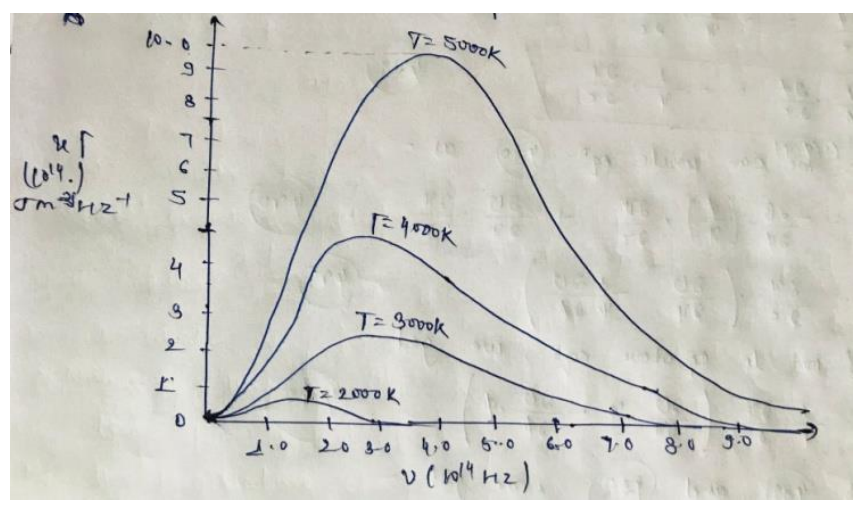

FIG. 13 Variation in Energy Density of Radiation of Blackbody with Respect to Temperature

And the approach of Rayleigh-Jeans distribution, Wien's distribution and Planck's distribution is like this-

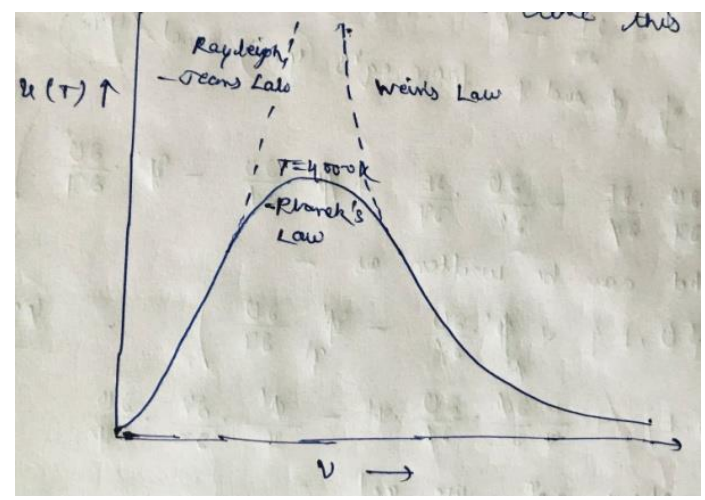

FIG. 14 Rayleigh-Jeans distribution, Wien's distribution and Planck's distribution

At first by using some thermodynamics arguments, Wien [41] took the Stefan- Boltzmann low equation (552) and in 1894 he just obtained the energy density per unit frequency of the emitted black body radiation as-

$$
u(v, T)=A v^{3} e^{-\beta v / T}
$$

\section{Here $A$ and $\beta$ are some parameters.}

At that time in 1900 Rayleigh [42] attempted his distribution in terms of number of modes (standing electromagnetic waves) of radiation in the frequency interval $v$ to $v+d v$ as-

$$
N(v)=\frac{8 \pi v^{3}}{c^{3}}
$$

Here $c=3 \times 10^{8}$ is speed of light.

So, energy density is given by-

$$
u(v, T)=N(v)\langle E\rangle=\frac{8 \pi v^{2}}{c^{3}}\langle E\rangle
$$

Here $\langle E\rangle$ is the average energy of oscillator. Now according to equipartition theorem, all oscillators in cavity have the same mean energy, irrespective of their frequencies-

$$
\langle E\rangle=\frac{\int_{0}^{\infty} E \cdot e^{-\left(E / k_{B} T\right)} \cdot d E}{\int_{0}^{\infty} e^{-\left(E / k_{B} T\right)} \cdot d E}=k_{B} T
$$

Here $k_{B}=1.3807 \times 10^{-23} J^{-1}$ is the Boltzmann constant.

Now by putting value in equation (555), we get-

$$
u(v, T)=\frac{8 \pi v^{2}}{c^{3}} k_{B} T
$$

This is the Rayleigh-jeans distribution. This gives rise to a very classical problem ultraviolet catastrophe which can't be solved by Classical Mechanics. After that in particular year 1901 Max Planck [43] used both Wien's and Rayleigh-jeans distributions to solve ultraviolet catastrophe and proposed an accurate description of black body radiation. Planck considered that the energy exchange between radiation and matter must be discreet. He then postulated that energy of radiation (of frequency $v$ ) emitted by the oscillating charges must come in only integer multiples of $h v$ as-

$$
E=n h v
$$

\section{(558)}

Here is universal constant of Planck and is quantum of radiation. Plank obtain mean energy as-

$$
\langle E\rangle=\frac{\int_{0}^{\infty} n h v \cdot e^{-\left(n h v / k_{B} T\right)} \cdot d E}{\int_{0}^{\infty} e^{-\left(n h v / k_{B} T\right)} \cdot d E}=\frac{h v}{e^{h v / k_{B} T}-1}
$$

Or the density distribution by Planck can be obtained by using equation (555) as-

$$
u(v, T)=\frac{8 \pi v^{2}}{c^{3}} \frac{h v}{e^{h v / k_{B} T}-1}
$$


This is Planck's distribution. Here- $h=6.626 \times 10^{-34} J-$ sec

In terms of frequency to wavelength we can write it as-

$$
\tilde{u}(\lambda, T)=\frac{8 \pi h c}{\lambda^{5}} \frac{1}{e^{h c / \lambda k_{B} T}-1}
$$

Now by doing integration of $u(v, T)$ over $d v$ from $(0, \infty)$, we get-

$$
\begin{aligned}
\int_{0}^{\infty} u(v, T)=\frac{8 \pi h}{c^{3}} \int_{0}^{\infty} \frac{v^{3}}{e^{h v / k_{B} T}-1} d v \\
\quad=\frac{8 \pi k_{B}^{4} T^{4}}{h^{3} c^{3}} \int_{0}^{\infty} \frac{x^{3}}{e^{x}-1} d v=\frac{8 \pi k_{B}^{4} T^{4}}{h^{3} c^{3}} \\
=\frac{4}{c} \sigma T^{4}
\end{aligned}
$$

Now if we replace $h$ by $h_{x}$ and $T$ by quantity of disturbance $H^{0}$, for N-Central Systems, we get-

$$
P=\sigma_{x}\left(H^{0}\right)^{4}
$$

And extended Planck's distribution in Generalized Quantum Mechanics as-

$$
u\left(v, H^{0}\right)=\frac{8 \pi v^{2}}{c^{3}} \frac{h_{x} v}{e^{\left(\frac{h_{x} v}{k_{B}^{\prime} H^{0}}\right)}-1}
$$

Here from my former paper [5], quantity of disturbance is-

$$
H^{0}=\mathrm{p} \frac{\Delta F_{s} \cdot \Delta \alpha_{s}}{\eta_{s} \cdot \Delta \Phi_{s}}
$$

For broken parts-

$$
E_{b}=\phi_{b} \psi_{b}=h_{x} v
$$

Or

$$
E_{c}=\alpha \phi \Delta \phi=n E_{b}=n h_{x} v
$$

We can also get value of $h_{x}$ from energy of broken parts as-

$$
h_{x}=\frac{\phi_{b} \psi_{b}}{v}
$$

Now by putting value of and from equations (567) and (566) in equation (564), we get-

$$
u\left(v, H^{0}\right)=\frac{8 \pi \phi_{b}^{3} \psi_{b}^{3}}{h_{x}^{2}\left(v_{p}\right)_{b}^{3}} \frac{1}{e^{\left(\frac{\phi_{b} \psi_{b} \eta_{s} . \Delta \Phi_{s}}{\mathrm{p} k_{B}^{\prime} \Delta F_{s} \cdot \Delta \alpha_{s}}\right)}-1}
$$

Now by solving it further, we can write it as-

$$
u\left(\phi_{b}, \psi_{b}\right)=\frac{8 \pi}{h_{x}^{2}}\left(\frac{\phi_{b} \psi_{b}}{\left(v_{p}\right)_{b}}\right)^{3} \frac{1}{e^{\left(\frac{\phi_{b} \psi_{b} \eta_{s} . \Delta \Phi_{s}}{\mathrm{p} k_{B}^{\prime} \Delta F_{s} \cdot \Delta \alpha_{s}}\right)}-1}
$$

Now by putting $\theta_{b}=\frac{\phi_{b} \psi_{b}}{\mathrm{p} k_{B}^{\prime}} \frac{\eta_{s} \cdot \Delta \Phi_{s}}{\Delta F_{s} \cdot \Delta \alpha_{s}}$, we get-

$$
u\left(\phi_{b}, \psi_{b}\right)=\frac{8 \pi}{h_{x}^{2}}\left(\frac{\phi_{b} \psi_{b}}{\left(v_{p}\right)_{b}}\right)^{3} \frac{1}{e^{\theta_{b}}-1}
$$

This distribution is valid for all type of broken parts exists in Universe. For N-Central Systems, we can write StefanBoltzmann low as by putting value of $H^{0}$ from equation (565) to equation (563), as-

$$
P_{x}=\sigma_{x}\left(\mathrm{p}_{x} \frac{\Delta F_{s} \cdot \Delta \alpha_{s}}{\eta_{s} \cdot \Delta \Phi_{s}}\right)^{4}
$$

Or for small variation, we can write it as-

$$
P_{x}=\sigma_{x} \mathrm{p}_{x}^{4} \frac{\left(\Delta F_{s}\right)^{4}}{\eta_{s}}\left(\frac{d \alpha_{s}}{d \Phi_{s}}\right)^{4}
$$

So, this equation is extended Stefan-Boltzmann law in universal sense and $x$ represents the type of Central System here.

Now in Einstein's terms we can write energy of a broken part as-

$$
E_{b}=\psi_{b}\left(v_{p}\right)_{b}^{2}
$$


Now from equation (568), we can write $\psi_{b}=\frac{h_{x} v}{\phi_{b}}$. So, above equation becomes-

$$
E_{b}=\frac{h_{x} v}{\phi_{b}}\left(v_{p}\right)_{b}^{2}
$$

This equation holds only if-

$$
\left(v_{p}\right)_{b}^{2}=\phi_{b}
$$

Here $E_{b}=h_{x} v$.

Therefore-

$$
\left(v_{p}\right)_{b}=\left(\phi_{b}\right)^{1 / 2}
$$

Now we have defined at least everything fundamental in Quantum Mechanics and General Relativity from only one theoretical perspective over by using the Principle of Central System Relativity. Here only two things remains in Quantum Mechanics which are very fundamental in their own sense, known as And Earnfest theorem [44] Which will be proven in a separate paper and Pauli's Spin Statistics Theorem [45] predicted distributions known as Fermi-Dirac distribution [46] and Bose-Einstein distribution [47].

\subsection{Pauli's Spin Statistics Theorem in Universal Sense with Fermi-Dirac Distribution and Bose-Einstein Distribution}

So, at first I intend to define Spin Statistics Theorem in terms of my theoretical perspective and after that I will go through these both kinds of distributions linked with this particular theorem. This theorem predicts two types of particles (or bodies) as integer spin particles (bosons or broken parts) and half integer spin particles (fermions or normal perfect and imperfect bodies). Now this controversy can be solved by looking at the configuration of these particles or bodies (defined by all kind of observers in central relativistic sense) that I defined configuration of broken parts as they rotate both sides in scalar field. So, these kind of broken parts always have spin in full integral but perfect or imperfect bodies usually have their spin in a particular direction. So, these kinds of bodies have half integral spin. We can also represent these both kinds of bodies in geometrical way, as-

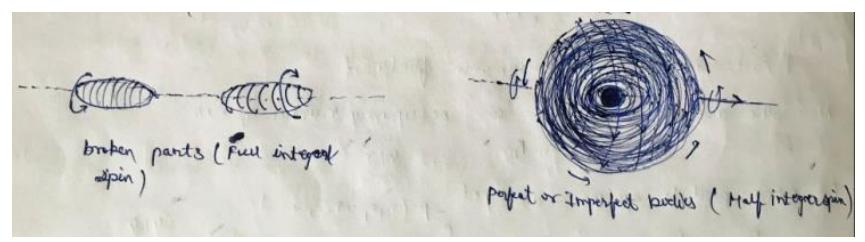

FIG. 15 Geometrical Representation of Comparison of Propagation style of Broken Part and Body in Scalar Field

So, in this way we can define these both kind of bodies and broken parts carries spin respectively half and full integral spin. Now this perspective holds for all kinds of bodies and broken parts exist in Universe period now let's go to the statement of Spin Statistics Theorem. Pauli defined this Spin Statistics Theorem as "The fields of integral spins commute (and therefore must be quantized as Bosons) while the fields of half integral spins anti-commute (and therefore must be quantized as fermions)". Or in mathematical way we can represent it as-

$$
\hat{\phi}_{A}(x) \hat{\phi}_{B}^{\dagger}(y)=\hat{\phi}_{B}^{\dagger}(y) \hat{\phi}_{A}(x)-\text { For Bosonic Fields }
$$

And

$$
\hat{\phi}_{A}(x) \hat{\phi}_{B}^{\dagger}(y)=-\hat{\phi}_{B}^{\dagger}(y) \hat{\phi}_{A}(x)-\text { For Fermionic Fields }
$$

Now in my former paper [6], I obtained some relations like this. Or in other words, we can state above from as- "The wave function of a system of identical integral spin particles has the same value when the positions of any two particles are swapped. These particles are known as both phones over the wave function of a system of identical half integer spin particles change sign when two particles are swapped and these particles with wave function anti-symmetric under exchange are known as fermions".

The average number of fermions in a single particle state is given by Fermi-Dirac distribution, as-

$$
\bar{n}_{i}=\frac{1}{e^{\frac{\left(E_{i}-\mu\right)}{k_{B} T}}+1}
$$

Or for bosons (integral spin particles) average number of particles is given by Bose-Einstein distribution as-

$$
\bar{n}_{i}=\frac{1}{e^{\frac{\left(E_{i}-\mu\right)}{k_{B} T}}-1}
$$


Now let's have a look on the derivation of Fermi-Dirac distribution. For $\mathrm{N}$ identical fermions that have negligible mutual interaction and are in thermal equilibrium, then-

$$
E_{R}=\sum_{r} n_{r} E_{r}
$$

Here $n_{r}$ is the occupation number of single particle state with energy $E_{r}$.

The probability in state are can be defined by normalized canonical distribution [48] as-

$$
P_{R}=\frac{e^{-\beta E_{R}}}{\sum_{R^{\prime}} e^{-\beta E_{R^{\prime}}}}
$$

Here $\beta=\frac{1}{k_{B} T}$ and $e^{-\beta E_{R}}$ is Boltzmann factor.

Now the average value for an occupancy number $n_{i}$ is-

$$
\bar{n}_{i}=\sum_{R} n_{i} P_{R}
$$

For many particle systems, we get-

$$
P_{R}=P_{n_{1}, n_{2}, \ldots .}=\frac{e^{-\beta\left(n_{1} E_{1}+n_{2} E_{2}+\cdots\right)}}{\sum_{n_{1}^{\prime}, n_{2 . .}^{\prime}} e^{-\beta\left(n_{1}^{\prime} E_{1}+n_{2}^{\prime} E_{2}+\cdots\right)}}
$$

Now $\bar{n}_{i}$ becomes-

$$
\bar{n}_{i}=\sum_{n_{1}, n_{2 .}} n_{i} P_{n_{1}, n_{2}, \ldots .}=\frac{\sum_{n_{1}, n_{2 . .}} n_{i} e^{-\beta\left(n_{1} E_{1}+n_{2} E_{2}+\cdots+n_{i} E_{i \cdot .}\right)}}{\sum_{n_{1}, n_{2 . .}} e^{-\beta\left(n_{1} E_{1}+n_{2} E_{2}+\cdots+n_{i} E_{i \cdot .}\right)}}
$$

Now by Pauli Exclusion Principle [49], $n_{r}=0$ or 1 for each $r$ and total number of particles is N. so-

$$
\sum_{r} n_{r}=N
$$

Now by rearranging the summation, we get-

$$
\bar{n}_{i}=\frac{\sum_{n_{i}=0}^{1} n_{i} e^{-\beta\left(n_{i} E_{i}\right)}+\sum_{n_{1}, n_{2 . .}}^{(i)} e^{-\beta\left(n_{1} E_{1}+n_{2} E_{2}+\cdots+n_{i} E_{i . .}\right)}}{\sum_{n_{i}=0}^{1} e^{-\beta\left(n_{i} E_{i}\right)}+\sum_{n_{1}, n_{2 . .}}^{(i)} e^{-\beta\left(n_{1} E_{1}+n_{2} E_{2}+\cdots+n_{i} E_{i . .}\right)}}
$$

Here $(i)$ indicate summation is not over $n_{i}$ and is $N_{i}=N-$ $n_{i}$. In one case $n_{i}=0$ and $\sum_{. .}^{(i)}$ is evolution with $N_{i}=N$, but in $\sum_{. .}^{(i)}$ is evaluated with $N_{i}=N-1$. So, we can write this sum as-

$$
Z_{i}\left(N-n_{i}\right) \equiv \sum_{n_{1}, n_{2 \cdot .}}^{(i)} e^{-\beta\left(n_{1} E_{1}+n_{2} E_{2}+\cdots+n_{i} E_{i \cdot .}\right)}
$$

So, by putting equation (589) we get equation (588) as-

$$
\begin{aligned}
\bar{n}_{i}=\frac{\sum_{n_{i}=0}^{1} n_{i} e^{-\beta\left(n_{i} E_{i}\right)}+Z_{i}\left(N-n_{i}\right)}{\sum_{n_{i}=0}^{1} e^{-\beta\left(n_{i} E_{i}\right)}+Z_{i}\left(N-n_{i}\right)} \\
=\frac{0+e^{-\beta\left(n_{i} E_{i}\right)} Z_{i}(N-1)}{e^{-\beta\left(n_{i} E_{i}\right)} Z_{i}(N-1)+Z_{i}(N)} \\
=\frac{1}{\left[\frac{Z_{i}(N)}{Z_{i}(N-1)}\right] e^{\beta E_{i}+1}}
\end{aligned}
$$

Now for $\frac{Z_{i}(N)}{Z_{i}(N-1)}$, we get an approximation as-

$$
\ln Z_{i}(N-1) \approx \ln Z_{i}(N)-\frac{\partial \ln Z_{i}(N)}{\partial N}=\ln Z_{i}(N)-\alpha_{i}
$$

$$
\left\{H \text { ere } \alpha_{i}=\frac{\partial \ln Z_{i}(N)}{\partial N}\right\}
$$

Now if number of particles $\mathrm{N}$ is large enough so that the change in chemical potential $\mu$ is very small, then $\alpha_{i} \cong$ $-\mu / k_{B} T$. So, we get $\alpha_{i}$ value as-

$$
\frac{Z_{i}(N)}{Z_{i}(N-1)}=e^{-\frac{\mu}{k_{B} T}}
$$

Now by putting this value in equation (590), we get-

$$
\bar{n}_{i}=\frac{1}{e^{\frac{\left(E_{i}-\mu\right)}{k_{B} T}}+1}
$$

In similar way Bose-Einstein distribution [47] can be obtained for integral spin particles by using canonical distribution [48]. Now suppose an observer made of solar systems (like we made of atoms) observes planets from Galaxy level, Than according to him laws of physics will be similar a Generalized Quantum Mechanics and after that he will observe continuous properties of matter like we cannot see $(\mathrm{k}-2)^{\mathrm{th}}$ bodies or shorter bodies from which the electrons orders that subatomic 
particles are made of. So, Generalized Quantum Mechanics works for all such observers who are looking for physics laws from $\mathrm{k}^{\text {th }}$ Central Systems to $(\mathrm{k}-1)^{\text {th }}$ Central Systems. Now come to the derivation of Bose-Einstein distribution as-

$$
W_{n_{1}, n_{2}, \ldots n_{i}, . n_{k}}=\prod \frac{\left(n_{i}+g_{i}\right) !}{\left(n_{i}\right) !\left(g_{i}\right) !}
$$

Here $W_{i}$ is the actual number of ways in which $n_{i}$ particles are to be distributed in $g_{i}$ cells in the $\mathrm{i}^{\text {th }}$ compartment as-

$$
W_{i}=\frac{\left(n_{i}+g_{i}-1\right) !}{\left(n_{i}\right) !\left(g_{i}-1\right) !}
$$

Now taking logarithm of equation (590) for both sides, we get-

$$
\ln W=\sum_{i=1}^{N}\left[\ln \left(n_{i}+g_{i}\right) !-\ln \left(n_{i}\right) !-\ln \left(g_{i}\right) !\right]
$$

For large $n_{i}$ and $g_{i}$, we get by Stirling Approximation as-

$$
\ln n !=n \ln n-n
$$

So, now by applying this approximation to equation (596), we get-

$$
\begin{aligned}
\ln W=\sum_{i=1}^{k}\left[\left(n_{i}+\right.\right. & \left.g_{i}\right) \ln \left(n_{i}+g_{i}\right)-\left(n_{i}+g_{i}\right) \\
& \left.-n_{i} \ln \left(n_{i}\right)+n_{i}-g_{i} \ln \left(g_{i}\right)+g_{i}\right]
\end{aligned}
$$

For a state of maximum thermodynamic probability, we get-

$$
\delta(\ln W)=0
$$

So, we get by putting value of from equation (598) in above equation as-

$$
\begin{gathered}
\delta(\ln W)=\sum_{i=1}^{k}\left[\delta n_{i} \ln \left(n_{i}+g_{i}\right)-\left(n_{i}+g_{i}\right) \frac{1}{\left(n_{i}+g_{i}\right)} \delta\left(n_{i}\right)\right. \\
\left.-\delta n_{i} \ln \left(n_{i}\right)+\delta n_{i}\right] \\
=\sum_{i=1}^{k}\left[\delta n_{i} \ln \left(n_{i}+g_{i}\right)-\delta n_{i} \ln \left(n_{i}\right)\right]
\end{gathered}
$$

$$
\left\{\text { Here } \delta g_{i}=0\right\}
$$

Or

$$
\begin{aligned}
\delta(\ln W)=\sum_{i=1}^{k}\left[\ln \frac{\left(n_{i}+g_{i}\right)}{n_{i}}\right] \delta n_{i} \\
\quad=-\sum_{i=1}^{k}\left[\ln \frac{n_{i}}{\left(n_{i}+g_{i}\right)}\right] \delta n_{i}=0
\end{aligned}
$$

Or

$$
\sum_{i=1}^{k}\left[\ln \frac{n_{i}}{\left(n_{i}+g_{i}\right)}\right] \delta n_{i}=0
$$

Now for bosons-

$$
n=\sum_{i} n_{i}=\text { Constant }
$$

$$
\delta n=\sum_{i} \delta n_{i}=0
$$

For conservation of energy, we can write-

$$
E=\sum_{i} n_{i} E_{i}=\text { Constant }
$$

Or

$$
\delta E=\sum_{i}\left[E_{i}\right] \delta n_{i}=0
$$

Now we get-

$$
\delta E=\sum_{i}\left[\ln \frac{n_{i}}{\left(n_{i}+g_{i}\right)}+\alpha+\beta E_{i}\right] \delta n_{i}=0
$$

So, we get- 


$$
\ln \frac{n_{i}}{\left(n_{i}+g_{i}\right)}+\alpha+\beta E_{i}=0
$$

Or

$$
\ln \frac{n_{i}}{\left(n_{i}+g_{i}\right)}=-\alpha-\beta E_{i}
$$

Now by taking antilogarithm both sides to equation (609), we get-

$$
\frac{n_{i}}{\left(n_{i}+g_{i}\right)}=e^{-\left(\alpha+\beta E_{i}\right)}
$$

Or

$$
\frac{\left(n_{i}+g_{i}\right)}{n_{i}}=1+\frac{g_{i}}{n_{i}}=e^{\left(\alpha+\beta E_{i}\right)}
$$

So, we get-

$$
\frac{g_{i}}{n_{i}}=e^{\left(\alpha+\beta E_{i}\right)}-1
$$

Or

$$
n_{i}=\frac{g_{i}}{e^{\left(\alpha+\beta E_{i}\right)}-1}
$$

So, this is the expression for Bose-Einstein distribution. Now in both different cases the usual difference in terms of universal mechanic is for bosons $E_{i}=\left(\phi_{b} \psi_{b}\right)_{i}$ or for fermions $E_{i}=(\phi \psi)_{i}$ where $\psi_{p} \neq 0$. So, we can write both distributions as-

$$
n_{i}=\frac{g_{i}}{e^{\left(\frac{\phi_{b} \psi_{b}-\mu}{k_{B} H^{0}}\right)}-1}
$$

Or

$$
n_{i}=\frac{g_{i}}{e^{\left(\frac{\phi \psi-\mu}{k_{B} H^{0}}\right)}+1}
$$

Here $\mu$ is mixed energy of particles generated by mixing of their scalar fields. So, Generalized Bose-Einstein and Fermi-
Dirac distributions can be defined in Generalized Quantum Mechanics as-

$$
n_{i}=\frac{g_{i}}{e^{\left(\frac{\left(\phi_{b} \psi_{b}-\mu\right) \eta_{s} \cdot \Delta \Phi_{s}}{\mathrm{p} k_{B}^{\prime} \Delta F_{s} \cdot \Delta \alpha_{s}}\right)}-1}
$$

Or

$$
n_{i}=\frac{g_{i}}{e^{\left(\frac{(\phi \psi-\mu) \eta_{s} \Delta \Phi_{s}}{\mathrm{p} k_{B}^{\prime} \Delta F_{s} \cdot \Delta \alpha_{s}}\right)}+1}
$$

Now come at Spin Statistics Theorem [45] in Generalized various or also to the Pauli's exclusion Principle [49].

The wave function of bosons commute due to $\psi_{p}=0$ for broken parts (bosons) but the wave function of fermions anti commute because $\psi_{p} \neq 0$ or perfection quantity for fermions is not zero. As we know Spin Statistics Theorem and Pauli Exclusion Principle both are depend upon the particular phenomenology. So, we can generalize both theorems and Principles as- "The body is for which perfection quantity $\psi_{p}$ is zero commute and follow a separate statistics rather than the bodies which have same non-transformable and perfection quantity $\psi_{p} \neq 0$ or their wave functions don't commute or anti commute". We can represent this phenomenology in mathematical way (for $\psi_{p}=0$, we can say broken parts) as-

$$
\left\{\mathcal{P}_{b}(x), \mathcal{P}_{b}(y)\right\}=\mathcal{P}_{b}(x) \mathcal{P}_{b}(y)-\mathcal{P}_{b}(y) \mathcal{P}_{b}(x)=0
$$

Or for $\psi_{p} \neq 0$ or normal bodies-

$$
\{\mathcal{P}(x), \mathcal{P}(y)\}=\mathcal{P}(x) \mathcal{P}(y)+\mathcal{P}(y) \mathcal{P}(x)=0
$$

In other words, we can say $\psi_{p}=0$ bodies (broken parts) have integer spin but $\psi_{p} \neq 0$ bodies (perfect or imperfect bodies) have half integral spin.

Or-

$$
\mathcal{P}(x, y)=\mathcal{P}(y, x) \text { for } \psi_{p}=0
$$

And

$$
\mathcal{P}(x, y)=-\mathcal{P}(y, x) \text { for } \psi_{p} \neq 0
$$




$$
\mathcal{P}(x) \mathcal{P}(y)=\mathcal{P}(y) \mathcal{P}(x) \text { for } \psi_{p}=0
$$

And

$$
\mathcal{P}(x) \mathcal{P}(y)=-\mathcal{P}(y) \mathcal{P}(x) \text { for } \psi_{p} \neq 0
$$

In another way we can also start that fully transformable bodies $\psi_{p}=0$ for broken parts have spin in both directions of its propagating axis but non-transformable bodies $\psi_{p} \neq 0$ have spin in a particular direction. So, this particular can't be occupied by other body without collapsing. As I defined earlier that $\psi_{p} \neq 0$ bodies follows different statistics. Expressed in Generalized Quantum Mechanics as-

$$
n_{i}=\frac{g_{i}}{e^{\left(\frac{(\phi \psi-\mu) \eta_{S} \cdot \Delta \Phi_{S}}{\mathrm{p} k_{B}^{\prime} \Delta F_{S} \cdot \Delta \alpha_{S}}\right)}+1}
$$

Or for bodies follow the symmetric wave function properties with $\psi_{p}=0$ or broken parts have the statistics as-

$$
n_{i}=\frac{g_{i}}{e^{\left(\frac{\left(\phi_{b} \psi_{b}-\mu\right) \eta_{s} \cdot \Delta \Phi_{s}}{\mathrm{p} k_{B}^{\prime} \Delta F_{s} \cdot \Delta \alpha_{s}}\right)}-1}
$$

So, in this way we can define particles (in terms of Generalized Quantum Mechanical view) or their statistics related to their way of propagating in scalar field.

\section{PROPAGATION SPEED OF WAVES IN SCALAR FIELD DENSITY}

Now I am defining a very elegant property of Universe in terms of the speed of propagation of waves through any scalar field $\phi$ having density $\rho_{\phi}$. Formerly this kind of waves are defined as gravitational waves as an outstanding prediction of theory of General Relativity and proven by Kip Throne [51] in 2017 (after 100 years of this theoretical prediction). So, at first I am starting all this with the former way of defining the speed of sound [50] in a medium of density $\rho$ following continuity equation. Now in the end of article, I am deriving an expression for velocity of propagation of waves in scalar field density $\rho_{\phi}$ as Sir Isaac Newton derived for sound in a medium [50]. Let's say velocity of propagation wave in scalar field of density $\rho_{\phi}$ is $\left(v_{\phi}\right)_{\omega}$ and $\left(v_{\phi}\right)_{\omega}$ can be written as-

$$
\left(v_{\phi}\right)_{\omega}=v \lambda
$$

Here $v$ is frequency of wave and $\lambda$ is wavelength of wave. Now a wave also satisfies wave equation as-

$$
\frac{\partial^{2} y(s, \tau)}{\partial s^{2}}=\frac{1}{\left(v_{\phi}\right)_{\omega}^{2}} \frac{\partial^{2} y(s, \tau)}{\partial \tau^{2}}
$$

Now suppose a scalar field symmetrically in a pipe of volume $\mathcal{V}$ as-

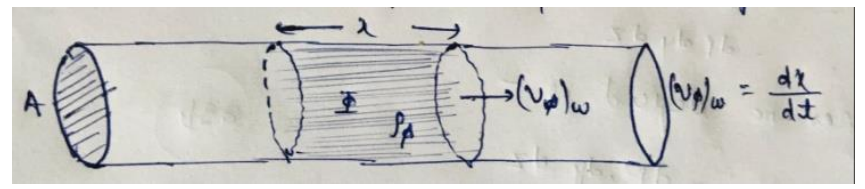

FIG. 16 Representation of Wave in Pipe filled with Scalar Field

$$
\Phi=\rho_{\phi} \mathcal{V}=\rho_{\phi} A \cdot x
$$

$$
\frac{\partial \Phi}{\partial \tau}=\rho_{\phi} A \frac{\partial x}{\partial \tau}=\rho_{\phi} A\left(v_{\phi}\right)_{\omega}
$$

Here we define constant scalar field density. So $\frac{\partial \rho_{\phi}}{\partial \tau}=0$, but in case of variation force we also take into account the differentiation $\frac{\partial \rho_{\phi}}{\partial \tau} \neq 0$. Now consider a wave moving through a parcel of scalar field $\Phi$. This parcel has a small volume of scalar field as-

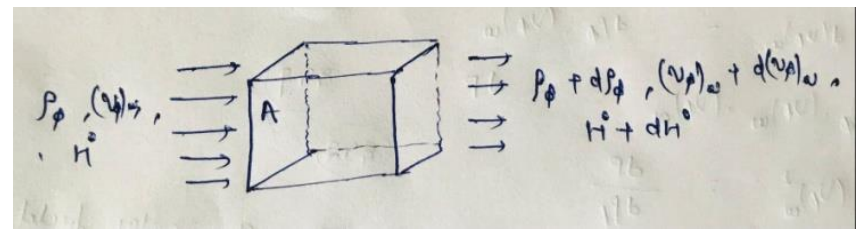

FIG. 17 Geometrical Representation of Variation in Pressure and Velocity Difference carried by Propagating Wave Through it

Here $H^{0}$ is quantity of disturbance.

Now the continuity equation states that the scalar field flow rate entering the volume is equal to the is scalar field flow rate leaving the volume so-

$$
\left(\rho_{\phi}+d \rho_{\phi}\right) A\left(\left(v_{\phi}\right)_{\omega}+d\left(v_{\phi}\right)_{\omega}\right)=\rho_{\phi} A\left(v_{\phi}\right)_{\omega}
$$

These changes are infinitesimal or $d \rho_{\phi} d\left(v_{\phi}\right)_{\omega} \approx 0$. 


$$
\begin{gathered}
\rho_{\phi}\left(v_{\phi}\right)_{\omega}=\left(\rho_{\phi}+d \rho_{\phi}\right)\left(\left(v_{\phi}\right)_{\omega}+d\left(v_{\phi}\right)_{\omega}\right) \\
\Rightarrow \rho_{\phi}\left(v_{\phi}\right)_{\omega}=\rho_{\phi}\left(v_{\phi}\right)_{\omega}+\rho_{\phi} d\left(v_{\phi}\right)_{\omega}+d \rho_{\phi}\left(v_{\phi}\right)_{\omega} \\
+\left(d \rho_{\phi}\right)\left(d\left(v_{\phi}\right)_{\omega}\right) \\
(632) \\
\Rightarrow \rho_{\phi} d\left(v_{\phi}\right)_{\omega}+d \rho_{\phi}\left(v_{\phi}\right)_{\omega}=0
\end{gathered}
$$

$$
\Rightarrow \rho_{\phi} d\left(v_{\phi}\right)_{\omega}=-d \rho_{\phi}\left(v_{\phi}\right)_{\omega}
$$

Now the net force on the volume of fluid is equal to the sum of the forces on the left face and the right face or represented as-

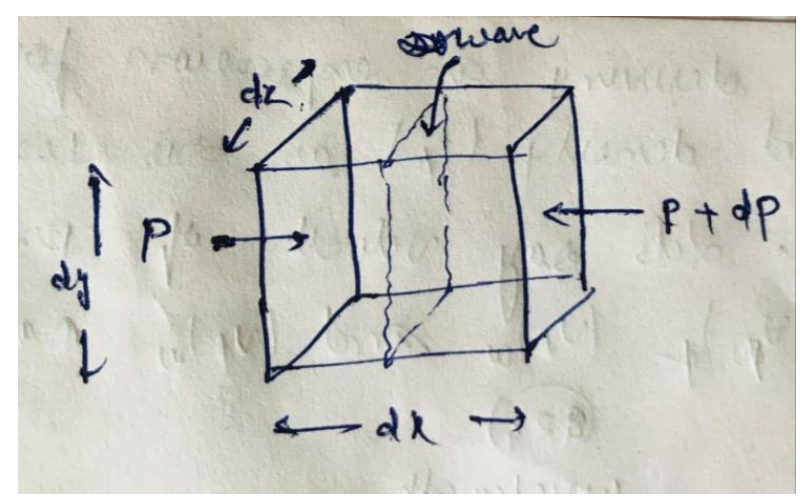

FIG. 18 Geometrical Representation of Variation in Pressure and Velocity Difference across Infinitesimal Volume Element

$$
\delta_{n e t}=P d y d z-(P+d P) d y d z
$$

$$
\because\{\delta=P . A\}
$$

Here $P$ is pressure o n scalar field.

From here we get-

$$
\delta_{n e t}=P d y d z-P d y d z-(d P) d y d z=-d P d y d z
$$

Now we know force can be defined as-

$$
\Phi \frac{d\left(v_{\phi}\right)_{\omega}}{d \tau}=-d P d y d z
$$

Or

Or we get-

We get-

Or

$$
d\left(v_{\phi}\right)_{\omega}=-\frac{d P}{\rho_{\phi} d x} d \tau=\frac{-d P}{\rho_{\phi}\left(\frac{d x}{d \tau}\right)}=\frac{-d P}{\rho_{\phi}\left(v_{\phi}\right)_{\omega}}
$$

Or we can also write above equation as-

$$
\rho_{\phi}\left(v_{\phi}\right)_{\omega} d\left(v_{\phi}\right)_{\omega}=-d P
$$

Now as we know from equation (634), that-

$$
\rho_{\phi} d\left(v_{\phi}\right)_{\omega}=-d \rho_{\phi}\left(v_{\phi}\right)_{\omega}
$$

$$
-d \rho_{\phi}\left(v_{\phi}\right)_{\omega}\left(v_{\phi}\right)_{\omega}=-d P
$$

$$
\left(v_{\phi}\right)_{\omega}^{2}=\frac{d P}{d \rho_{\phi}}
$$

So, we get velocity of propagation of a wave through a scalar field $\Phi$ of density $\rho_{\phi}$ as-

$$
\left(v_{\phi}\right)_{\omega}=\sqrt{\frac{d P}{d \rho_{\phi}}}
$$

Here pressure $P$ can be written as-

$$
P=\frac{\delta}{A}=\frac{\Phi}{A} \frac{d\left(v_{\phi}\right)_{\omega}}{d \tau}=\sigma_{\phi} \cdot \frac{d\left(v_{\phi}\right)_{\omega}}{d \tau}
$$

So, at different scalar field density is propagation wave through a particular scalar field is different or this also happens in terms of gravitational waves. In other words we can state that gravitational waves (predicted by theory of General Relativity) don't propagate at a constant (speed of 
light $c$ ) or it is also proven formerly by me that speed of light is not a constant in different scalar field density. So, Albert Einstein was quite wrong about the speed of gravitational waves and speed of light.

\section{NIFIED EQUATIONS OF BOTH THEORETICAL STRUCTURES}

So, in this particular article we have known how to unify both kinds of theories: microscopic (Quantum Mechanics) and macroscopic (General Relativity) in a single theoretical perspective (for sake of simplicity we can say it as "Universal Mechanics"). Here Universal Mechanics includes some special and unique laws governed by Universe as Central System Relativity, $\phi-\psi$ transformation, inflations, Universal Relativity, transformation of spin, existence of perfect or imperfect bodies and N-Central Systems etc. So, we define laws of physics observed by observer $O^{\prime}$ in central relativistic sense and observing microscopic and macroscopic laws differently and we have obtained these laws from one particular theory defined as "Universal Mechanics" which is valid in universal sense and particularly observed by observers at origin and at boundary of Universe in same manners. So, we have defined equations governing General Relativity at (macroscopic scale) or Quantum Mechanics (at microscopic scale) as-

$$
\begin{aligned}
& \Delta \rho_{\phi}=G_{x} E \\
& \text { (646) } \\
& E=\psi \phi=E_{p}+E_{c}=\psi_{p} \phi+\alpha \phi \Delta \phi \\
& \nabla^{2} \rho_{\phi}=G_{x}^{\prime} E \\
& \Delta s . \Delta F \geq c \hbar_{x} \\
& \Delta \Phi \Delta \psi \geq c \hbar_{x}\left(\frac{\Phi \Gamma(\Phi)}{(\psi+\alpha \Phi)}\right) \\
& i \hbar_{x} \frac{\partial \mathcal{P}(\tau, s)}{\partial \tau}=\left(E-\hat{\mathcal{Q}}_{\tau}\right) \cdot \mathcal{P}(\tau, s) \\
& \Rightarrow i \hbar_{x} \nabla_{\tau}^{\prime} \mathcal{P}(\tau, s)=E . \mathcal{P}(\tau, s) \\
& i \frac{\partial^{2} \mathcal{P}(\tau, s)}{\partial s^{2}}+\mathcal{P} . \mathcal{P}(\tau, s)=0
\end{aligned}
$$

$$
\left(i 2 \psi_{b} \frac{\partial}{\partial \tau}+\hbar_{x} \nabla^{2}\right) \mathcal{P}(\tau, s)=0
$$

$$
\left(i 2 \Delta \psi \frac{\partial}{\partial \tau}+\hbar_{x} \nabla^{2}\right) \mathcal{P}(\tau, s)=0
$$

$$
\left(2 \Delta \psi \nabla_{\tau}^{\prime}+\hbar_{x}\left(\nabla_{s}^{\prime}\right)^{2}\right) \mathcal{P}(\tau, s)=0
$$

$$
\left(2 \psi_{b} \nabla_{\tau}^{\prime}+\hbar_{x}\left(\nabla_{s}^{\prime}\right)^{2}\right) \mathcal{P}(\tau, s)=0
$$

$\Delta \phi \Delta \psi \geq \mathcal{T}_{h} \cdot \hbar_{x}$

$$
\dot{\mathcal{O}}=\frac{1}{\phi} \frac{\partial \phi}{\partial \tau}\{\mathcal{O}, E\}=\frac{1}{\psi^{\prime}} \frac{\partial \psi}{\partial \tau}\{\mathcal{O}, E\}
$$

$$
u\left(\phi_{b}, \psi_{b}\right)=\frac{8 \pi}{h_{x}^{2}}\left(\frac{\phi_{b} \psi_{b}}{\left(v_{p}\right)_{b}}\right)^{3} \frac{1}{e^{\theta_{b}}-1}
$$

$$
P_{x}=\sigma_{x} \mathrm{p}_{x}^{4} \frac{\left(\Delta F_{s}\right)^{4}}{\eta_{s}}\left(\frac{d \alpha_{s}}{d \Phi_{s}}\right)^{4}
$$

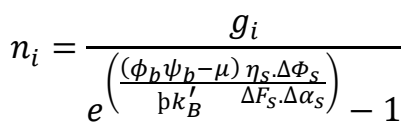

Or

$$
n_{i}=\frac{g_{i}}{e^{\left(\frac{(\phi \psi-\mu) \eta_{s} . \Delta \Phi_{S}}{\mathrm{p} k_{B}^{\prime} \Delta F_{s} \cdot \Delta \alpha_{S}}\right)}+1}
$$

$$
\left(v_{\phi}\right)_{\omega}=\sqrt{\frac{d P}{d \rho_{\phi}}}
$$

So, these all equations and the Generalized Principles and theorems like the Generalized Correspondence Principle, 
Generalized Complementary Principle, and Generalized Uncertainty Principle etc are obtained from same theory defined as "Universal Mechanics". So, Quantum Mechanics and General Relativity are unified in terms of Universal Mechanics which is more general in nature and in universal sense also. Generalized equivalence Principle also defined in my next paper on the unification of fundamental forces in terms of Universal Mechanics. So, this article unify both kinds of theories which were being unified by most recognized theoretical physicists of $20^{\text {th }}$ and $21^{\text {st }}$ century but they felt because of not understanding the "Principle of Central System Relativity". So, the determined task of this article is over as defined in abstract and introduction. So, I am going to give a break to this article by concluding some facts from it.

\section{CONCLUSIONS}

- The Quantum Mechanics and General Relativity both theories are two phases of a single theory "Universal Mechanics".

- Principle of Central System Relativity unifies both microscopic and Macroscopic Mechanics in Universal Mechanics.

- There exists a unique Principle of Central System Relativity which includes Galilean and Einstein's relativity over the universal mechanical terms also, defined by me as Principle of Universal Relativity.

- Einstein's special relativity only holds for some special cases and does not hold for complete $\phi-\psi$ transformation and General Relativity also does not specify the reason behind the curvature in spacetime but this is variation in the scalar field density around bodies.

- There exists Generalized Quantum Mechanics included in universal mechanic for all observers looking at $(\mathrm{k}-1)^{\text {th }}$ bodies from $\mathrm{k}^{\text {th }}$ bodies governing the Generalized Principles (like Generalized Correspondence Principle, Generalized Complementary Principle Generalized Uncertainty Principle etc).

- $\phi-\psi$ Transformation plays a vital role in the effects of special relativity like time dilation, length contraction and energy mass equivalence and Quantum Mechanics like Wave-Particle Duality, Uncertainty Principle etc or in other words the effects of both theories arise due to $\phi-\psi$ transformation.

- During critical epochs variation in conversion constant in zero.

- Bosons and fermions differ with each other because of their geometrical configuration in scalar fields is different. In this way Pauli Exclusion Principle and Spin Statistics Theorem both obtained in universal sense.

\section{ACKNOWLEDGEMENT}

I want to say thanks a lot to my friend Neeraj Meena for writing some of my equations digitally or very much love to my Grandfather Rameswar Lal and Father Heera Lal to support (financially or with all their blessings and trust) me in publishing this valuable paper in this Journal.

\section{APPENDIX}

APPENDIX A: SECOND DERIVATIVES OF GENERALIZED SCHRÖDINGER'S EQUATION WITH RESPECT TO SPACE AND TIME

Now differentiating again to equation (423) with respect to space parameter $s$ as-

$$
\begin{aligned}
\frac{\partial^{2}}{\partial s^{2}}\left(\frac{F . s . e^{i \theta}}{\hbar_{x}}\right)= & \frac{\partial}{\partial s}\left(\frac{F . s . e^{i \theta}}{\hbar_{x}}\right)\left[\left\{\frac{1}{F} \frac{\partial \psi}{\partial \tau}+\frac{1}{v_{p}} \frac{\partial v_{p}}{\partial s}+\frac{1}{s}\right.\right. \\
& \left.\left.-\frac{1}{\hbar_{x}} \frac{\partial \hbar_{x}}{\partial s}+i \frac{\partial \theta}{\partial s}\right\}\right]^{2} \\
& +\left(\frac{F . s . e^{i \theta}}{\hbar_{x}}\right)\left(-\frac{1}{F^{2}} \frac{\partial F}{\partial s} \frac{\partial \psi}{\partial \tau}+\frac{1}{F} \frac{\partial}{\partial s}\left(\frac{\partial \psi}{\partial \tau}\right)\right. \\
& -\frac{1}{v_{p}^{2}}\left(\frac{\partial v_{p}}{\partial s}\right)^{2}+\frac{1}{v_{p}} \frac{\partial^{2} v_{p}}{\partial s^{2}}-\frac{1}{s^{2}}+\frac{1}{\hbar_{x}^{2}}\left(\frac{\partial \hbar_{x}}{\partial s}\right)^{2} \\
& \left.-\frac{1}{\hbar_{x}} \frac{\partial^{2} \hbar_{x}}{\partial s^{2}}+i \frac{\partial^{2} \theta}{\partial s^{2}}\right)
\end{aligned}
$$

$$
\begin{aligned}
\frac{\partial^{2}}{\partial s^{2}}\left(\frac{F . s . e^{i \theta}}{\hbar_{x}}\right)= & \left(\frac{F . s . e^{i \theta}}{\hbar_{x}}\right)\left[\frac{1}{F} \frac{\partial \psi}{\partial \tau}+\frac{1}{v_{p}} \frac{\partial v_{p}}{\partial s}+\frac{1}{s}-\frac{1}{\hbar_{x}} \frac{\partial \hbar_{x}}{\partial s}\right. \\
& \left.+i \frac{\partial \theta}{\partial s}\right]^{2} \\
& +\left(\frac{F . s . e^{i \theta}}{\hbar_{x}}\right)\left(-\frac{1}{F^{2}} \frac{\partial F}{\partial s} \frac{\partial \psi}{\partial \tau}+\frac{1}{F} \frac{\partial}{\partial s}\left(\frac{\partial \psi}{\partial \tau}\right)\right. \\
& -\frac{1}{v_{p}^{2}}\left(\frac{\partial v_{p}}{\partial s}\right)^{2}+\frac{1}{v_{p}} \frac{\partial^{2} v_{p}}{\partial s^{2}}-\frac{1}{s^{2}}+\frac{1}{\hbar_{x}^{2}}\left(\frac{\partial \hbar_{x}}{\partial s}\right)^{2} \\
& \left.-\frac{1}{\hbar_{x}} \frac{\partial^{2} \hbar_{x}}{\partial s^{2}}+i \frac{\partial^{2} \theta}{\partial s^{2}}\right)
\end{aligned}
$$

$$
\begin{aligned}
\frac{\partial^{2}}{\partial s^{2}}\left(\frac{F . s . e^{i \theta}}{\hbar_{x}}\right)= & \left(\frac{F . s . e^{i \theta}}{\hbar_{x}}\right)\left[\left\{\frac{1}{F} \frac{\partial \psi}{\partial \tau}+\frac{1}{v_{p}} \frac{\partial v_{p}}{\partial s}+\frac{1}{s}-\frac{1}{\hbar_{x}} \frac{\partial \hbar_{x}}{\partial s}\right.\right. \\
& \left.+i \frac{\partial \theta}{\partial s}\right\}^{2} \\
& +\left\{-\frac{1}{F^{2}} \frac{\partial F}{\partial s} \frac{\partial \psi}{\partial \tau}+\frac{1}{F} \frac{\partial}{\partial s}\left(\frac{\partial \psi}{\partial \tau}\right)-\frac{1}{v_{p}^{2}}\left(\frac{\partial v_{p}}{\partial s}\right)^{2}\right. \\
& +\frac{1}{v_{p}} \frac{\partial^{2} v_{p}}{\partial s^{2}}-\frac{1}{s^{2}}+\frac{1}{\hbar_{x}^{2}}\left(\frac{\partial \hbar_{x}}{\partial s}\right)^{2}-\frac{1}{\hbar_{x}} \frac{\partial^{2} \hbar_{x}}{\partial s^{2}} \\
& \left.\left.+i \frac{\partial^{2} \theta}{\partial s^{2}}\right\}\right]
\end{aligned}
$$

In similar way differentiating equation for to two, with respect to space parameter- 


$$
\begin{aligned}
\frac{\partial^{2}}{\partial s^{2}}\left(\frac{E \cdot \tau \cdot e^{i \theta^{\prime \prime}}}{\hbar_{x}}\right)= & \left(\frac{E \cdot \tau \cdot e^{i \theta^{\prime \prime}}}{\hbar_{x}}\right)\left[\left\{\frac{1}{\psi} \frac{\partial \psi}{\partial s}+\frac{1}{\phi} \frac{\partial \phi}{\partial s}+\frac{1}{\tau \cdot v_{p}}\right.\right. \\
& \left.\left.-\left(\frac{E \cdot \tau}{\hbar_{x}}\right) \cdot \frac{\partial \hbar_{x}}{\partial \tau}+i E \cdot \tau \frac{\partial \theta^{\prime \prime}}{\partial \tau}\right\}^{2}\right] \\
& +\left(\frac{E \cdot \tau \cdot e^{i \theta^{\prime \prime}}}{\hbar_{x}}\right)\left(\frac{1}{\phi} \frac{\partial^{2} \phi}{\partial s^{2}}+\frac{1}{\psi} \frac{\partial^{2} \psi}{\partial s^{2}}\right. \\
& -\frac{1}{\phi^{2}}\left(\frac{\partial \phi}{\partial s}\right)^{2}-\frac{1}{\psi^{2}}\left(\frac{\partial \psi}{\partial s}\right)^{2} \\
& -\frac{1}{\tau v_{p}}\left(\frac{1}{\tau v_{p}}+\frac{1}{v_{p}} \frac{\partial v_{p}}{\partial s}\right)+\frac{1}{\hbar_{x}^{2}}\left(\frac{\partial \hbar_{x}}{\partial s}\right)^{2} \\
& \left.-\frac{1}{\hbar_{x}} \frac{\partial^{2} \hbar_{x}}{\partial s^{2}}+i \frac{\partial^{2} \theta^{\prime \prime}}{\partial s^{2}}\right)
\end{aligned}
$$

$$
\begin{aligned}
\frac{\partial^{2}}{\partial s^{2}}\left(\frac{E \cdot \tau \cdot e^{i \theta^{\prime \prime}}}{\hbar_{x}}\right)= & \left(\frac{E \cdot \tau \cdot e^{i \theta^{\prime \prime}}}{\hbar_{x}}\right)\left[\left\{\frac{1}{\psi} \frac{\partial \psi}{\partial s}+\frac{1}{\phi} \frac{\partial \phi}{\partial s}+\frac{1}{\tau \cdot v_{p}}\right.\right. \\
& \left.-\left(\frac{E \cdot \tau}{\hbar_{x}}\right) \cdot \frac{\partial \hbar_{x}}{\partial \tau}+i E \cdot \tau \frac{\partial \theta^{\prime \prime}}{\partial \tau}\right\}^{2} \\
& +\left(\frac{1}{\phi} \frac{\partial^{2} \phi}{\partial s^{2}}+\frac{1}{\psi} \frac{\partial^{2} \psi}{\partial s^{2}}-\frac{1}{\phi^{2}}\left(\frac{\partial \phi}{\partial s}\right)^{2}\right. \\
& -\frac{1}{\psi^{2}}\left(\frac{\partial \psi}{\partial s}\right)^{2}-\frac{1}{\tau v_{p}}\left(\frac{1}{\tau v_{p}}+\frac{1}{v_{p}} \frac{\partial v_{p}}{\partial s}\right) \\
& \left.\left.+\frac{1}{\hbar_{x}^{2}}\left(\frac{\partial \hbar_{x}}{\partial s}\right)^{2}-\frac{1}{\hbar_{x}} \frac{\partial^{2} \hbar_{x}}{\partial s^{2}}+i \frac{\partial^{2} \theta^{\prime \prime}}{\partial s^{2}}\right)\right]
\end{aligned}
$$

Now by putting some parameters for specification like it-

$$
\begin{gathered}
\mathfrak{B}=\left\{\frac{1}{F} \frac{\partial \psi}{\partial \tau}+\frac{1}{v_{p}} \frac{\partial v_{p}}{\partial s}+\frac{1}{s}-\frac{1}{\hbar_{x}} \frac{\partial \hbar_{x}}{\partial s}+i \frac{\partial \theta}{\partial s}\right\}, k_{1}=\left(\frac{F \cdot s \cdot e^{i \theta}}{\hbar_{x}}\right) \\
\mathfrak{B}^{\prime}=\frac{\partial \mathfrak{B}}{\partial s}=\left\{-\frac{1}{F^{2}} \frac{\partial F}{\partial s} \frac{\partial \psi}{\partial \tau}+\frac{1}{F} \frac{\partial}{\partial s}\left(\frac{\partial \psi}{\partial \tau}\right)-\frac{1}{v_{p}^{2}}\left(\frac{\partial v_{p}}{\partial s}\right)^{2}\right. \\
+\frac{1}{v_{p}} \frac{\partial^{2} v_{p}}{\partial s^{2}}-\frac{1}{s^{2}}+\frac{1}{\hbar_{x}^{2}}\left(\frac{\partial \hbar_{x}}{\partial s}\right)^{2}-\frac{1}{\hbar_{x}} \frac{\partial^{2} \hbar_{x}}{\partial s^{2}} \\
\left.+i \frac{\partial^{2} \theta}{\partial s^{2}}\right\} \\
\mathbb{D}=\left\{\begin{array}{c}
\left.\frac{1}{\psi} \frac{\partial \psi}{\partial s}+\frac{1}{\phi} \frac{\partial \phi}{\partial s}+\frac{1}{\tau \cdot v_{p}}-\left(\frac{E \cdot \tau}{\hbar_{x}}\right) \cdot \frac{\partial \hbar_{x}}{\partial \tau}+i E \cdot \tau \frac{\partial \theta^{\prime \prime}}{\partial \tau}\right\}, k_{2} \\
=\left(\frac{E \cdot \tau \cdot e^{i \theta^{\prime \prime}}}{\hbar_{x}}\right)
\end{array}\right.
\end{gathered}
$$

$$
\begin{aligned}
\mathfrak{D}^{\prime}=\left(\frac{1}{\phi} \frac{\partial^{2} \phi}{\partial s^{2}}+\right. & \frac{1}{\psi} \frac{\partial^{2} \psi}{\partial s^{2}}-\frac{1}{\phi^{2}}\left(\frac{\partial \phi}{\partial s}\right)^{2}-\frac{1}{\psi^{2}}\left(\frac{\partial \psi}{\partial s}\right)^{2} \\
& -\frac{1}{\tau v_{p}}\left(\frac{1}{\tau v_{p}}+\frac{1}{v_{p}} \frac{\partial v_{p}}{\partial s}\right)+\frac{1}{\hbar_{x}^{2}}\left(\frac{\partial \hbar_{x}}{\partial s}\right)^{2} \\
& \left.-\frac{1}{\hbar_{x}} \frac{\partial^{2} \hbar_{x}}{\partial s^{2}}+i \frac{\partial^{2} \theta^{\prime \prime}}{\partial s^{2}}\right)
\end{aligned}
$$

So, equation (424) becomes-

$$
\frac{\partial^{2} \mathcal{P}(\tau, s)}{\partial s^{2}}=i \mathcal{P}(\tau, s)\left[\left(k_{1}^{\prime \prime}-k_{2}^{\prime \prime}\right)+i\left(k_{1}^{\prime}-k_{2}^{\prime}\right)^{2}\right]
$$

By multiplying both sides with $i$, we get-

$$
i \frac{\partial^{2} \mathcal{P}(\tau, s)}{\partial s^{2}}=-\mathcal{P}(\tau, s)\left[\left(k_{1}^{\prime \prime}-k_{2}^{\prime \prime}\right)+i\left(k_{1}^{\prime}-k_{2}^{\prime}\right)^{2}\right]
$$

Now by putting whole part on right hand side as $P$, we get-

$$
i \frac{\partial^{2} \mathcal{P}(\tau, s)}{\partial s^{2}}+\mathcal{P} \mathcal{P}(\tau, s)=0
$$

Here-

$$
P=\left[\left(k_{1}^{\prime \prime}-k_{2}^{\prime \prime}\right)+i\left(k_{1}^{\prime}-k_{2}^{\prime}\right)^{2}\right]
$$

APPENDIX B: Solution of equation (424) 


$$
\begin{aligned}
& \frac{\partial^{2} \mathcal{P}(\tau, s)}{\partial s^{2}}=-\mathcal{P}(\tau, s)\left[( \frac { F . s . e ^ { i \theta } } { \hbar _ { x } } ) \left\{\frac{1}{F} \frac{\partial \psi}{\partial \tau}+\frac{1}{v_{p}} \frac{\partial v_{p}}{\partial s}+\frac{1}{s}\right.\right. \\
& \left.-\frac{1}{\hbar_{x}} \frac{\partial \hbar_{x}}{\partial s}+i \frac{\partial \theta}{\partial s}\right\} \\
& -\frac{E \cdot \tau \cdot e^{i \theta^{\prime \prime}}}{\hbar_{x}}\left\{\frac{1}{\psi} \frac{\partial \psi}{\partial s}+\frac{1}{\phi} \frac{\partial \phi}{\partial s}+\frac{1}{\tau \cdot v_{p}}\right. \\
& \left.\left.-\left(\frac{E . \tau}{\hbar_{x}}\right) \cdot \frac{\partial \hbar_{x}}{\partial \tau}+i E \cdot \tau \frac{\partial \theta^{\prime \prime}}{\partial \tau}\right\}\right]^{2} \\
& +i \mathcal{P}(\tau, s)\left[( \frac { F . s . e ^ { i \theta } } { \hbar _ { x } } ) \left\{\left(\frac{1}{F} \frac{\partial \psi}{\partial \tau}+\frac{1}{v_{p}} \frac{\partial v_{p}}{\partial s}\right.\right.\right. \\
& \left.+\frac{1}{s}-\frac{1}{\hbar_{x}} \frac{\partial \hbar_{x}}{\partial s}+i \frac{\partial \theta}{\partial s}\right)^{2} \\
& +\left(-\frac{1}{F^{2}} \frac{\partial F}{\partial s} \frac{\partial \psi}{\partial \tau}+\frac{1}{F} \frac{\partial}{\partial s}\left(\frac{\partial \psi}{\partial \tau}\right)-\frac{1}{v_{p}^{2}}\left(\frac{\partial v_{p}}{\partial s}\right)^{2}\right. \\
& +\frac{1}{v_{p}} \frac{\partial^{2} v_{p}}{\partial s^{2}}-\frac{1}{s^{2}}+\frac{1}{\hbar_{x}^{2}}\left(\frac{\partial \hbar_{x}}{\partial s}\right)^{2}-\frac{1}{\hbar_{x}} \frac{\partial^{2} \hbar_{x}}{\partial s^{2}} \\
& \left.\left.+i \frac{\partial^{2} \theta}{\partial s^{2}}\right)\right\} \\
& -\left(\frac{E \cdot \tau \cdot e^{i \theta^{\prime \prime}}}{\hbar_{x}}\right)\left\{\left(\frac{1}{\psi} \frac{\partial \psi}{\partial s}+\frac{1}{\phi} \frac{\partial \phi}{\partial s}+\frac{1}{\tau \cdot v_{p}}\right.\right. \\
& \left.-\left(\frac{E . \tau}{\hbar_{x}}\right) \cdot \frac{\partial \hbar_{x}}{\partial \tau}+i E \cdot \tau \frac{\partial \theta^{\prime \prime}}{\partial \tau}\right) \\
& +\left(\frac{1}{\phi} \frac{\partial^{2} \phi}{\partial s^{2}}+\frac{1}{\psi} \frac{\partial^{2} \psi}{\partial s^{2}}-\frac{1}{\phi^{2}}\left(\frac{\partial \phi}{\partial s}\right)^{2}\right. \\
& -\frac{1}{\psi^{2}}\left(\frac{\partial \psi}{\partial s}\right)^{2}-\frac{1}{\tau v_{p}}\left(\frac{1}{\tau v_{p}}+\frac{1}{v_{p}} \frac{\partial v_{p}}{\partial s}\right) \\
& \left.\left.\left.+\frac{1}{\hbar_{x}^{2}}\left(\frac{\partial \hbar_{x}}{\partial s}\right)^{2}-\frac{1}{\hbar_{x}} \frac{\partial^{2} \hbar_{x}}{\partial s^{2}}+i \frac{\partial^{2} \theta^{\prime \prime}}{\partial s^{2}}\right)\right\}\right]
\end{aligned}
$$

We can neglect second derivatives of $\hbar_{x}$ and $\theta^{\prime \prime}$ if these linearly depend on space parameter $s$.

Now if $F, E, \hbar_{x}$ are dependent on space $s$ and time $\tau$ parameters than-

$$
\begin{gathered}
i \hbar_{x} \frac{\partial \mathcal{P}(\tau, s)}{\partial \tau}=\left(E-\hat{\mathcal{Q}}_{\tau}\right) \cdot \mathcal{P}(\tau, s) \\
\hat{\mathcal{Q}}_{\tau}=-\left[\left(F \cdot v_{p}\right)+\left(\tau \frac{\partial E}{\partial \tau}-s \cdot \frac{\partial F}{\partial \tau}\right)+i \frac{\partial \theta^{\prime}}{\partial \tau}(E . \tau-F . s)\right. \\
\left.+\frac{1}{\hbar_{x}} \frac{\partial \hbar_{x}}{\partial \tau}(F . s-E . \tau)\right]
\end{gathered}
$$

$$
\widehat{E}^{\prime}=\widehat{E}-\hat{\mathcal{Q}}=i \hbar_{x} \frac{\partial}{\partial \tau}
$$

$$
i \hbar_{x} \frac{\partial \mathcal{P}(\tau, s)}{\partial s}=-\left(F+\hat{\mathcal{Q}}_{s}\right) \cdot \mathcal{P}(\tau, s)
$$

$$
\frac{\partial \mathcal{P}(\tau, s)}{\partial s}=i \mathcal{P}(\tau, s) \cdot \frac{\partial}{\partial s}\left[\left(\frac{F . s}{\hbar_{x}}\right)-\left(\frac{E . \tau}{\hbar_{x}}\right)\right]
$$

Now by including duality as we know-

$$
\begin{gathered}
\hat{\mathcal{Q}}_{s}=\left[s . \frac{\partial F}{\partial s}-\tau \frac{\partial E}{\partial s}+F-\frac{E}{v_{p}}+i F . s \frac{\partial \theta^{\prime}}{\partial s}-i E . \tau \frac{\partial \theta^{\prime \prime}}{\partial s}\right. \\
\left.-\frac{1}{\hbar_{x}} \frac{\partial \hbar_{x}}{\partial s}(F . s-E . \tau)\right]
\end{gathered}
$$

Or we get-

$$
\hat{F}^{\prime}=\hat{F}+\hat{\mathcal{Q}}_{s}=-i \hbar_{x} \frac{\partial}{\partial s}
$$

So, from here we get two operators as-

$$
\begin{array}{r}
\hat{F}=-i \hbar_{x} \frac{\partial}{\partial s}-\hat{\mathcal{Q}}_{s} \\
\hat{E}=i \hbar_{x} \frac{\partial}{\partial \tau}+\hat{\mathcal{Q}}_{\tau}
\end{array}
$$

Now as we know-

$$
E=E_{p}+E_{c}
$$

$$
\left(i \hbar_{x} \frac{\partial}{\partial \tau}+\hat{\mathcal{Q}}_{\tau}\right) \mathcal{P}(\tau, s)=\psi_{p} \phi+\frac{\left(-i \hbar_{x} \frac{\partial}{\partial s}-\hat{\mathcal{Q}}_{s}\right)^{2}}{2 \psi} \cdot \mathcal{P}(\tau, s)
$$

Or

$$
\left[2 \psi\left(i \hbar_{x} \frac{\partial}{\partial \tau}+\hat{\mathcal{Q}}_{\tau}\right)-\left(-i \hbar_{x} \frac{\partial}{\partial s}-\hat{\mathcal{Q}}_{s}\right)^{2}\right.
$$

$$
\left.-2 \psi_{p}\left(i \hbar_{x} \frac{\partial}{\partial \tau}+\hat{\mathcal{Q}}_{\tau}\right)\right] \mathcal{P}(\tau, s)=0
$$




$$
\left[2\left(\psi-\psi_{p}\right)\left(i \hbar_{x} \frac{\partial}{\partial \tau}+\hat{Q}_{\tau}\right)-\left(i \hbar_{x} \frac{\partial}{\partial s}+\hat{Q}_{s}\right)^{2}\right] \mathcal{P}(\tau, s)=0
$$

$$
\left(2 \Delta \psi \nabla_{\tau}^{\prime}+\hbar_{x}\left(\nabla_{s}^{\prime}\right)^{2}\right) \mathcal{P}(\tau, s)=0
$$

Here $\nabla_{\tau}^{\prime}=\left(i \hbar_{x} \frac{\partial}{\partial \tau}+\hat{Q}_{\tau}\right)$ and $\nabla_{s}^{\prime}=-\left(i \hbar_{x} \frac{\partial}{\partial s}+\hat{Q}_{s}\right)$ are extended energy and time Operators.

\section{REFERENCES}

[1] S. Mund, "On the Configuration of EM Waves and Bosons", IJRAMT, vol. 2, no. 7, pp. 162168, Jul. 2021.

[2] S. Mund, "Generalization of Different Type of Bodies Exist in Universe", IJRAMT, vol. 2, no. 7, pp. 213-220, Jul. 2021.

[3] S. Mund, "Formation and Stability of Various Type of Central Systems in Universe", IJRAMT, vol. 2, no. 7, pp. 293-301, Jul. 2021.

[4] S. Mund, "Generalization of All Fundamental Forces Exist in Universe", IJRAMT, vol. 2, no. 7, pp. 351-368, Aug. 2021.

[5] S. Mund, "Standard Definitions of All Fundamental Quantities Exist in Universe", IJRAMT, vol. 2, no. 8, pp. 122-143, Sep. 2021.

[6] S. Mund, "Generalization of Various Kinds of Scalar Fields Exist in Universe and Their Mathematical Representations", IJRPR, vol. 2, no. 11 , pp. 52-100, Nov. 2021.

[7] John Stachel and A. Einstein, "Einstein's Miraculous Year: five papers that changed the face of physics", Princeton University Press, 41 William Street, Princeton, New Jersey 08540, ISBN 0-691-05938-1 https://doi.org/10.2307/j.ctv1h9dh0m

[8] M. Planck, "On the Law of Energy Distribution in Normal Spectrum", Ann. Phys., vol. 4, no. 9, pp. 553-563, 7-January-1901.

[9] Acolyte Science. (2008). Der Lichtelektrische Effekt. http://www.acolytescience.co.uk/origins/herz.html.

[10] Bohr, N. "The quantum postulate and the recent development of atomic theory (Vol. 3)." Edinburgo: R. \& R. Clarke Ltd (1928).

[11] Bohr, N. "On the Constitution of Atoms and Molecules", Philosophical Magazine Series 6, Volume 26 July 1913, p. 1-2

[12] Einstein, A. The Field Equations of Gravitation. Sitzungsber.Preuss.Akad.Wiss.Berlin (Math.Phys.) 1915, 844-847 (1915).

[13] Louis de Broglie. Recherches sur la théorie des Quanta. Physique [physics]. Migration université en cours d'affectation, 1924. Français.
[14] Heisenberg,

W.

Schwankungserscheinungen

(1927)

quantenmechanik. Zeitschrift für Physik, 40(7), 501-506.

[15] Born, M. (1926). Quantenmechanik der stoßvorgänge. Zeitschrift für Physik, 38(11). 803-827.

[16] Born, M., Heisenberg, W., \& Jordan, P. (1926). Zur Quantenmechanik. II. Zeitschrift für Physik, 35(8), 557-615.

[17] Schrödinger, E. (1926). An undulatory theory of the mechanics of atoms and molecules. Physical review, 28(6), 1049.

[18] Jacobi, C. G. J. (1837). Ueber die Reduction der Integration der partiellen Differentialgleichungen erster Ordnung zwischen irgend einer Zahl Variablen auf die Integration eines einzigen Systemes gewöhnlicher Differentialgleichungen.

[19] Kragh, H. (1984). Equation with the many fathers. The Klein-Gordon equation in 1926. American Journal of Physics, 52(11). 1024-1033.

[20] Klein, O. (1926). Quantentheorie und fünfdimensionale Relativitätstheorie. Zeitschrift für Physik, 37(12), 895-906.

[21] Dirac, P. A. M. (1928). The quantum theory of the electron. Proceedings of the Royal Society of London. Series A, Containing Papers of a Mathematical and Physical Character, 117(778) 610-624.

[22] Galileo Galilei, "Two Chief World Systems Ptolemaic and Copernican", 2nd ed., translated by S. Drake (University of California Press, Berkeley, 1967), pp. 186-188.

[23] Mach, Ernst. The science of mechanics. Prabhat Prakashan, 1907.

[24] Edwin Hubble, "A relation between distance and radial velocity among extra-galactic nebulae", PNAS, vol. 3 , no. 15 , pp. 168-173, 15-March1929.

[25] C. Doppler, "On the colored light of the double stars and certain other stars of the heavens", Abh. Kniglich Bhmischen Ges. Wiss, 2, 467$482,1842$.

[26] Bohr, Niels. The penetration of atomic particles through matter. Copenhagen: Munksgaard, 1948.

[27] P.A. Cerenkov, "Visible Radiation Produced by electrons moving in a medium with velocities exceeding that of light", Phys. Rev., vol. 52, no. 4, pp. 378-379, August-1937.

[28] Eddington, Arthur Stanley. "The total eclipse of 1919 May 29 and the influence of gravitation on light." The Observatory 42 (1919): 119-122.

[29] Einstein, Albert. "Hamilton's principle and the general theory of relativity." Sitzungsber. Preuss. Akad. Wiss. Berlin (Math. Phys.) 1916 (1916): 1111-1116.

[30] Beurling, A. "FOR THE LAPLACE 
International Journal of Scientific Research in Engineering and Management (IJSREM)

EQUATION." Seminars on analytic functions.

Vol. 1. Institute for Advanced Study, 1958.

[31] Poisson (1823). "Mémoire sur la théorie du magnétisme en mouvement" [Memoir on the theory of magnetism in motion]. Mémoires de l'Académie Royale des Sciences de l'Institut de France (in French). 6: 441-570.

[32] Newton, Isaac. "Principia mathematica." Book III, Lemma V, Case 1 (1934): 1687.

[33] Bohr, Niels. Atomic theory and the description of nature. Vol. 1. CUP Archive, 1961.

[34] Widder, D.V. (1975), The heat equation, Pure and Applied Mathematics, 67, New YorkLondon: Academic Press [Harcourt Brace Jovanovich, Publishers]

[35] Michelson, A. A., F. G. Pease, and F. Pearson. "Repetition of the Michelson-Morley experiment." JOSA 18.3 (1929): 181_1-182.

[36] Born, Max, and J. Robert Oppenheimer. "On the quantum theory of molecules." Сборник статей к мультимедийному электронному учебнометодическому комплексу по дисциплине «физика атома и атомных явлений»/отв. ред. Шундалов МБ; БГУ, Физический факультет (1927).

[37] Lagrange, Joseph-Louis, "Mécanique Analytique”, United Kingdom, Cambridge University Press, 2009.

[38] Dirac, Paul Adrien Maurice. "Generalized hamiltonian dynamics." Canadian journal of mathematics 2 (1950): 129-148.

[39] Clausius, Rudolf. The mechanical theory of heat. Macmillan, 1879 .

[40] Boltzmann, Ludwig. "Ueber eine von Hrn. Bartoli entdeckte Beziehung der Wärmestrahlung zum zweiten Hauptsatze." Annalen der Physik 258.5 (1884): 31-39.

[41] Wien, Max. "Ueber die Berechnung und messung kleiner selbstpotentiale." Annalen der Physik 289.13 (1894): 928-947.

[42] Rayleigh, Lord. "LIII. Remarks upon the law of complete radiation." The London, Edinburgh, and Dublin Philosophical Magazine and Journal of Science 49.301 (1900): 539-540.

[43] Planck, Max. "On the law of distribution of energy in the normal spectrum." Annalen der physik 4.553 (1901): 1.

[44] Plastino, A. R., and A. Plastino. "Tsallis' entropy, Ehernfest theorem and information theory." Physics Letters A 177.3 (1993): 177179.

[45] Pauli, Wolfgang. "The connection between spin and statistics." Physical Review 58.8 (1940): 716.

[46] Fermi, Enrico. "Statistical method to determine some properties of atoms." Rend. Accad. Naz. Lincei 6.602-607 (1927): 5.

[47] Bose, Satyendra Nath. "Planck's law and the light quantum hypothesis." Journal of
Astrophysics and Astronomy 15 (1994): 3

[48] Preston, Frank W. "The canonical distribution of commonness and rarity: Part I." Ecology 43.2 (1962): 185-215.

[49] Pauli, Wolfgang. "Über den Zusammenhang des Abschlusses der Elektronengruppen im Atom mit der Komplexstruktur der Spektren." Zeitschrift für Physik 31.1 (1925) 765-783.

[50] "The Speed of Sound". mathpages.com. Retrieved 3 May 2015.

[51] Thorne, Kip S. "LIGO and gravitational waves, III: Nobel lecture, December 8, 2017." Annalen der Physik 531.1 (2019): Art-No.

\section{BIOGRAPHY}

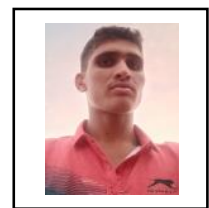

I am a Physicist and Cosmologist and Bachelors Student at Central University of Rajasthan in my final year, Here I enrolled as Integrated Master's Student of Physics. I entered here in 2018 just after completing my High School from PCM. I am Passionately Curious about Universe from my High School days and read many Theoretical Physics Books to explore it. I published 6 research papers in various international journals in theoretical physics and cosmology. My all articles are related with each other and indicate towards a new theory of cosmos which is applicable on whole universe unlike Quantum Mechanics and General Relativity. I this theoretical perspective I have unified all Fundamental Forces or Quantum Mechanics and General Relativity in terms of Central System Relativity. 